\title{
EVALUATING THE INOCULATION EFFECT \\ OF MUSIC ON STRESS REACTIVITY IN \\ HEALTHY YOUNG AND OLDER ADULTS:
}

THE MODERATING ROLE OF MUSIC

ABSORPTION

by

Katlyn J. Peck

Master of Arts, Ryerson University, 2013

Bachelor of Arts (Hons.), Brock University, 2011

\author{
A dissertation \\ presented to Ryerson University \\ in partial fulfillment of the \\ requirements for the degree of \\ Doctor of Philosophy
}

Toronto, Ontario, Canada, 2018

(C) Katlyn Peck, 2018 


\section{AUTHOR'S DECLARATION FOR ELECTRONIC SUBMISSION OF A DISSERTATION}

I hereby declare that I am the sole author of this dissertation. This is a true copy of the dissertation, including any required final revisions, as accepted by my examiners.

I authorize Ryerson University to lend this dissertation to other institutions or individuals for the purpose of scholarly research.

I further authorize Ryerson University to reproduce this dissertation by photocopying or by other means, in total, or in part, at the request of other institutions or individuals for the purpose of scholarly research.

I understand that my dissertation may be made electronically available to the public. 


\title{
Evaluating the inoculation effect of music on stress reactivity in healthy young and older adults: \\ the moderating role of music absorption
}

Doctor of Philosophy, 2018

\section{Katlyn Peck}

Psychology

Ryerson University

\begin{abstract}
Chronic exposure to stress at any age is associated with a myriad of negative physiological and psychological consequences, and as such, development of effective low-cost and non-invasive stress reduction interventions are important. Music listening has been shown to promote faster physiological recovery from acute stress. However, there is a paucity of research examining the potential inoculation effect of music on stress reactivity, as well as potential modifier's that may influence this effect such as music selection and music absorption. Hence, the current study examined the potential inoculation effect of music in response to acute stress, as measured by a comprehensive set of stress indices. It was hypothesized that listening to music prior to acute stress exposure would decrease stress reactivity compared to white noise (WN), and that selfselected music (SSM) would serve as a stronger inoculator than researcher-selected music (RSM). Finally, it was hypothesized that music absorption would moderate the inoculation effect of music, with a greater decrease in stress reactivity observed in high absorbers. Exploratory subgroups analyses were also performed to examine any potential age differences in the aforementioned associations. Participants were randomly assigned to either $\operatorname{RSM}(n=37)$, SSM $(n=38)$, or a WN group $(n=33)$ and listened to either music or white noise prior to undergoing
\end{abstract}


the Trier Social Stress Test (TSST). Outcome indices of stress included skin conductance, heart rate, salivary cortisol and self-report affect. Mixed analyses of covariance showed that music listening did not inoculate the stress response compared with WN and SSM did not serve as a more effective inoculator than RSM. A main effect of music absorption was found, suggesting that high absorbers are more reactive than low absorbers. Although the study hypotheses were not supported, exploratory sub-group analyses in older adults suggest that music listening and absorption may modulate the stress response. This study provides new insight into the effect of music listening on stress reactivity and presents a new line of questions that require further investigation. 


\section{Acknowledgements}

Words do not express the extent to which I can thank my supervisor, Dr. Alexandra Fiocco for not only believing in my project, but also believing in me as a graduate student and young investigator. She has spent countless hours guiding me and supporting my research interests, and focusing on my development as a student, and as a researcher. I will be forever grateful for the knowledge and opportunities she has provided me, and I can only hope that I will make her proud in the future. I have developed so much as a researcher, and as a person under her guidance and supervision. She strives to make every one of her students better in every way, and I feel that I have benefitted enormously being a part of that, both personally and professionally.

This dissertation was funded partly by the Harry Rosen Research Grant. I am endlessly grateful for his contribution to the Psychological Science program at Ryerson. Harry Rosen's considerate donation has enabled me to purchase supplies necessary to carry out my research, and for that, I am eternally grateful.

I would like to acknowledge Dr. Frank Russo, not only as a member of my supervisory committee, but also a supportive mentor, who has provided invaluable feedback with regards to my study. I would also like to thank members of my examination committee, Dr. Kristin Vickers, Dr. Souraya Sidani, and Dr. Jessica Grahn, for taking the time to read my dissertation with a critical eye.

I would also like to thank Shruti Vyas, Roxanne Choy, and Alexandra Giordano for their invaluable contributions to this dissertation study. Without your help, this study would not have been possible, and I am so grateful for the time and effort you both spent helping me at various stages of this study, from participant recruitment, to data processing and analyses. 
Undeniably, I have grown so much as a researcher, and as a person being surrounded by my Stress \& Healthy Aging Research (StAR) lab family. I am forever grateful for Vivian Huang, Sasha Mallya, and Laura Krieger for the support you have given me over the past few years. I am so grateful for the relationships that we have cultivated, and I have greatly benefitted from being in your presence during this process. You have all been an unconditional source of support and friendship throughout my time as a graduate student, and I am so grateful to have such supportive, and compassionate peers to share my experience with.

Of course, this would not be complete without acknowledging my main source of support through this journey, my father, Bruce Peck. Dad, you have been a constant source of support and have always believed in me as a person, and as a student. There is no doubt that I would not have been able to complete this program without you. And for that, I will always be thankful. 


\section{Dedication}

I dedicate this dissertation and the journey that has accompanied it to my father.

I also dedicate this dissertation to my mother. Mom, you always provided me with a constant source of support, and the belief that I was worthy to succeed in anything I put my mind to.

Although you are no longer with me, I know that wherever you are, you are proud of me for the person I have become throughout this process. 


\section{Table of Contents}

Author's Declaration For Electronic Submission Of A Dissertation...................................................................ii

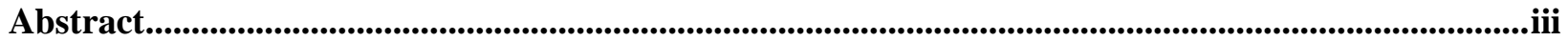

Acknowledgements ....................................................................................................................................................... v

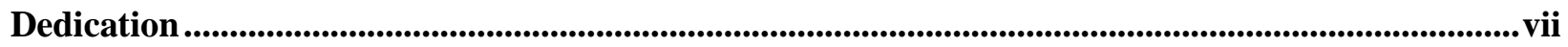

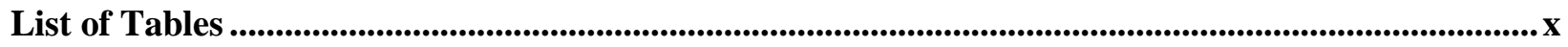

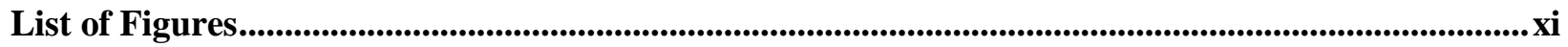

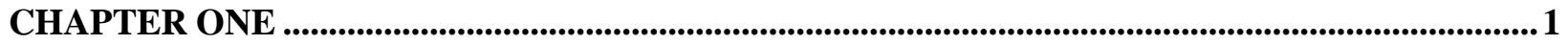

1. Background and Identification of the Problem ............................................................................... 1

CHAPTER TWO: Stress and Stress Physiology .........................................................................................3

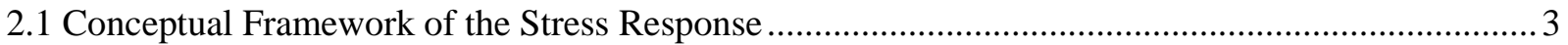

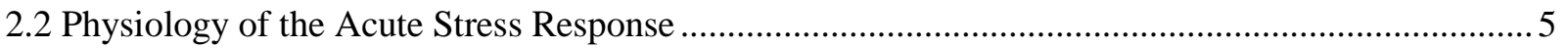

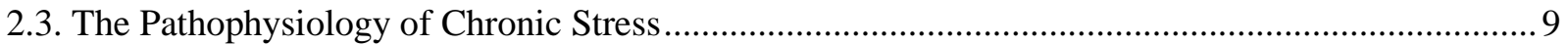

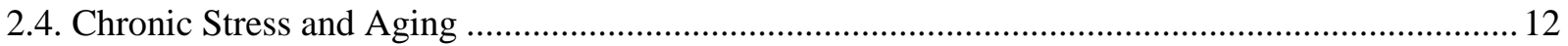

CHAPTER THREE: Stress Induction and Measurement .........................................................................16

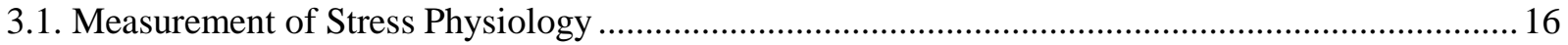

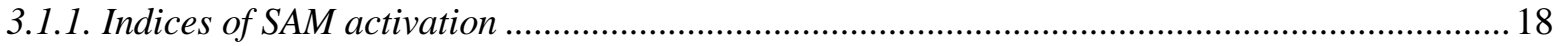

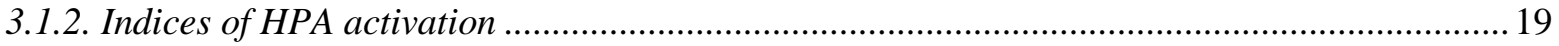

CHAPTER FOUR: Music and Well-Being ................................................................................................22

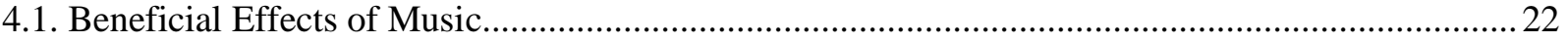

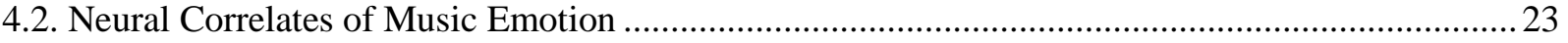

4.3. Psychophysiology of Music and Stress Reactivity ......................................................................24

4.4. Individual Differences in Music Selection and Music Absorption ................................................26

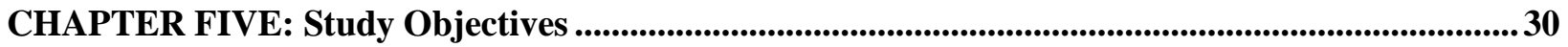

5.1. Gaps in the Current Literature and Study Objectives ................................................................... 30

5.2. Research Aims and Hypotheses .............................................................................................. 31

CHAPTER SIX: Method ....................................................................................................................................33

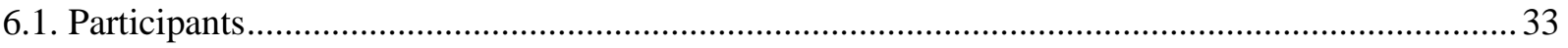

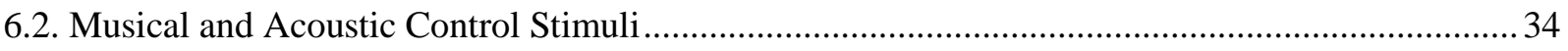

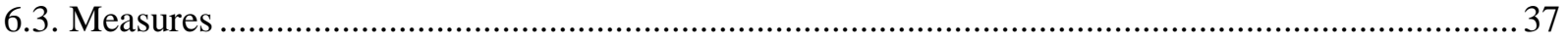

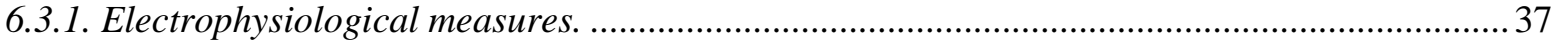

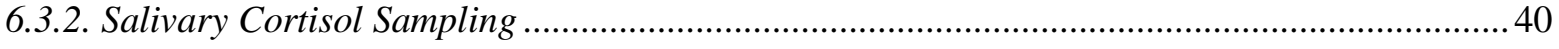

6.3.3. Psychological questionnaires. …….................................................................................. 43

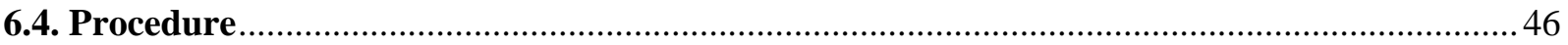




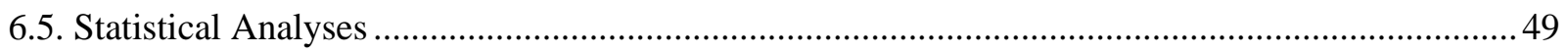

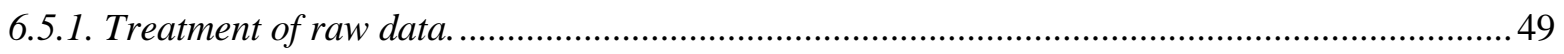

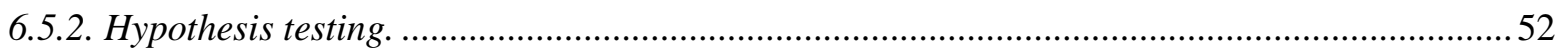

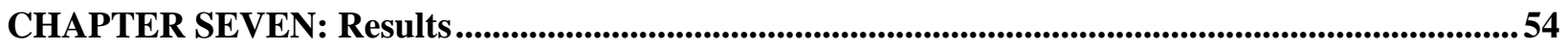

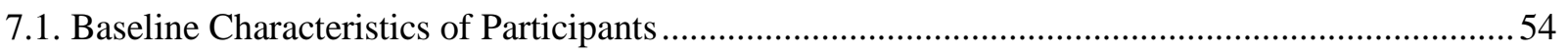

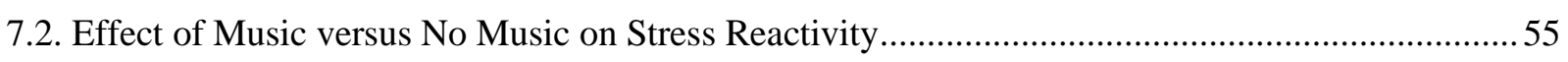

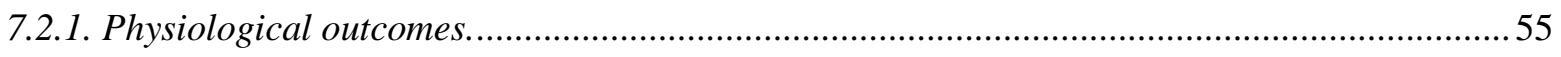

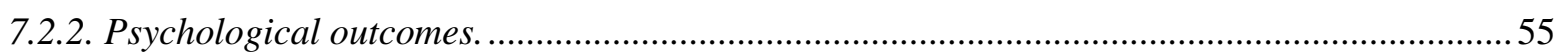

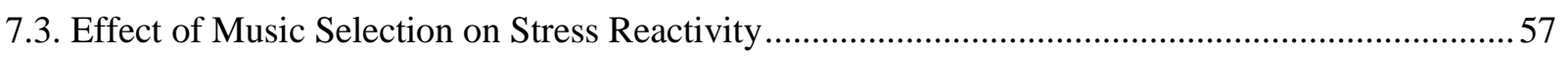

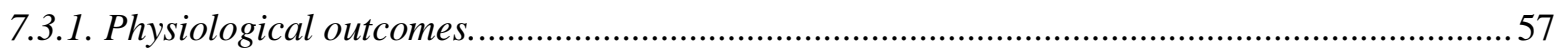

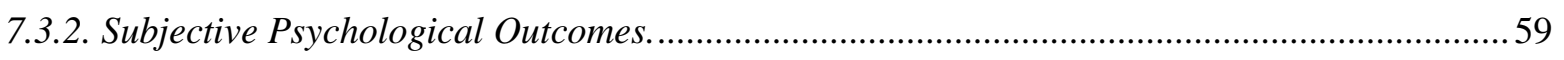

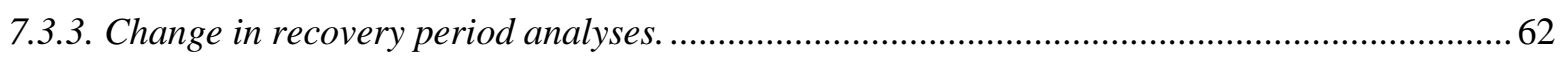

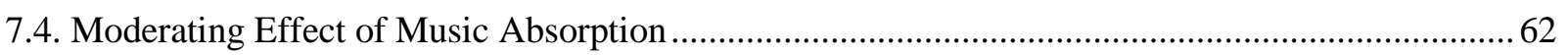

7.4.1. Effect Modification of Music Absorption on Physiological Outcomes. .................................... 62

7.4.2. Effect Modification of Music Absorption on Psychological Outcomes..................................... 65

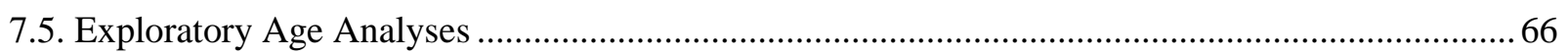

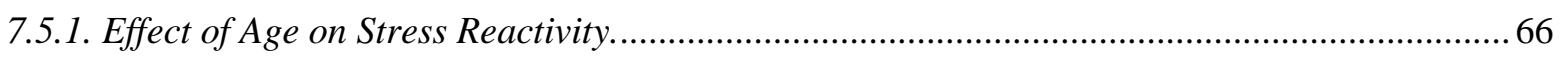

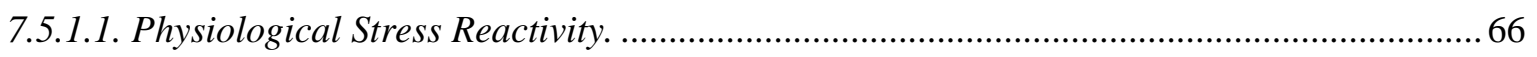

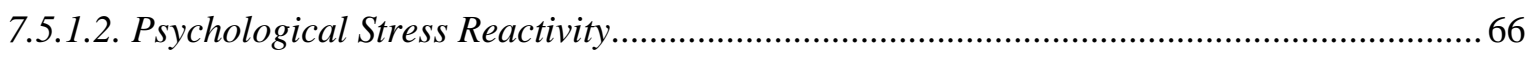

7.5.2. Effect of Music on Physiological Outcomes in Young and Older Adults.................................66

7.5.3. Effect of Music on Subjective Psychological Outcomes in Young and Older adults. ............... 68

7.5.4. Effect of music on change in recovery period in young and older adults...............................69

7.5.5. Age differences in music absorption as an effect modifier..................................................... 71

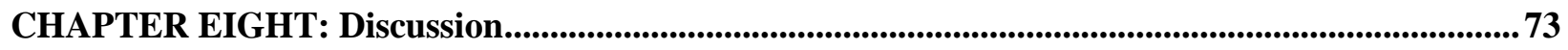

8.1 Music Listening as a Technique to Inoculate Future Stress Reactivity ........................................ 74

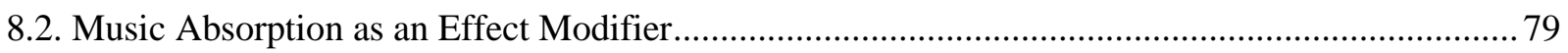

8.3. Age Differences in the Inoculation Effect of Music ................................................................. 81

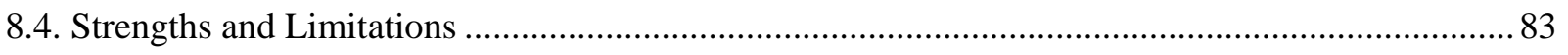

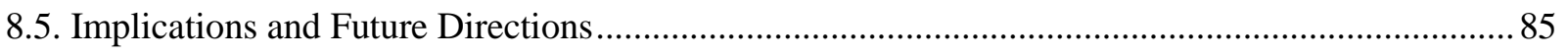

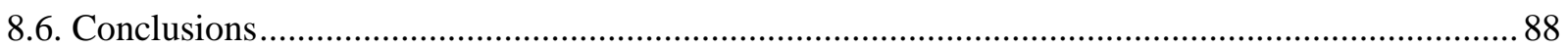

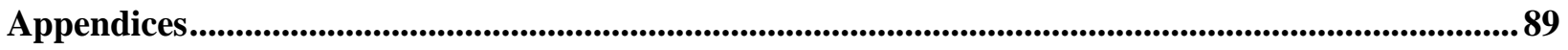

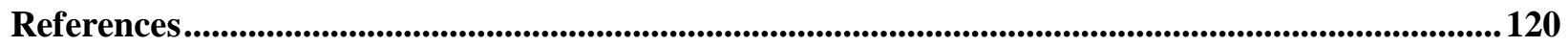




\section{List of Tables}

Table 1: Pilot study $(n=17)$ on arousal and valence of researcher-selected music ........................................36

Table 2: Baseline characteristics of sample by condition .................................................................................54

Table 3: Mixed analysis of covariance results: Effect of music exposure (vs. white noise) on

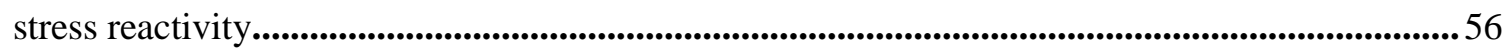

Table 4: Mixed analysis of covariance results: Testing the effect of music selection on stress reactivity .61 


\section{List of Figures}

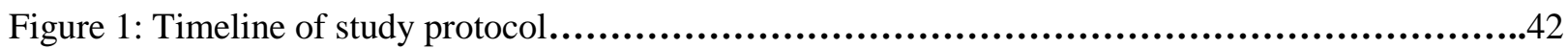

Figure 2a: Mean (SE) Skin Conductance Level (SCL) across time by Condition ........................57

Figure 2b: Mean (SE) Skin Conductance Response (SCR) count across time by Condition...............58

Figure 2c: Mean (SE) Heart Rate across time by condition..........................................58

Figure 2d: Mean (SE) Heart Rate Variability (HRV) across time by condition...........................58

Figure 2e: Mean (SE) salivary cortisol across time by condition.....................................58

Figure 2f: Mean subjective distress across time by condition......................................60

Figure 2g: Mean subjective excitement across time by condition.......................................60

Figure 2h: Mean subjective discomfort across time by condition....................................60

Figure 2i: Mean subjective agitation across time by condition......................................60

Figure 3a: Mean HRV in the WN condition in high and low absorbers across time.............................................................64

Figure 3b: Mean HRV in the RSM condition in high and low absorbers across time.

Figure 3c: Mean HRV in the SSM condition in high and low absorbers across time.

Figure 4a: Mean excitement ratings across time in high and low absorbers. Mean ratings square root (SQRT) transformed ...............................................................65

Figure 4b: Mean agitation ratings across time in high and low absorbers. Mean ratings SQRT transformed.......................................................................65

Figure 5: Mean SCL in (a) older adults and (b) young adults following the TSST (T4) and during recovery (T6-T8). Mean SCL scores are square-root transformed...........................67 


\section{CHAPTER ONE}

\section{Background and Identification of the Problem}

According to Statistics Canada (Statistics Canada, 2013), over 1 in 4 Canadians report daily stress levels in the high to severe range. Chronic perceived stress is an insidious phenomenon associated with adverse health outcomes (McEwen, 2008). Although acute reactivity to perceived stress is adaptive in the short run, chronic or intermittent activation of stress-sensitive systems, namely the sympathetic-adrenal-medulla (SAM) axis and the hypothalamic-pituitary-adrenal (HPA) axis, is associated with increased risk for cardiovascular disease, impaired cognitive function, depression, chronic fatigue, accelerated aging and early mortality (Chrousos, 2009). Older individuals are particularly vulnerable to chronic activation of stress-sensitive systems due to normal age-related changes in neuroendocrine and metabolic function (Lupien, McEwen, Gunnar, \& Heim, 2009). Consequently, the development of low-cost strategies that minimize the effect of stress on the individual is paramount.

Music is an art form that is documented to reduce levels of stress through the modulation of physiological arousal (Chanda \& Levitin, 2013) and is further shown to significantly reduce perceptions of anxiety (Sendelbach et al., 2006). Although the literature is somewhat mixed, recent research shows that compared to white noise, exposure to calming music following an acute stressor can facilitate faster recovery from stress, evidenced by rapid decline in skin conductance level (SCL; Sandstrom \& Russo, 2010; Sokhadze, 2007) and attenuation of cortisol, the primary stress hormone in humans (Khalfa et al., 2003). Although previous studies suggest that music may facilitate recovery following stress exposure, there is a paucity of research evaluating the potential inoculation effect of music when presented before the onset of a stressor. Indeed, programs designed to target minimizing stress reactivity, such as Stress Inoculation 
Training (SIT; Meichenbaum, 1972; Meichenbaum \& Turk, 1976) and Mindfulness-BasedStress Reduction (MBSR; Kabat-Zinn, 1982, 1990), have shown reductions in perceived stress (Beddoe \& Murphy, 2004; Grossman, Niemann, Schmidt, \& Wallach; Smith et al., 2008). Further, given mixed findings in the current literature, it may be suggested that individual differences, such as music responsiveness, moderate the inoculation effect. Indeed, faster recovery from an acute stressor while listening to music is reportedly greater in participants who report being "absorbed", or having a heightened tendency to be emotionally affected by music (Sandstrom \& Russo, 2013). Another possible reason for mixed findings is music selection; much of the literature focuses on experimenter-selected music in the context of stress reduction. The goal of the proposed study is to evaluate the inoculation effect of music on subsequent psychological and physiological stress-reactivity and to assess the moderating role of music absorption in both younger and older adults.

To provide footing for the study rationale and design, Chapter 2 of this dissertation will begin with an overview of the prominent stress theories and a description of stress physiology and its systems in the context of acute and chronic stress. Chapter 3 will provide a brief background on measurement tools used to examine stress physiology, with a focus on those that are relevant to this dissertation. Chapter 4 will then present a comprehensive review on the neuroscience of music and previous research that examines the effect of music on stress physiology. Finally, gaps in the current literature will be presented in Chapter 5, followed by the research rationale, objectives, and hypotheses of the study. Together, these chapters provide the necessary foundation for the current dissertation study. 


\section{CHAPTER TWO \\ Stress and Stress Physiology}

\subsection{Conceptual Framework of the Stress Response}

Homeostasis is a complex balance or state of equilibrium among varying physiological systems and parameters, which every living organism must maintain for survival (Cannon, 1929; Chrousos \& Gold, 1992). Walter Cannon was the first to describe the potential for external and internal stimuli to perturb this internal milieu, causing disequilibrium in set physiological values by increasing nervous and hormonal systems. Through his animal research, Cannon coined the Fight-or-Flight response, which is a reaction evoked by the organism in response to an acute noxious stimulus (Cannon, 1914; Henry, 1992). In particular, Walter Cannon reported on the role of the autonomic nervous system, and more specifically, the role of epinephrine and its widespread effects on the body in facilitating the fight-or-flight response.

The term "stress" was originally coined by Hans Selye, who defined stress as a "nonspecific response pattern of the body to any demand that is placed upon it" (Selye, 1936; 1976). According to Selye, regardless of the type of noxious stimulus (e.g., extreme heat, extreme cold etc.), the organism will respond with the same physiological pattern. Selye proposed the General Adaptation Syndrome (GAS) model in order to explain this non-specific physiological response of the organism if maintained beyond the "acute" response, becoming chronic in nature. These three stages included the alarm reaction, resistance and exhaustion stage.

Given that our environment is not static in nature, the ability of the organism to maintain homeostasis and be able to constantly adapt within a changing environment is referred to as “Allostasis" (Sterling \& Eyer, 1988). Homeostasis involves the coordinated regulation of numerous physiological systems that work to maintain a relatively stable internal equilibrium, whereas allostasis allows the organism to change its internal milieu to adapt to environmental 
demands accordingly (Juster, McEwen, \& Lupien, 2010; Sterling \& Eyer, 1988). Hence, homeostasis is concerned with the maintenance of internal static biological set-points (i.e., body temperature), allowing for the optimal functioning of physiological systems, whereas allostasis is concerned with dynamic biological set-points that allow the body to adapt to a changing environment (Juster et al., 2010; Shulkin, 2003). Allostasis is considered to be a modern conception of Selye's resistance stage, where adapting to the stressor is protective (McEwen, 2005). While allostasis is an adaptive and necessary response, chronic or frequent intermittent stress exposure can result in chronic activation of stress sensitive systems, namely the HPA and SAM axes, leading to wear and tear of the system, termed "allostatic load" (AL; McEwen \& Stellar, 1993), considered to be analogous to Selye's final exhaustion stage (McEwen, 2005).

The perception and experience of internal and external stressors can threaten or compromise this fine balance among interconnected physiological systems. Stress can arise when "individuals perceive that they cannot adequately cope with the demands being made on them or with threats to their well-being" (Lazarus, 1966). A stressor then, is something that actually threatens, or is perceived to threaten the disruption of the homeostatic balance within an individual (Chrousos, 2009), and is commonly characterized by the experience of high arousal and negative affect (Van Eck, Nicolson, \& Berkhof, 1998). Stressors can be psychological (e.g., worry or anxiety of a real or perceived threat), physical (e.g., physical trauma), environmental (e.g., experience of a natural disaster), or immunological (e.g., infectious disease) in nature, and can vary in duration and frequency (Klein \& Corwin, 2008).

More modern conceptions of stress suggest that the stress response has a degree of specificity, which is dependent on the type of threat to homeostasis (Goldstein \& McEwen, 2002). Unlike Selye's model, the individual's perception, appraisal, and anticipation of the 
stressor itself, and their available resources to cope with it (Goldstein, 2001; Goldstein \& Kopin, 2007) are emphasized as important factors involved in mounting a stress response (Folkman \& Lazarus, 1985). Further, research suggests that uncontrollability, unpredictability, novelty, and social-evaluative threat (i.e., performance is judged negatively by others) are situational determinants of a stressful experience (Dickerson \& Kemeny, 2004; Mason, 1968). More recently, Lupien and colleagues (2013) presented a theoretical model incorporating similar elements of novelty $(\mathrm{N})$, uncontrollability $(\mathrm{U})$, threat to one's ego (T; intended to represent social-evaluative threat), and low sense of control (S), using the acronym "NUTS" to help to recognize and conceptualize the factors involved in the stress response. The presence of one or more of the determinants outlined in the NUTS model is capable of eliciting a stress response.

\subsection{Physiology of the Acute Stress Response}

Both cortisol and catecholamines, the end products of our stress-sensitive systems, are secreted and released throughout the day under basal (non-stressed) conditions, following a circadian rhythm (Sapolsky, Krey, \& McEwen, 1986; Tsigos \& Chrousos, 2002). The diurnal pattern of cortisol has been closely examined, characterized by a rise in cortisol upon awakening within the first 30 minutes (Kaltas \& Chrousos, 2007), known as the cortisol awakening response (CAR; Pruessner et al., 1997), followed by a continuous decrease throughout the day, with the lowest levels documented in the evening (Baum \& Grunberg, 1995; Kudielka et al., 2012; Lovallo \& Thomas, 2000; Nader, Chrousos, \& Kino, 2010). Some studies, however, document individual variations in the diurnal pattern with some participants displaying more flattened, or inconsistent diurnal cycles (Stone et al., 2001; Fiocco et al., 2006). Within the stress literature, the diurnal cycle of cortisol is pertinent when considering the effect of stress exposure on hormone secretion, with more profound increases in cortisol observed in the afternoon compared 
with morning reactive levels (Kudielka \& Wust, 2010).

The brain is the central organ that determines what is stressful, and the evaluation of a stressor is largely dependent on activation of the hippocampus (involved with learning and memory processes) and the amygdala (a key brain region involving the regulation of fear and anxiety; Chrousos, 2009). The amygdala and hippocampus are integral structures involved in the stress response. Activation of the amygdala innervates the paraventricular nucleus (PVN) of the hypothalamus (Herman et al., 2005) following the perception of a stressor, whereas the hippocampus exerts inhibitory effects on the PVN, helping to regulate the termination of the stress response (Ulrich-Lai \& Herman, 2009).

Stimulation of the PVN triggers a cascade of events in both the SAM and HPA systems in response to stress. The sympathetic nervous system (SNS), specifically the SAM system, is activated which initiates a short-term, "fight-or-flight" response to stress (Gunnar \& Quevedo, 2007), which is akin to Selye's alarm reaction. Activation of the SAM system prompts release of catecholamines norepinephrine $(\mathrm{NE})$ and epinephrine $(\mathrm{E})$ from the adrenal medulla into the bloodstream (Kemeny, 2003; Piazza, Almeida, Dmitrieva, \& Klein, 2010; Tsigos \& Chrousos, 2002). Increased circulating NE and E are metabolized within minutes (Piazza et al., 2010), and are accompanied by an increase in autonomic functioning including, but not limited to, increased heart rate, blood pressure, respiration, and skin conductance (Gold \& Chrousos, 2002; Jansen et al., 1995; Uchino, Cacioppo, Malarkey \& Glaser, 1995). This increase in autonomic activity facilitates and directs attention and arousal to the immediate threat while inhibiting functions that are less adaptive in the current situation, such as digestion and sexual behavior (Gold, Goodwin, \& Chrousos, 1988; Henry, 1992).

Activation of the amygdala, leads to subsequent activation of the locus coeruleus (LC), a 
nucleus in the pons that releases NE. Evidence from rat models demonstrate that activation of the LC-NE pathway (Kaltas \& Chrousos, 2007) in response to acute stress (i.e., immobilization stress) results in the release of NE in stress-related brain regions including the amygdala and the medial prefrontal cortex (mPFC; Morilak et al., 2005) and serves to regulate the SAM response (Gunnar \& Quevedo, 2007). Fear-related behaviours generated by the amygdala are crucial for survival, as they enable the organism to react rapidly to defend and protect against harm when experiencing a threatening situation.

In parallel to the rapid acting SAM axis (Piazza et al., 2010) is activation of the HPA axis, which facilitates the fight-or-flight response and presents a more prolonged activation that can be observed within a range of approximately 10-20 minutes following stress exposure (Allen et al., 2014; Kemeny, 2003; Kirschbaum \& Hellhammer, 1989). The PVN of the hypothalamus is activated when a stressor is perceived, triggering the release of corticotropin-releasing hormone (CRH; Lupien et al., 2009; Tsigos \& Chrousos, 2002). This in turn stimulates the anterior pituitary to release adrenocorticotropin hormone (ACTH) and arginine vasopressin (AVP). ACTH subsequently stimulates the adrenal cortex to release glucocorticoids (GCs), or cortisol in humans (Klein \& Corwin, 2007; Lupien et al., 2009). Secretion and release of cortisol into the blood portal results in secondary physiological changes including changes in immune and inflammatory function, increased vigilance and cardiovascular output, and increased glucogenesis (Herman \& Cullinan, 1997; Tsigos \& Chrousos, 2002), all of which may provide the organism (or the individual) with the necessary physiological resources required to adapt to environmental demands. Tertiary outcomes such as cardiovascular disease and diabetes can manifest if the system becomes overly taxed and dysregulated (Juster et al., 2011).

Cortisol also crosses the blood brain barrier, binding to glucocorticoid receptors (GR's) 
in the brain, namely in the hippocampus and prefrontal cortex (De Kloet, 1991; Sapolsky et al., 2000). Once the stressor subsides, cortisol plays a key role in regulating the stress response by providing inhibitory feedback at various levels of the HPA axis and at the level of the brain (Lupien et al. 2009a; Tsigos \& Chrousos, 2002). For instance, this negative feedback loop attenuates the release of $\mathrm{CRH}$ from the hypothalamus, inhibiting subsequent release of ACTH from the anterior pituitary (Herman et al., 2005; Johnson, Kamilaris, Chrousos, \& Gold,1992; Piazza et al., 2010). Further, cortisol also regulates the stress response by binding to glucocorticoid receptors at the level of the pituitary and adrenal gland, attenuating further release of ACTH and cortisol respectively (Lupien et al., 2009). Finally, cortisol binds to the hippocampus, further facilitating inhibition of the HPA system. Indeed, animal (Dunn \& Orr, 1984) and human studies (Rubin, Mandell, \& Crandall, 1966) demonstrate that when the hippocampus is stimulated, a decrease in glucocorticoid secretion ensues. This complex feedback loop is designed to protect the organism against excessive cortisol production, facilitating allostasis of the organism.

Taken together, both the SAM and HPA axes are complex interconnected systems that interact with multiple brain structures and pathways. Perception of a stressor results in the activation of numerous coordinated and interconnected systems, including the autonomic (sympathetic and parasympathetic systems), neuroendocrine, metabolic, and immune systems (Axelrod \& Reisine, 1984; Glaser \& Kiecolt-Glaser, 2005; Lupien, et al., 2009). Perturbation of these systems in response to an acute stressor results in various physiological changes that are necessary for adaptation and survival of the organism in the short term (Gold \& Chrousos, 2002; Lupien, et al., 2009; Piazza et al., 2010). 


\subsection{The Pathophysiology of Chronic Stress}

Although adaptive in the short run, prolonged or chronic activation of the SAM and HPA systems may lead to dysregulation of feedback mechanisms and other interconnected physiological systems, resulting in AL (Lupien et al., 2009). AL is a cumulative measure of physiological dysregulation and is associated with an imbalance across multiple regulatory systems resulting in considerable health risks (McEwen \& Stellar, 1993).

Indeed, chronic exposure to stress can have physiological consequences, as indicated by dysregulation of the SAM axis (Curtis, \& O'Keefe, 2002; Grippo et al., 2002; Krantz \& McCeney, 2002; Remus et al., 2015; Rozanski, et al., 1999). Animal research demonstrates that exposure to chronic mild stress results in an elevated resting heart rate, decreased heart rate variability (HRV), and exaggerated reactivity when experiencing acute environmental stressors compared with control rodents (Grippo et al., 2002; Grippo et al., 2006). In humans, decreased HRV is typically observed in individuals experiencing chronic psychological stress (Chandola et al., 2008), and is a robust predictor of mortality in both high and low-risk populations (Camm et al., 2004; Kleiger, Miller, Bigger, \& Moss, 1987; Thayer, Yamamoto, \& Brosschot, 2010; Tsuji et al., 1994). Additional secondary outcomes such as elevated resting heart rate, and elevated levels of NE (Carney et al., 1999; Carney, Freedland, \& Veith, 2005; Lake, et al., 1982) are associated with chronic activation of the SAM system, whereas lower HR reactivity in response to psychological stress is associated with hypoactivity of the SAM system (Brindle, Ginty, and Conklin, 2013; Phillips, Hunt, Der, \& Carroll, 2011). Indeed, alterations in cardiovascular function as a result of chronic and repeated activation of the SAM system can ultimately lead to dysregulation of the cardiovascular system, contributing to the development of hypertension (Markovitz et al., 2004; Guimont et al., 2006). 
Chronic exposure to glucocorticoids can lead to dysregulation of the HPA system. Some studies document increased basal activity of the HPA system evidenced by higher salivary cortisol levels (Ockenfels et al., 1995), whereas some studies document decreased HPA activity (Caplan, Cobb, \& French, 1979). Exposure to either chronically high or low levels of glucocorticoids is associated with pathology (Gold \& Chrousos, 2002; Raison \& Miller, 2003), while more acute, or moderate increases are associated with psychological health (Kemeny, 2003; Lupien et al., 2009). For instance, hyper-activity of the HPA axis resulting from chronic stress exposure is associated with excessive glucocorticoid secretion (i.e., hypercortesolemia), increased responsivity to future stressors (Sharpley, 2009; Ulrich-Lai \& Herman, 2009), and increased cortisol secretion throughout the day relative to unstressed individuals (Schulz et al., 1998).

Some studies also report hypoactivity of the HPA system (Heim, Ehlert, \& Hellhammer, 2000; Miller, Cohen, \& Ritchey, 2002) and glucocorticoid deficiency (i.e., hypocortisolemia), which can have detrimental effects on the brain and body. Hypocortisolemia is thought to occur following prolonged periods of HPA axis hyperactivity resulting, but not limited to reduced synthesis or release of $\mathrm{CRH}, \mathrm{ACTH}$, or cortisol, subsequently reducing the amount of circulating cortisol, and decreased effects of cortisol on target receptors (Fries, Hesse, Hellhammer, \& Hellhammer, 2005; Sharpley, 2009). Indeed, some studies show that individuals exposed to chronic stress present with hyposecrection of ACTH and cortisol under basal conditions (Yehuda et al., 1993).

Given that cortisol plays a key role in regulation of glucose storage and utilization, and inflammatory responses, the metabolic and immune systems can also become dysregulated with chronic exposure to stress. Cortisol can interfere with the action of insulin by inhibiting insulin 
secretion from the pancreas (Lambillotte et al., 1997), which can lead to insulin resistance. Additionally, low concentrations of high-density lipoproteins (HDL), or "good cholesterol" and glucose intolerance have been documented in those with altered cortisol secretion (Andrews et al., 2002; Phillips et al., 1998). Furthermore, both diminished immune responses have been documented in chronically stressed individuals resulting in heightened incidence of infection (Anderson, Kiecolt-Glaser, \& Glaser, 1994; Cohen \& Williamson, 1991), as well as excessive immune function (allergic reactions; Segerstrom \& Miller, 2004). Chronic stress can result in glucocortiocoid receptor resistance (GCR), which in turn can create an inability to downregulate inflammatory response, as documented by an increased production of proinflammatory cytokines (Cohen et al., 2012). Inflammation plays an important role in the development and progression of disease, chronically stressed individuals can be at a heightened risk for development of a wide-range of stress-related disorders.

Chronic activation of the SAM axis can lead to damage of the vasculature, resulting in increased risk for pathology (Broadley et al., 2005; Huang, Webb, Zourdos, \& Acevedo, 2013; Webb et al., 2011). Disorders associated with chronic stress may be clearly linked to the dysregulation of stress-response systems and their interconnected networks, including cardiovascular, metabolic and immune pathways (McEwen \& Wingfield, 2003). Prolonged secretion of primary mediators such as catecholamines and cortisol can cause damage to the brain and body (McEwen, 2006). Secondary outcomes ensue creating an imbalance between these interconnected networks as a result of the need to adjust and compensate to accommodate for the over and/or underproduction of stress hormones and other immune and cardiovascular system markers (Juster et al., 2010). The synergistic effects of the interaction between numerous primary mediators and secondary outcomes over time will create strain on the functioning of the 
organism, leading to a state of overload. When the system becomes chronically overloaded, tertiary outcomes will emerge in the form of cardiovascular (CVD), diabetes, and mortality (Juster et al., 2010, 2011).

Specifically, robust associations have been reported between chronic hyper-activation of stress systems and CVD (Rozanski, Blumenthal, \& Kaplan, 1999; Steptoe \& Kivimaki, 2012), mood disorder and anxiety (Kapczinski et al., 2008; McEwen, 2003), Cushing's disease (Starkman et al., 2001; Thomson et al., 2010), and cognitive impairment (Seplaki, Goldman, Weinstein, \& Lin, 2004). Similar to hyper-activation of the HPA system, hypo-activity is also associated with a heightened risk for development of hypotension, hypoglycemia, fibromyalgia, chronic fatigue, cognitive impairment, and autoimmune diseases such as Lupus, multiple sclerosis (Chrousos \& Kino, 2007; Tsigos \& Chrousos, 2002; Weiner, 1991), and Post-traumatic stress disorder (Steudte et al., 2013; Yehuda et al., 1990). Dysregulation of the HPA axis is strongly implicated in the pathology of depression (Carroll, 1982) as both hyper (Stetler \& Miller, 2011) and hypo-activity of the HPA axis (Lok et al., 2012) is documented.

Importantly, glucocorticoids can cross the blood-brain barrier, resulting in dendritic atrophy of hippocampal neurons and inhibition of neurogenesis in the dentate gyrus (Gould, McEwen, Tanapat, Galea, \& Fuchs, 1997), resulting in atrophy of brain tissue and subsequent emotional and cognitive impairments (Magarinos, \& McEwen, 1995; Sapolsky, 2000; Vyas et al., 2002). Exposure to increased circulating glucocorticoids over the lifespan could exacerbate these effects and interfere with healthy aging.

\subsection{Chronic Stress and Aging}

Exposure to chronic stress not only increases risk for negative health outcomes, such as CVD and mood disorders, but also is also associated with accelerated aging and early mortality 
(Miller, et al., 2007). Cumulative exposure to cortisol throughout the lifespan can lead to structural and cognitive changes in the brain, resulting in adverse effects on learning and memory with increasing age (Ferrari, et al., 2001; Lupien et al., 2005).

A seminal study by Issa and colleagues (1990) investigated the relationship between HPA dysfunction and cognitive impairment in young and aged rats. After subjecting all rats to the Morris water maze, aged rats were further divided into impaired aged rats and non-impaired aged rats, which performed as well as young rats on the spatial memory task. Not only was neuronal loss of hippocampal cells significantly greater in the impaired aged rats, $30 \%$ of the impaired aged rats further displayed increased basal HPA activity, relative to young and non-impaired aged rats. This increase in exposure to GCs was also correlated with reduced hippocampal volume, and subsequent memory performance. When the rat groups were exposed to an acute immobilization stress task, all groups reacted similarly; however, unlike the young and nonimpaired aged rats who displayed stress recovery following cessation of the stressor, the age impaired group maintained elevated GC levels, suggesting disruptions in the negative feedback loop (Issa et al., 1990). Changes in stress physiology as evidenced by basal hypersecretion of GCs, provides evidence of underlying individual differences in stress physiology and behavioral outcomes during the aging process.

In humans, some studies have reported elevated diurnal levels of cortisol in older adults, relative to younger adults (Chahal \& Drake, 2007; Epel, Burke, \& Wolkowitz, 2007; Nater, Hoppmann, \& Scott, 2013; Raskind, Peskind \& Wilkinson, 1994). Some research also suggests that aging is associated with heightened stress reactivity, evidenced by greater secretion of ACTH and cortisol in older adults compared with younger adults (e.g., Luz et al., 2003, Otte et al., 2005). However, other studies have shown that increases in basal cortisol are not necessarily 
an inevitable aspect of the aging process and do not represent a hallmark feature of the normal aging process (Kudielka et al., 2000; Waltman et al., 1991; Sherman et al., 1985). In a longitudinal study by Lupien and colleagues (1994) investigated cortisol levels over four years in a sample of healthy older adults. Similar to the aforementioned animal study by Issa et al., (1990), results showed individual differences in cortisol secretion with $30 \%$ of the sample displaying a consistent increase in cortisol each year and the remainder of the sample either displaying a consistent decrease each year or maintenance of cortisol levels over time (Lupien et al., 1994). Furthermore, the $30 \%$ of the sample who presented with currently high levels of cortisol accompanied by a steady year-to-year increase also showed significant impairment on a declarative memory and selective attention task (1994) and presented with a smaller hippocampal volume (1998), compared with the rest of the sample. Accordingly, differences in GC secretion over time can influence how cognitive decline presents in the aging process (Lupien et al., 1998). Although individual differences exist, it is suggested that normal agerelated changes in the central nervous system and brain areas that are integral to the stress response may heighten older adults' vulnerability to stress-related illnesses (Ferrari et al., 2001). More specifically, the amygdala, PFC, and hippocampus are documented to show the most rapid atrophy during the aging process, which are all brain areas sensitive to the effects of stress hormones (Lupien et al., 2009). Indeed, the PFC and hippocampus are brain structures that typically atrophy more rapidly with increasing age (Raz, et al., 2005). These structures are also specific targets for excess cortisol secretion, enhancing the effects of normal aging.

Taken together, research clearly shows that acute stress exposure can have positive effects on the organism as it ensures adaptation to a changing environment and survival. However, chronic stress exposure may have detrimental effects on the body and mind. Further, 
the impact of stress on the individual may become more taxing with increased age, as the effects of stress and aging are interactive, with stress exacerbating the effects of age (Hawkley \& Cacioppo, 2004; Sapolsky et al., 1986). In the context of an aging population, it is paramount to research non-invasive accessible interventions that facilitate stress management in both young and older adults. To this end, numerous studies have investigated the effects of music on wellbeing and stress recovery. However, before examining the current literature of music and well-being, it is important to briefly discuss how stress physiology is examined in the laboratory. 


\section{CHAPTER THREE}

\section{Stress Induction and Measurement}

\subsection{Measurement of Stress Physiology}

Within the current stress literature, researchers have investigated responses to real-life stressors such as bereavement, job stress, natural disasters, or during academic examinations (for review, see Biondi \& Picardi, 1999). Although the measurement of naturally occurring stressors can be considered more ecologically valid, extraneous and confounding variables within the environment can make interpretation of findings difficult.

Over the past few decades, a number of laboratory stress induction paradigms have been developed. The two most widely used and validated stress induction paradigms include the Cold Pressor Test, a physical stressor (CPT; first described by Hines \& Brown, 1932), and the Trier Social Stress Test (TSST; Kirschbaum, Pirke, \& Hellhammer, 1993), a psychosocial stressor. The standard CPT procedure involves submerging a participant's non-dominant hand up to the wrist in ice water (typically between $0-4^{\circ} \mathrm{C}$ ) for a duration ranging between 1 to 5 minutes (Blandini et al., 1995; Hines \& Brown, 1932; McRae et al., 2006). This task is designed to be physically uncomfortable, even painful to some.

While the CPT is found to reliably increase sympathetic activity, as documented by increased skin conductance levels (Buchanan, Tranel, \& Adolphs, 2006) and elevated blood pressure (al'Absi et al., 2002), the effects of CPT on HPA activation is less clear. Some studies have reported the CPT to activate the HPA system (Pascualy et al., 2000; Schwabe, Haddad, \& Schachinger, 2008), while others have failed to report elevated levels of cortisol in response to the task (Duncko et al., 2007; McRae et al., 2006). It has been postulated that the relative weak induction of HPA responsivity by the CPT is due to the lack of social challenge or evaluative threat, which as mentioned above is one of the ingredients to stress (i.e., $\mathrm{T}$ of the NUTS model). 
This hypothesis was supported by Schwabe and colleagues (2008), who tested the differential effect of a modified CPT, which incorporated a social-evaluative threat by telling participants that they were being videotaped and were closely watched by the experimenter during the task. Results showed that the modified CPT resulted in a significantly higher peak cortisol concentration 20-30 minutes following stress induction compared with the standard CPT protocol (Schwabe et al., 2008). Similarly, CPT and the TSST have been compared in their relative activation of the HPA axis. In a study by McRae et al., (2006), plasma ACTH and cortisol levels were collected as markers of HPA activity in a group of participants who completed the CPT, and also underwent the TSST approximately 2 months later. Results demonstrated that peak levels of both ACTH and cortisol were significantly higher following completion of the TSST relative to the CPT (McRae et al., 2006). These results demonstrate the importance of incorporating a social-evaluative threat component in protocols assessing HPA activity.

The most widely used standardized psychosocial stressor is the TSST, which incorporates a high level of uncontrollability and social-evaluative threat (Kirschbaum et al., 1993; Dickerson \& Kemeny, 2004; Lupien et al., 2013; Mason, 1968). Briefly, the TSST protocol involves an anticipation period, where participants are told that they are to prepare a 5-minute speech on a predetermined topic (usually a mock job interview), followed by presentation of the 5-minute speech in front of a video camera and a panel of research confederates who are presented as "experts" or "evaluators" and who are instructed to be non-responsive and indifferent to the participant's performance. Following the 5-minute speech component, participants are informed of a "surprise" 5-minute serial subtraction arithmetic task, which involves beginning with a large number and counting backwards by a prime number (e.g., 7 or 13). If the participant makes a 
miscalculation, the panel stops the participant and instructs them to start from the beginning. Together, components of the TSST induce a high level of social-evaluative threat and uncontrollability (Allen, et al., 2017).

Numerous studies have demonstrated the effects of the TSST on indices of stress reactivity in both the SAM and HPA axes (Allen et al., 2014). For instance, studies have shown that exposure to the TSST increases sympathetic activity through observed increases in heart rate (Jezova et al., 2004; Rimmele et al., 2007, 2009), skin conductance (Jezova et al., 2004;

Rohrmann et al., 1999), and secretion of catecholamines (Gold et al., 2004; Schommer et al., 2003). Further, strong support within the literature demonstrates that exposure to the TSST stimulates the HPA axis as measured by increases in ACTH (Kumsta et al., 2007; Munro et al., 2005) and cortisol secretion (Dickerson \& Kemeny, 2004; Het et al., 2009; Gaab et al., 2005; Kirschbaum et al., 1993, 1999). Importantly, the TSST demonstrates similar effects on stress physiology across the lifespan including children, adolescents, young adults, middle-aged adults (Kudielka et al., 2004; Gunnar, Talge \& Herrera, 2009), and older adults (Kudielka et al., 1998, 2000), demonstrating its effectiveness and suitability as a robust stress induction protocol that target both SAM and HPA activation.

3.1.1. Indices of SAM activation. Evolutionarily speaking, when a stressor is perceived to be threatening, respiration and heart rate are increased in order to send oxygenated blood to parts of the body needed to react to the threat. Sympathetic activation also increases perspiration from the eccrine glands to the pores of the skin for thermoregulation, to maintain core body temperature in the face of threat and once the threat subsides (Dawson, Schell, \& Filion, 2007). Both HRV and skin conductance activity are considered to be two reliable, non-invasive markers of SAM activation. 
HRV is the variation in heart rate, measured through the interval between consecutive heart beats, referred to as the inter-beat interval (RR; Bernston, 1997; Task Force of the European Society of Cardiology, 1996; Thayer et al., 2012). The heart is stimulated by both the sympathetic (increases in heart rate) and parasympathetic system (decreases in heart rate) with relative increases resulting in shorter or longer inter-beat intervals respectively (Thayer et al., 2012). A recent meta-analysis implicated HRV to be a reliable index of stress through reported associations with brain structures involved with the perception of threat, such as the amygdala and PFC (Thayer et al., 2012). A number of studies document lower HRV when exposed to acute mental and/or psychosocial stress relative to control conditions (Hall et al., 2004; Hjortskov et al., 2004); and when perceiving a task as being stressful (e.g., Dishman et al., 2000). Despite documented individual variability (Fohr et al., 2015; Task Force, 1996), HRV is suggested to be a consistent indicator used to interpret physiological or objective effects of exposure to stress (Van Amelsvoort et al., 2000).

Skin conductance activity is determined through measurement of the changes in sweat gland activity on the surface of the skin in response to an arousing stimulus or event (Cacioppo, Tassinary, \& Berston, 2007; Harrison et al., 2006). Studies have reported significant increases in skin conductance activity following exposure to both physical (Harrison et al., 2006) and psychological stressors (Allen, Blascovich, Tomaka, \& Kelsey, 1991; Liu, Verhulst, Massar, \& Chee, 2015).

3.1.2. Indices of HPA activation. As mentioned previously, the final product stemming from HPA activation is the secretion of cortisol, which is the gold standard for measuring HPA reactivity (Hellhammer, Wust, \& Kudielka, 2008). Although urinary cortisol provides an index of HPA activity (Baum \& Grunberg, 1995; King \& Hegadoren, 2002), blood and salivary 
cortisol are common markers of HPA reactivity (Gunnar \& Donzella, 2002).

Cortisol measured in blood was the first technique used to measure cortisol secretion in response to a stressor; however, the method is relatively intrusive as it involves venipuncture, which is shown to cause a stress-response itself and artificially increase levels of cortisol throughout a stress induction protocol (Kudielka, et al., 2012; Weckesser et al., 2014). With the development of new measurement techniques and assays, salivary cortisol measurement has become a more common practice as it is less invasive and provides a more accurate measurement of the "active" hormone (Nater \& Rohleder, 2009).

Approximately $10 \%$ of cortisol is unbound and biologically active, whereas $90 \%$ of cortisol remains bound to proteins (Gunnar \& White, 2001). The biologically active hormone is the portion that modulates cellular function and crosses the blood brain barrier to induce neuronal changes (Juster et al., 2010). Extraction of cortisol from blood includes both bound and unbound cortisol; whereas extraction from saliva only measures unbound, free cortisol. Although there is a strong correlation between cortisol levels measured in saliva and blood (Hellhammer et al., 2008; Kudielka et al., 2012), it is considered more important to estimate the amount of free cortisol secreted as it is this unbound hormone that has a direct effect on allostasis of the organism (McEwen, 2007).

Given the diurnal pattern of HPA activity and cortisol, with a peak in the morning hours and a steady decline throughout the day, resulting in nadir phase at night (Baum \& Grunberg, 1995), stress induction protocols are commonly employed in the afternoon to avoid potential ceiling effects of cortisol reactivity (Pruessner et al., 1997; Kudielka \& Kirschbaum, 2003). Cortisol concentrations in saliva peak shortly after awakening, with expected levels at approximately $0.5 \mu \mathrm{g} / \mathrm{dL}$. It is more ideal to sample cortisol in response to acute stress in the early 
- late afternoon because the concentration of cortisol is moderate at approximately $0.3 \mu \mathrm{g} / \mathrm{dL}$. Sampling during this time allows for the capture of a more representative basal level of cortisol within the participant by avoiding the heightened CAR, and the lowest concentration later at night prior to sleep that may artificially increase or decrease the cortisol response to the TSST.

Reactive cortisol secretion in response to a stress induction protocol has also found to display a relatively typical profile, with a rise in cortisol secretion 10-20 minutes following acute stress induction, following by a recovery period (Gaab et al., 2005; Het et al., 2009; Petrowski et al., 2010). However, other studies have reported a wider range in peak concentrations occurring between 20-40 minutes (Dickerson \& Kemeny, 2004). Further, following anticipation of a psychosocial stressor, salivary cortisol levels have been observed to begin increasing between 14 and 20 minutes (Engert et al., 2013). Given the variation in time in which peak salivary cortisol levels can be observed, it is absolutely crucial to include multiple sampling time points within a stress-induction protocol in order to fully capture the entire trajectory of the cortisol response.

Together, the aforementioned stress indices enable researchers to gain insight into the functionality of the SAM and HPA systems when faced with an acute stressor. To gain a comprehensive picture of how music exposure may inoculate the stress response, this dissertation will employ the TSST and include measures of HRV, skin conductance and salivary cortisol secretion. 


\section{CHAPTER FOUR}

\section{Music and Well-being}

\subsection{Beneficial Effects of Music}

Music is a powerful and complex stimulus. Over the past few decades, music research has gained increasing attention in the areas of psychology and neuroscience due to its wide range of effects on cognition, emotion, and well-being.

Listening to music can regulate emotion (Chanda \& Levitin, 2013; Juslin \& Laukka, 2004; Sloboda \& O'Neill, 2001) and can produce feelings of intense emotion (Zentner, Grandjean, \& Scherer, 2008). Qualitative studies in populations of healthy young adults provide evidence that music can regulate mood and facilitate pleasure and relaxation (North et al., 2004; Sararikallio \& Erkkila, 2007; Sloboda, O’Neill, \& Ivaldi, 2001). Music has also been found to enhance physical performance. In a study by Terry, Karageorghis, Saha and D'Auria (2012) triathletes were monitored while running in synchrony to either motivational self-selected music, neutral music, or no music (Terry et al., 2012). Results revealed that when running to selfselected music or neutral music, athletes maintained their stamina for a longer duration compared to the no-music condition. Further, self-reports of positive affect remained higher while running to self-selected music relative to the other two conditions (Terry et al., 2012).

Qualitative and quantitative studies in populations of healthy older adults provide converging evidence that music may have beneficial effects by enhancing social cohesion and facilitating maintenance of well-being (Creech, Hallam, McQueen, \& Varvarigou, 2013; Laukka, 2007). For example, participating in active music making through senior orchestras has been shown to increase feelings of belonging, enjoyment, and happiness that were directly attributed to their engagement in music (Gembris, 2008). Further, in a study by Coffman and Adamek (1999), it was found that older adults who participated in a wind music ensemble reported 
increased wellbeing and quality of life. Passive music listening is also beneficial for older adults. Hays and Minichiello (2005) showed that active participation or passive listening of music both incurred positive outcomes, including feelings of enhanced quality of life, emotional well-being, positive self-esteem, and decreased perceptions of loneliness and isolation.

Music has also shown to be beneficial in older adult clinical samples, of patients with Alzheimer's disease (AD). In a randomized control trial, Clement and colleagues (2012) examined the short and long term effects of a 4-week music intervention (music listening or music making) or cooking intervention (tasting cake or preparing a recipe), in patients with severe $\mathrm{AD}$. While the cooking intervention resulted in some short-term positive effect on mood, the music intervention showed significant short and long-term enhancement of positive mood state among patients (Clement et al., 2012). In addition to increasing positive affect, self-selected music has also been documented to reduce the frequency of agitated behavior in patients with AD compared to experimenter-selected classical music (Gerdner, 2000).

\subsection{Neural Correlates of Music Emotion}

Imaging research shows that the production and perception of music activates and engages cortical and sub-cortical brain regions (Menon \& Levitin, 2006), including neuronal regions involved in reward and pleasure (Blood \& Zatorre, 2001; Menon \& Levitin, 2005). More specifically, when listening to music considered to be emotionally moving, enhanced activation is found in areas of the PFC (Limb, 2006; Menon \& Levitin, 2005), an important brain area involved in emotion regulation and critical for integration of emotion and sensory information through reciprocal connections to the limbic system. Furthermore, using functional magnetic resonance imaging (firm), Koelsch et al., (2006) revealed differential activation patterns as a function of music valence. Specifically, significant activation of the insula (important for 
regulation of autonomic functions (Critchley, 2005)), and ventral striatum (important for reward processing (Kelley, 2004)) occur when listening to music considered to be pleasant. However, when listening to unpleasant music, greater activation is observed in the amygdala, hippocampus and temporal poles (Koelsch, et al., 2006). Given that activation and communication between the amygdala and hippocampus occur in response to stress, it may be suggested that music listening can influence the physiological circuitry associated with the stress response.

\subsection{Psychophysiology of Music and Stress Reactivity}

Music has received increasing attention as a non-invasive, inexpensive way to reduce stress. Listening to, or actively making music has been shown to activate the autonomic nervous system. For instance, music characterized as highly arousing (e.g., faster tempo, and very loud) shows increased sympathetic activity as measured by increased skin conductance, respiration, heart rate, blood pressure, and vigilance (Khalfa et al., 2002; Nater, Abbruzzese, Krebs, \& Ehlert, 2006). Conversely, music characterized as relaxing (slow tempo, low pitch, and is typically classical without lyrics) can activate the parasympathetic nervous system by attenuating skin conductance (Sandstrom \& Russo, 2010; Sokhadze, 2007), heart rate, blood pressure, and respiration (Knight, \& Rickard, 2001). Given the modulatory role of music on autonomic function, research has investigated whether music can modulate stress reactivity to a stressor.

Results from multiple experimental studies indicate beneficial effects of music on stresssensitive systems following stress induction (Khalfa et al., 2003; Suda et al., 2008). In examining the effect of music listening on SAM activity, Sandstrom and Russo (2010) investigated whether music valence and arousal can influence skin conductance levels while recovering from an acute stressor. Eighty-three undergraduate students were randomized into 5 experimental conditions: peaceful music, happy music, sad music, agitating music, and white noise control. Before music 
exposure, all participants watched a short news clip and were later told that they had to present a short speech on the clip. Participants were given one minute to prepare the speech, which served as the stress inducer. Following stress induction, participants were told that they were not actually required to give a speech, and were asked to listen to a music clip, based on the condition to which they were randomized to. Skin conductance level and heart rate were continuously recorded throughout the experiment, and subjective responses regarding level of discomfort and liking of music were obtained.

Results demonstrated that the peaceful music condition facilitated faster skin conductance recovery following stress induction compared with the sad music, happy music, agitating music, and white noise conditions (Sandstrom \& Russo, 2010). Heart rate data showed a similar pattern such that music that was peaceful facilitated faster recovery compared to the white noise control condition (Sandstrom \& Russo, 2010). The recovery time course for the white noise condition was comparable to the happy, sad, and agitated music conditions, as evidenced by similar HR patterns following anticipation of the stressor. These results provide important insight into how music type can influence physiological recovery from acute stress. More specifically, as individuals in the relaxing music group recovered more quickly relative to the other groups, this demonstrates that not all music genres or types are equally beneficial in reducing stress. This provides support that the specific features or parameters of relaxing music such as a slow tempo, major mode, soft timbre, and a narrow pitch range (Justin \& Laukka, 2004), can be used to model and develop effective stress reduction interventions.

In examining the effect of music listening on HPA function, Khalfa and et al., (2003) assessed whether relaxing classical music could facilitate recovery from an acute psychosocial stressor. A small sample of male undergraduate students $(N=17)$ was subjected to a 
standardized laboratory psychosocial stressor, after which they were randomized to a music listening condition or a silence condition. Results demonstrated that both groups experienced a significant increase in cortisol level following acute stress induction; however, the music condition displayed a quicker recover from the stress induction protocol, evidenced by lower cortisol levels within the initial recovery phase (first 15-20-minute period following stress induction). Although this particular study had a small sample size, the findings are in line with research that suggests music listening decreases stress, as evidenced by reductions in cortisol levels prior to (Milukkolasa, Obrniski, Sputnicki, \& Golec, 1994; Ventura et al., 2012), or following surgery (Nilsson, Unosson, \& Rawal, 2005).

Despite these positive findings, some studies have reported increases in cortisol in response to music listening (Escher et al., 1993; Uedo et al., 2004), while others report no significant effect of music on cortisol secretion following acute stress (Chlan et al., 2007; Conrad et al., 2007; Knight \& Rickard 2001; Yamamoto, Naga, \& Shimizu, 2007). An important limitation of past literature looking at music's ability to reduce stress is that researchers largely ignore, or fail to incorporate, individual difference factors that may determine stress reactivity and recovery in response to music listening.

\subsection{Individual Differences in Music Selection and Music Absorption}

The majority of research that investigates the benefits of music on recovery from a stressor use "relaxing classical music". However, perception of what is considered relaxing may differ across individuals, which poses a methodological limitation as genre or type of music may moderate the effectiveness of music listening on the stress response.

Illustrating how music genre and personal preference differentially effect recovery following stress exposure, Labbé and colleagues (2007) collected physiological (heart rate, 
respiration, and skin conductance) and self-report measures of anxiety and relaxation from a sample of 56 undergraduate students who listened to different types of music (experimenterselected classical, experimenter-selected heavy metal, or self-selected relaxing music) or sat in silence following administration of an acute cognitive stressor. Results of the study showed that participants who listened to self-selected relaxing music experienced greater decreases in state anxiety, increases in reports of relaxation, and reductions in HR relative to the heavy metal group and the silence condition (Labbé et al., 2007). However, when examining the skin conductance response, all groups experienced a significant reduction in skin conductance.

In a study that examined self-report anxiety following acute psychological stress as a function of arousal and personal preference for music, Jiang, Zhou, Rickson, and Jiang (2013) asked participants to choose one preferred piece and one non-preferred piece of music from a prepared list of classical Chinese songs. Participants were randomized to one of four music groups (preferred stimulating music, preferred sedative music, non-preferred stimulating music, or non-preferred sedative music). Following stress induction employing a brief mental arithmetic task, participants listened to music in accordance with their randomized group. The authors hypothesized that listening to preferred music would result in lower reports of tension and anxiety compared to those who listened to non-preferred music. Results showed a music type by music preference interaction on subjective perceptions of tension following the acute stressor. More specifically, when participants listened to un-preferred music, those listening to sedative music reported lower state-anxiety and tension compared to participants who listened to stimulating music. However, when music was preferred, there was no difference between sedative and stimulating music exposure, suggesting that preference for music type is more important than music type alone. (Jiang et al., 2013). However, because this study only measured 
subjective appraisals of tension and anxiety, it is difficult to conclude if this effect would also be observed in physiological indices of stress.

Another individual difference factor that may potentially moderate the effect of music on stress is music absorption. Generally, the trait of absorption can be defined as the ability to focus attention on, or the ability to fully engage in one particular stimulus, while ignoring other stimuli within that environment (Wild, Kuiken, \& Schopflocher, 1995). Degree of absorption is typically measured using the Tellegen Absorption Scale (TAS; Tellegen \& Atkinson, 1974), a scale originally developed to identify the degree to which an individual may be susceptible to hypnosis. Music has often been associated with such experiences in altered states of consciousness such as meditation, and trance states (Hills \& Argyle, 1998). Positive associations have been documented between TAS scores and the ability of music to influence emotions (Wild et al., 1995). Music absorption, specifically, can be conceptualized as the degree to which an individual has the capacity to become absorbed, or immersed while listening to, or playing music. However, there is a lack of research investigating any modifying effects of music absorption in the context of stress.

Using data collected in a previous study (Sandstrom \& Russo, 2010), Sandstrom and Russo examined the role of absorption as an individual difference factor in the relationship between music listening and recovery from an anticipatory stressor (Sandstrom \& Russo, 2013). Results showed that absorption, as measured by the TAS, was a significant predictor of physiological recovery when listening to peaceful music, but not when listening to white noise. More specifically, high absorbers recovered more quickly than individuals with low absorption scores in the peaceful music condition, whereas absorption level did not influence the duration of time to recover from stress in the white noise condition (Sandstrom \& Russo, 2013). Stemming 
from this study, a 34-item Absorption in Music Scale (AIMS) was developed to measure degree of absorption when listening to music, thus identifying individuals who may be particularly responsive to music (Sandstrom \& Russo, 2013). In support of this, Hogue, Crimmins, and Kahn (2016) showed that music absorption in young adults was positively associated with liking music that induces both happiness and sadness. In a questionnaire-based study by Loxton, Mitchell, Dingle, and Sharman (2016) that examined the role of music engagement in Reinforcement Sensitivity Theory, it was found that the positive association between reward sensitivity and music involvement was mediated by music absorption. Accordingly, music absorption may be an important individual difference factor that should be considered when examining the effectiveness of music on inoculating the stress response.

Taken together, it is postulated that individual differences in music selection and music absorption may moderate the potential effect of music listening on the stress response. However, more research is needed to investigate these moderating effects. 


\section{CHAPTER FIVE}

Study Objectives

\subsection{Gaps in the Current Literature and Study Objectives}

To date, the majority of research has focused on the benefit of music listening to facilitate recovery following stress exposure. However, there is a paucity of research that evaluates whether music presented prior to an acute stressor may inoculate stress reactivity to future stress exposure. A recent study by Thoma et al. (2013) sought to address this question in a sample of 60 healthy adult females. Participants were randomly assigned to one of three experimental conditions: relaxing classical music, sound of rippling water, or a no acoustic simulation control group. Following randomization and music listening for 10 minutes, participants underwent the TSST to induce acute psychosocial stress. Salivary cortisol, heart rate, and self-reports of anxiety and distress were repeatedly assessed at different time-points throughout the experiment. It was hypothesized that the relaxing music group would experience the lowest level of responsivity across all physiological outcomes. Surprisingly, results showed that the highest concentration of cortisol was observed in the relaxing music group, and the lowest in the rippling water group. Although the author's hypothesis was not supported, this study presents with two important limitations: choice of music and sample characteristics. First, the relaxing music condition consisted of a piece of classical music chosen by the researchers, a Latin choral selection, which has a relatively ominous sound. This highlights the importance of individual differences in music preference. Since the highest concentrations of cortisol was observed in the relaxing music condition relative to the rippling water control condition, it is possible that this particular music selection was simply not ideal for inducing feelings of relaxation, and that the rippling water was more relaxing by comparison. Although standardizing the music within the relaxing music condition by using a single piece of music may provide the researcher with more control, it does 
not allow for any assessment of personal preference of music, and how that may moderate the participant's physiological and subjective responses to acute stress. Second, it is difficult to generalize the results, as the sample comprised of women with a mean age of 25 years. Robust gender differences in cortisol secretion and reactivity have been noted; specifically, young adult men exhibit higher levels of cortisol relative to women during anticipation of a stressor (Kirschbaum, Wust, \& Hellhammer, 1992; Seeman, Singer, Wilkinson, \& McEwen, 2001). Thus, inclusion of men in the sample needs to be incorporated in future studies.

While the majority of research investigating stress-reducing effects of music is conducted with populations of younger adults, there is a paucity of research that investigates music's effectiveness in reducing stress in healthy older adults, a growing segment of the population. As mentioned above, research documents that active participation in music can enhance the quality of life and well-being in older adults (Creech et al., 2013). However, it is not clear whether music can inoculate physiological stress in this population. Due to the potential for cumulative exposure to chronic stress across the lifespan, the older adult population is at a heightened risk for development of adverse health outcomes and pathology. As a result, research investigating and assessing music as a potential stress inoculator is paramount.

\subsection{Research Aims and Hypotheses}

To address previous study limitations and expand on the literature, this study examined the inoculating effect of music listening prior to psychosocial stress induction on stress reactivity in young and older adults and further examined the moderating role of music selection and music absorption. This study provides a relatively comprehensive approach to examine whether music exposure prior to stress induction can inoculate the stress response across stress-sensitive systems, the SAM and HPA axis. To the author's knowledge, this is the first study to investigate 
the inoculation effect of music in both young and older adults. As discussed above, research has been limited to young populations and sometimes to a single sex (e.g. females only). Further, this is the first study to evaluate music selection and music absorption as potential modulators of music's effect in attenuating the stress response in both young and older adults. To this end, the objectives of the current study were three-fold:

1) To determine whether music exposure before an acute psychosocial stressor decreases stress reactivity. Hypothesis: It was hypothesized that individuals who are exposed to music prior to an acute stressor will display lower stress reactivity compared to individuals exposed to white noise, evidenced by lower cortisol secretion, lower skin conductance and increased HRV.

2) To determine whether selection of music determines the "inoculation effectiveness" of music on stress reactivity. Hypothesis: It was hypothesized that self-selected music will diminish the stress response more than experimenter-selected music.

3) To determine whether music absorption moderates the inoculation effect of music on stress reactivity. Hypothesis: It was hypothesized that the inoculation effect of music listening and self-selected music would be greater in high music absorbers compared to low absorbers.

Exploratory analyses were conducted to determine whether the aforementioned associations differ in young and older adults. 


\section{CHAPTER SIX}

\section{Method}

\subsection{Participants}

According to power analysis using GPower 3.0 software (Faul, Erdfelder, Lang, \& Buchner, 2007), a total of 54 participants was required to find a significant effect of condition on stress reactivity. This sample size was based on an effect size of $d=.15$, assuming $80 \%$ power at an alpha level of 5\%. This effect size was based on a study by Thoma et al, (2013) who examined the effect of music exposure on cortisol reactivity. To account for potential nonresponders or equipment malfunction, the total sample size was corrected for $20 \%$ potential lost data, resulting in a total sample size of 64, with 21 participants per group.

Sixty-one young adults $(\text { Mage }=23.83, S D=4.23)^{1}$, and 46 older adults $($ Mage $=66.72$, $S D=4.73$ ) were recruited through online advertisements (i.e., Kijiji and Craig's list), advertisement flyers distributed in the community (e.g., message board at a community center, libraries, or coffee shops), and from Ryerson University participant pools (i.e., Ryerson Psychology Research Pool and the Ryerson Senior Participant Pool $)^{2}$. Individuals who responded to the study advertisement were provided with details of the study purpose and procedure during a telephone call. Refer to Appendix A for the telephone script.

The young adult sample was characterized as $70.5 \%$ female, $33 \%$ Caucasian, with $34 \%$ reporting some university education, and $61.7 \%$ reporting medium socioeconomic status (SES). The older adults sample was characterized as $55.1 \%$ female, $75.5 \%$ Caucasian, with $34.4 \%$

\footnotetext{
${ }^{1}$ Of the 61 younger adult participants included in the final sample, 5 did not provide their age. The mean age is based on 56 individuals within this sample.

2 The first fourteen younger adult participants were recruited from the Ryerson Psychology Research Pool (SONA) until funding was available to compensate eligible age-matched community members and students (remaining 46 participants).
} 
reporting to have attended university, and $63.3 \%$ reporting medium SES. See Table 1 for all sample characteristics.

Exclusion criteria for the study included use of certain medications that interfere with HPA activity such as cortisone, hormone replacement therapy, or antidepressant/antipsychotic medication (e.g., Granger et al., 2009; Kudielka et al., 2012). Participants were also excluded if they indicated any of the following conditions: uncontrolled high blood pressure, uncontrolled cardiovascular disease, current pregnancy, diabetes, seizure disorder, diagnosed sleep disorder, thyroid disorder or other endocrine system conditions, and a clinical diagnosis of anxiety and/or depression. Female participants who were taking low-dosage oral contraceptives, which are known to influence HPA activity, were included in this study and controlled for in the statistical analysis $^{3}$. Older female participants included in the study were post-menopausal and not taking hormone replacement therapy (see Appendix B for eligibility questionnaire).

\subsection{Musical and Acoustic Control Stimuli}

6.2.1. Researcher-selected music. A pilot study was conducted to select an appropriate musical piece for the RSM condition; one which would be considered relaxing and peaceful. A convenience sample of 17 Ryerson graduate students not affiliated with the current study volunteered to complete a short online survey using Qualtrics@ where they were asked to rate seven different classical music pieces ( 10 minutes each) based on how they felt both physiologically (arousal) and emotionally (valence) while listening. The particular pieces included were chosen either based on their use in existing literature, or at the researcher's discretion. Response items for valence were "very or somewhat relaxed", "very or somewhat

\footnotetext{
${ }^{3}$ Female participants were asked to indicate the phase in their current menstrual cycle in order for this to be controlled for during analyses. Women in the luteal phase of the menstrual cycle have been shown to exhibit greater responses to the TSST relative to women in the follicular phase (e.g., Childs, Dlugos, \& De Wit, 2010).
} 
agitated", and "neutral". Response items for valence were "very or somewhat peaceful", "very or somewhat sad", and "neutral". See Table 1 for pilot ratings of relaxation and peacefulness.

Given that the main goal was to maximize relaxation, while still peaceful, Shostakovich's Piano Concerto \#2 in F major, andante (moderately slow tempo) was chosen as $94.1 \%$ of participants considered it to be relaxing and no one rated the piece of music as agitating. The music piece was downloaded using Apple iTunes $\odot$ and normalized using the root mean square to ensure for equal sound intensity. Given that the volume of auditory stimuli has been positively associated with arousal levels (Huron, 1992), and levels exceeding 70 decibels (dB) have the potential to increase cortisol secretion (Ising \& Braun, 2000), the volume was pre-set as to not exceed $70 \mathrm{~dB}^{4}$.

6.2.2. Self-selected music. Prior to the lab visit, participants randomized to the selfselected music condition were contacted via email and asked to provide a list of three songs that they liked, and that they would consider listening to if they wanted to relax. Participants were free to choose from any genre in order to emphasize music preference. Using the digital music service Spotify Premium, a playlist lasting 10-minutes was created containing their chosen songs. As each playlist was made, the song quality was checked to ensure the recording wasn't noisy, and the volume was pre-set between 60-70dB.

6.2.3. White noise control. White noise was chosen as an acoustical control stimulus. White noise has been used in past studies (Sandstrom \& Russo, 2010; Sokhadze, 2007), because it is considered to be relatively emotionally neutral while maintaining auditory stimulation (Nyklicek, Thayer, \& Van Doornen, 1997). A free white noise audio file with a 10-minute

\footnotetext{
${ }^{4}$ A quick volume test was performed with each participant prior to induction to ensure that the pre-set volume was adequate for each individual. Participants were instructed not to adjust the volume themselves, but to ask the researcher if needed. Any participants that adjusted the volume were noted.
} 
duration was downloaded from the website http://mc2method.org/white-noise/ and RMS

normalized. Volume was also pre-set by the research as to not exceed $70 \mathrm{~dB}$.

Table 1. Pilot Study (n=17) on Arousal and Valence of Researcher-Selected Music

\begin{tabular}{lll}
\hline $\begin{array}{l}\text { Song title } \\
\text { (Musician) }\end{array}$ & Arousal Ratings & Valence Ratings
\end{tabular}

\begin{tabular}{|c|c|c|c|c|c|c|}
\hline & $\begin{array}{c}\text { Relaxing } \\
(\%)\end{array}$ & $\begin{array}{c}\text { Agitating } \\
(\%)\end{array}$ & $\begin{array}{c}\text { Neutral } \\
(\%)\end{array}$ & $\begin{array}{c}\text { Peaceful } \\
(\%)\end{array}$ & $\begin{array}{l}\text { Sad } \\
(\%)\end{array}$ & $\begin{array}{c}\text { Neutral } \\
(\%)\end{array}$ \\
\hline $\begin{array}{l}\text { Nuvole } \\
\text { Bianche } \\
\text { (Ludovico } \\
\text { Einaudi) }\end{array}$ & 82.4 & 16.67 & 0 & 64.7 & 22.22 & 11.11 \\
\hline $\begin{array}{l}\text { Divenire } \\
\text { (Ludovico } \\
\text { Einaudi) }\end{array}$ & 47.1 & 22.22 & 5.56 & 64.7 & 16.67 & 22.22 \\
\hline $\begin{array}{l}\text { Piano } \\
\text { Concerto No. } \\
5 \text { in E-flat } \\
\text { Major, Op. } 73 \text {, } \\
\text { Adagio } \\
\text { (Beethoven) }\end{array}$ & 76.5 & 11.11 & 11.11 & 58.8 & 27.78 & 11.11 \\
\hline $\begin{array}{l}\text { Piano } \\
\text { Concerto No. } \\
\text { 2-II Andante } \\
\text { (Shostakovich) }\end{array}$ & 94.1 & 0 & 5.56 & 58.8 & 16.67 & 11.11 \\
\hline $\begin{array}{l}\text { Claire De } \\
\text { Lune } \\
\text { (DeBussy) }\end{array}$ & 82.4 & 5.56 & 0 & 76.5 & 11.11 & 11.11 \\
\hline $\begin{array}{l}\text { Le Onde } \\
\text { (Ludovico } \\
\text { Einaudi) }\end{array}$ & 64.7 & 22.22 & 11.11 & 64.7 & 27.78 & 27.78 \\
\hline $\begin{array}{l}\text { Piano } \\
\text { Concerto in A } \\
\text { Minor, Op. 16- } \\
\text { II, Adagio } \\
\text { (Edvard } \\
\text { Grieg) }\end{array}$ & 70.6 & 5.56 & 22.22 & 52.9 & 16.67 & 27.78 \\
\hline
\end{tabular}




\subsection{Measures}

Validated objective and subjective measures were used in the present study. Responsivity to acute psychosocial stress was measured using electrophysiological, biochemical and subjective measures. Additional psychological questionnaires were administered to assess music absorption, mood and personality traits.

6.3.1. Electrophysiological measures. The BIOPAC BioNomadix wireless system with MP150 data acquisition was used for measurement of physiological indices. Physiological data was sampled at an acquisition rate of 2000 samples per second, with a gain of 2000.

Physiological measures including skin conductance level (SCL), skin conductance response (SCR), heart rate (HR), and pulse rate were collected continuously throughout the experiment. Electrodermal activity (EDA) is a term that encompasses both the SCL, or background tonic, and SCR, the rapid phasic components that emerge as a result of SNS activity in response to a psychological challenge (Dawson et al., 2007). The SCL measure reflects the more general changes in autonomic arousal over time, whereas the SCR measure represents the faster changing elements of the signal and is quantified by counting the number or peak responses. SCL can vary between and within individuals, but the typical level of activity can range from $2 \mu \mathrm{S}$ (microsiemens) - 20 $\mu \mathrm{S}$ (Dawson, et al., 2007). The frequency of SCRs are typically estimated to occur 1-3 times per minute, however, this can increase to approximately 20-25 per/min when exposed to situations inducing high levels of arousal (Boucsein, 2012; Braithwaite, et al., 2013). EDA is a widely used objective marker of sympathetic arousal and has been used in previous studies that employ the TSST as a means of psychosocial stress induction (Braithwaite et al., 2013; Allen et al., 2014). Although some debate exists in the literature, (see Boucsein, 
2012) inclusion of both SCL and SCR should not be considered direct replacements for each other, as both provide valid, and distinctly different measurements of physiological arousal (Braithwaite et al., 2013).

In order to measure EDA, two electrodes were placed on the middle and ring finger of the participant's non-dominant hand in order to detect any changes in physiological arousal during the testing session and during time-intervals of interest. The ground electrode was always attached to the middle finger across participants to maintain consistency. When necessary, additional gel was applied to each electrode in order to optimize signal conductance. On the index finger of the same hand, the pulse plethysmograph (PPG) monitor was attached to record their pulse rate throughout the experiment.

Electrocardiography (ECG) was used to measure the electrical activity of the participant's heart continuously throughout the experiment. Three electrodes were placed on the participant in order to create a closed-circuit to capture heart rate (HR) activity, measured by total beats per minute; one directly under the right clavicle, one directly below the left rib cage, and one directly below the right rib cage (ground electrode). Heart rate variability (HRV) is an objective measurement of the variations between heartbeats, and can be extracted from the ECG recording. HRV has been shown to correlate with psychological stress (Berntson et al., 1997; Thayer et al., 2012), and thus, is considered to be a reliable marker of both SNS and PNS activity in this context (Acharya et al., 2006). Although HRV, may be considered to be a more sensitive index of cardiac ANS function (Kleiger et al., 2005), the mean HR also provides valuable information regarding cardiac function in response to acute stress, and has been used in other studies employing the TSST (Kudielka et al., 2004; von Dawans, Kirschbaum, \& Heinrichs, 2011). 
All raw physiological data collected during the experiment was visually inspected, filtered for artefacts, processed, extracted, and analyzed using AcqKnowlege software. Regions of interest (baseline, music induction, anticipation, TSST, and 4 10-minute recovery periods) were visually inspected for artefacts, extracted, and processed, using the AcqKnowlege software. Any sections of the data that were corrected as a result of obvious artefact (i.e., a drop in the signal due to equipment failure) during visual inspection was done using a transform function within AcqKnowlege called "connect endpoints". This particular function was used in order to avoid creating time shifts in the data when correcting for artefacts, and it substitutes the selected data with a linear interpolation based on values of the left and right edges of the data that is selected to be transformed. The mean SCL and frequency of SCR's within each sampling timepoint (i.e., T1-T8) were analyzed and extracted also using AcqKnowlege, and a threshold ranging from $0.03 \mu \mathrm{S}-0.05 \mu \mathrm{S}$ was set to ensure valid responses. This threshold range is typically suggested in the literature (e.g., Braithwaite, et al., 2013; Dawson et al., 2007), and any deviations in the signal that do not satisfy these threshold criteria will not be counted as SCR's. HRV was extracted using the Kubios HRV analysis software package (Tarvainen et al., 2014), version 2.2 for Mac OS. The Kubios HRV uses an adaptive QRS detection algorithm to extract HRV from the ECG recording. Within this pre-processing phase, the ECG data is filtered using a bandpass filter to reduce noise and correct for artifacts. Time-domain methods were used to analyze temporal features of HRV. More specifically, the mean value of RR intervals (the time between consecutive heart beats measured in milliseconds) and the mean HR were examined as they are considered to be the most evident features, and are commonly used in the literature (Tarvainen et al., 2014). Threshold-based correction was used to filter the RR interval values and detect and remove any artifacts (e.g., ectopic beats, missed beat detection) without 
distorting the RR interval itself (Tarvainen et al., 2014). The lowest threshold was initially chosen $(0.45 \mathrm{sec}$; will mark any data that is larger/smaller than $0.45 \mathrm{sec}$ relative to the average and will replace the values using interpolation) to avoid losing too much data. The threshold level was adjusted accordingly for each participant taking into account individual variability and the degree of noise in the signal (i.e., threshold would increase as the amount of noise in the signal increased).

6.3.2. Salivary Cortisol Sampling. A total of 8 salivary samples were taken at predetermined time intervals throughout the experimental session in order analyze stress-reactivity. Specifically, salivary cortisol secretion was collected at baseline (T1), following music exposure (T2), following anticipation (T3), TSST (T4), and recovery (T5-T8) (see Figure 1 below for sampling protocol). Samples were obtained using the passive drool method (considered to be a simple and reliable method; Kudielka et al., 2012) with $2 \mathrm{~mL}$ polypropylene cryovials (Salimetrics@) and collection aids to help participants guide their saliva into the cryovials. Participants were instructed to tilt their head forward in order to initiate saliva production and pooling under the tongue. Additionally, participants were instructed to avoid aggressively spitting into the tube, as that would create foam and compromise the quality of the sample. All saliva samples collected were placed on ice for the duration of the study, and were stored in an in-house commercial freezer at a consistent temperature of $-70^{\circ} \mathrm{C}$ until subsequent analyses were conducted.

Samples were analyzed in-house using Salimetrics@ salivary cortisol enzyme competitive immunoassay standard 96-well plate assay kit to extract cortisol from each sample. Prior to analyses, all saliva samples were centrifuged for 15 minutes at 3,000rpm in order to separate any mucins in the saliva. Following incubation and washing, the unbound cortisol is removed. The 
bound cortisol that remains is measured by the reaction between the horseradish peroxidase enzyme to the substrate solution. Once the reaction is stopped, each plate was placed in a BioTek Eon ${ }^{\mathrm{TM}}$ high performance microplate reader (heated to room temperature, $20^{\circ} \mathrm{C}$ ), using Gen5 Software within 10 minutes to reliably detect the concentration of cortisol in each sample. The optical density of each plate was read at both 450 and $490 \mathrm{~nm}$. All samples were assayed in analyses. The intra-assay coefficient of variance (CV) was below $15 \%$ for each sample, as per protocol outlined by Salimetrics. The inter-assay variability was $13.8 \%$, and the intra-assay variability was $4.7 \%$, which is within the acceptable range outlined by Salimetrics to interpret the analyzed data. Experimental results with inter-assay CV's less than 15\%, and intra-assay CV's less than $10 \%$ are generally acceptable (Kudielka et al., 2012; Schultheiss \& Stanton, 2009). Samples were run in duplicate to control for potential pipetting error, and average concentration values were used in analyses. 


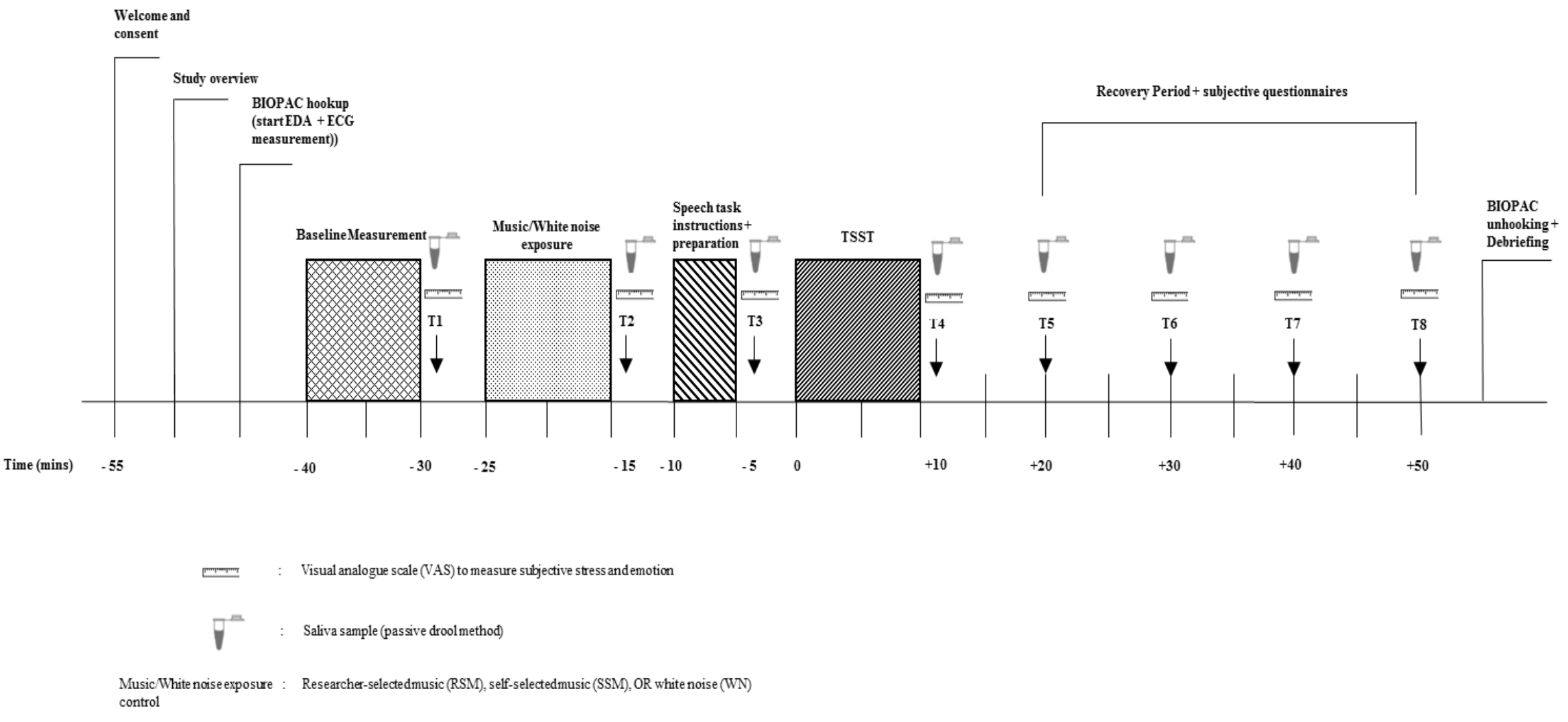

Figure 1. Timeline of study protocol 


\subsubsection{Psychological questionnaires.}

Absorption in Music Scale (AIMS). The AIMS scale is a 34-item questionnaire developed in the Science of Music, Auditory Research and Technology (SMART) Lab at Ryerson University. The absorption factor seeks to examine aspects or qualities of an individual that are associated with the likelihood of becoming absorbed or immersed in music. The willingness or degree to which an individual can become immersed in a situation or activity without distraction is definitive of the term absorption (Tellegen \& Atkinson, 1974). It is expected that individuals who have this ability to become absorbed generally, will also be greatly influenced by music. Previous research has demonstrated a correlation between high absorption and the ability of music to influence mood states of participants (Wild, Kuiken, \& Schopflocher, 1995). Statements included in the scale seek to target abilities to focus attention and ignore other distractions, and enhance cognitive awareness and vivid recollections, all of which are found to be aspects of absorption (e.g., Kreutz et al., 2008), and are suggested to be enhanced by exposure to music. An example statement is "When I listen to music I can get so caught up in it that I don't notice anything." Responses are made on a Likert-type scale ranging from "Strongly Disagree $=1$ " to "Strongly Agree = 5". Cronbach's alpha for the AIMS scale demonstrates good internal consistency overall ( $\alpha$ ranges from .92 to .94; Sandstrom \& Russo, 2013). The AIMS scale was used to assess the moderating effect of music absorption in the relationship between music exposure and stress reactivity.

Depression, Anxiety and Stress Scales (DASS-21). The DASS-21 (Henry \& Crawford, 2005) consists of three 7-item self-report scales that measure depression (DASS-D), anxiety (DASS-A), and stress (DASS-S) separately on a 4-point scale (0-3), where $0=$ "did not apply to me at all", and $3=$ "applied to me very much, or most of the time". Participants are instructed to 
circle a number in the given range, which corresponds to how much each statement has applied to them over the past week. Example items are "I felt I was close to panic" (DASS-A), "I tended to overreact to situations (DASS-S)", and "I felt that I had nothing to look forward to" (DASSD).

In a large non-clinical sample, the reliability of the total scale was high with Cronbach's $\alpha$ at $.93(\mathrm{CI}=.93-.94 ;$ Henry \& Crawford, 2005). The reliability of each sub-scale was also estimated for the depression scale, with $\alpha .88(95 \% \mathrm{CI}=.87-.89)$, the anxiety scale, with $\alpha .82$ $(95 \% \mathrm{CI}=.80-.83)$, and the stress scale, with $\alpha .90(95 \% \mathrm{CI}=.89-.91$; Henry \& Crawford, 2005). Further, the DASS-21 shows good convergent and discriminate validity when compared with other validated measures of anxiety and depression (Henry \& Crawford, 2005). This scale also shows strong reliability and validity in older adult samples in each sub-scale (Gloster et al., 2008). Further, this scale has good convergent validity, and has been suggested for use in older populations in lieu of multiple questionnaires (Gloster et al., 2008). Inclusion of the DASS-21 in the current study was to ensure that groups did not differ on self-reported depression, anxiety and stress.

Music Questionnaire. The Music Questionnaire was developed in the Science of Music, Auditory Research, and Technology (SMART) lab (Director: Dr. Frank Russo) at Ryerson and consists of 9-items which assess level of music exposure, preference, experience, importance, and reasons for listening to music. For example, participants are asked, "How important is music to you?" This scale was included in the protocol to gather information regarding the degree of music exposure, to ensure similar experience with music across experimental groups.

Perceived Stress Scale (PSS). The PSS (Cohen, Kamarck \& Mermelstein, 1983; Cohen, et al., 1988) is a brief 10-item self-report scale, which measures the degree to which life 
situations and experiences over the past month are perceived to be stressful. The PSS is the most widely used psychological instrument for measuring the degree of perceived stress in individual's lives. The questions are designed to tap into various aspects of perceived stress, such as the degree of how uncontrollable or unpredictable life events are considered to be. Some example questions include "In the last month, how often have you been able to control irritations in your life?" and "in the last month, how often have you been upset because of something that happened unexpectedly?" Participants are instructed to respond to the questions on this scale by indicating on a scale of $0-4$, where $0=$ "never, $1=$ "almost never", $2=$ "sometimes", 3 = "fairly often", and 4 = "very often", how often they have felt a certain way during the last month.

The PSS demonstrates high reliability overall with $\alpha$ coefficients ranging from $.84, .85$, and .86 in a variety of young adult samples (Cohen, et al., 1983). The PSS will inform the current research by providing a subjective measure of the degree to how the sample generally perceives stressors in their lives, and to ensure that general perceived stress does not differ between groups.

Visual Analogue Self-report stress scale. This visual analogue scale (VAS) was created by the experimenter to measure the perception of stress "at that moment", throughout the study protocol. The VAS is a bipolar line that measures a characteristic across a continuum (measured at precisely $100 \mathrm{~cm}$ as to represent a continuum from $0-100)$, and participants mark a spot on the line which corresponds to their perception of how stressed they are feeling at that moment. For the purpose of this study, four different scales were presented, each using different phrasing that represents the experience of stress: "How much distress do you feel", "how much discomfort do you feel?", "how much excitement do you feel?", and "how much agitation do you feel?" Participant responses were measured with a ruler to determine the numerical value of the mark 
made on the continuum. A similar VAS scale has been administered in past studies using the TSST (e.g., Kirschbaum et al., 1999).

\subsection{Procedure}

Eligible participants were invited to the Institute for Stress and Wellbeing Research at Ryerson University. Given the diurnal pattern of cortisol secretion, all participants were tested in the afternoon between $13 \mathrm{~h}$ to $18 \mathrm{~h}$ to avoid ceiling effect of the cortisol stress response (Pruessner et al., 1997; Kudielka \& Kirschbaum, 2003).

All participants were asked to refrain from smoking, engaging in strenuous physical exercise, and ingesting caffeine or alcoholic products for at least one hour prior to the testing session, which was confirmed upon arrival prior to testing. Following the provision of consent (Appendix C), participants were randomized into one of three experimental conditions: 1) researcher-selected music (RSM); 2) self-selected music (SSM); and white noise (WN). Sampling was stratified by age, to ensure that an equal number of young and older adults were randomly assigned to each of the three study conditions. White noise was chosen as the control condition, to ensure that any observed music effects were not due to exposure to a continuous auditory stimulus. Accordingly, the WN condition served as an auditory stimulation control group, void of music-specific parameters such as tempo, rhythm, timbre, and modality, to determine whether music can improve stress reactivity relative to baseline.

All participants were fitted with a wireless physiological monitor for continuous measurement of skin conductance activity, heart rate, and pulse rate using the BIOPAC system. Participants acclimated to the lab environment during a 10-minute baseline period. During the baseline period, participants were asked to rinse their mouth with water to minimize any particles that may contaminate the saliva samples. To acclimate to the lab, participants were given a 
choice to browse through magazines provided by the researcher (the magazines were chosen to not be emotionally arousing as to not interfere with establishing baseline levels), or to sit quietly and relax.

Following the 10-minute baseline period, the first VAS scale (Appendix D) and saliva sample was obtained (T1). Following baseline measurement, participants were told that they would either be exposed to music (either of their choosing, or the piece selected by the researcher) or would hear some background noise for 10 minutes, depending on the condition in which they were randomized into. In all conditions, and across participants, the auditory stimulus was delivered through SONY (MDR-XD100) Studio Monitor series headphones in order to minimize any background noise in the testing environment that may influence or interfere with the listening experience of the participants. Volume was pre-set at a consistent level across participants (lower than 70dB) in order to avoid loudness of the stimuli influencing the perception of stress. ${ }^{5}$ Participants were instructed to focus on the music/noise, and to "attend to any sounds they heard and ultimately try to relax". Immediately following the music induction phase, a second VAS rating and saliva sample was obtained (T2).

All participants were then subjected to the TSST. The first component of the TSST consists of an anticipation period where participants are told that they will be required to give a 5-minute speech to satisfy the "language component" of the study. They were told that 2 "evaluators" (confederates from our lab) would be present during their speech to analyze the

\footnotetext{
${ }^{5}$ Participants were instructed to not adjust the volume themselves, and if at any point during the experiment, they wanted to adjust it, to inform the researcher. Further, before each participant heard either the music or white noise, a quick volume check was performed to ensure that the pre-set volume was optimal for them. It was noted if any participants needed to have the volume adjusted slightly.

${ }^{6}$ Participants were given a cover story regarding the influence of music on language perception and production. This was done in order to control for any potential differences in physiological reactivity during the study. For instance, if the participant knows beforehand that they will be exposed to an acutely stressful situation, this could change their naturalistic reaction to the situation.
} 
content of their speech. Young adult participants were told to convince the panel of evaluators why he/she is the most qualified candidate for a job in their desired field that they would be applying for after graduation. To increase socially evaluative threat, young participants were also told that their performance on the task would be directly compared to age-matched students from the University of Toronto. Older adult participants were primed with a stereotype ${ }^{7}$ suggesting that memory and language abilities decline with age, and were then instructed to prepare their speech based on how they feel that they have contributed to society throughout their life. The speech content was different in order to maximize the degree of social evaluative threat and relevance to each age-group. All participants were also told that their speech would be video recorded for later analysis of their performance ${ }^{8}$.

Following the 5-minute anticipation period, participants entered a connecting room where the evaluators were already seated wearing white lab coats and holding clip boards to "record" notes. To enhance evaluative threat, evaluators were instructed not to provide any facial feedback, or deviate from the script if the participant attempted to interact during the task. If the participant stopped generating their speech before the 5-minute period was over, evaluators instructed the participant that they "still had some time left, so please continue".

Directly following the 5-minute speech task, one evaluator stopped the participant and provided instruction for the surprise arithmetic task. The participant was instructed to verbally subtract 13 from the number 996 as fast and as accurately as possible, and continue subtracting by multiples of 13 from every correct answer until they reach zero. On every failed attempt (i.e., incorrect generated number), one evaluator would interject and say, "Stop. Start again at 996".

\footnotetext{
${ }^{7}$ A previous study (Levy et al., 2000) showed that priming older adults with negative stereotypes led to increases in skin conductance, blood pressure, and heart rate compared to those primed with positive stereotypes.

${ }^{8}$ The video camera was turned on to record during the TSST, however the recordings were not used for later analyses. The recording is used as a cover story to enhance the evaluative threat perceived by the participants.
} 
Again, the panel of evaluators remained neutral in expression and did not provide the participant will any facial feedback or other behavioural cues during the task. Immediately following the TSST, the participant returned to the first testing room, and an additional VAS scale and saliva sample (T4) was collected.

Following T4, participants were told that they did not have to perform any more tasks, but will need to sit for a 40-minute period and complete a package of self-report questionnaires. The self-report questionnaires included a brief demographic form, the AIMS, Music Questionnaire, DASS-21, and PSS (Appendices E-I respectively). Questionnaires were presented in the same order to all participants to ensure that questionnaires assessing stress and more emotionally charged items (e.g. DASS-21 and PSS) were administered at the end of the recovery period, to ensure post-stress recovery. In addition, the participant was informed that at each 10minute interval, the experimenter would come in to administer a VAS scale and obtain a saliva sample (T5-T8). Sampling cortisol at 10-minute intervals is consistent with past studies assessing acute psychosocial stress (Dickerson \& Kemeny, 2004; Juster et al., 2016).

After questionnaire completion, participants were unhooked from the BIOPAC system and were thoroughly debriefed given the cover story regarding music and language (Appendix J). The true nature of the study was clarified completely, along with the rationale for all measures used in the study. This experimental protocol was approved by the Ryerson Research Ethics Board (REB-2015-149).

\subsection{Statistical Analyses}

6.5.1. Treatment of raw data. All physiological and psychological outcome variables were tested for normality prior to analyses to ensure that they were normally distributed at each of the eight time points (baseline, music induction, anticipation, TSST, and the four recovery 
periods) using the Kolmogorov-Smirnov (K-S) test. Any time-points that were significantly skewed $(p<.05)$ were transformed (SQRT or $\log 10)$ prior to the main analyses.

BioPAC data. Data points identified as outliers within the sample were examined further using the outlier labeling rule (Hoaglin \& Iglewicz, 1987; Tukey, 1977). Sensitivity analyses were conducted, running analyses with and without outliers. To account for missing data points, multiple imputations were conducted. Prior to imputations, Little's MCAR (missing completely at random; Little, 1988) were conducted in order to determine if the missing values were indeed missing at random. Across measurement time points, the percentage of missing values for SCL activity ranged from $13-13.9 \%$, and $13-14.8 \%$ for SCR's. Further, the percentage of missing values for both HRV and HR ranged from .9-11.1\%. All missing data from the aforementioned physiological indices were due to data that was too noisy to process. As data were not missing by chance (i.e., missing because they contained too much artifact), imputations were not conducted on any raw physiological data (SCL, SCR, cortisol, HR, HRV), and thus all analyses were conducted without imputation.

Given the percentage of missing or invalid data in the physiological outcomes SCL, SCR, HR and HRV, a series of one-way ANOVA's were conducted to determine if any differences existed on baseline demographic and/or psychological characteristics (the same as displayed in Table 1) between those that did not provide data, and those included in all analyses. With respect to participants missing SCL and SCR data, a significant difference was found on their AIMS total score compared to those included $F(1,107)=5.89, p=.02$, such that those who were excluded had higher absorption scores. When looking at HR and HRV missing data, years of education differed significantly between excluded participants compared to included, $F(1,107)=$ $7.00, p=.009$, such that excluded participants reported fewer years of education. However, there 
were no other significant differences found between participants who were included versus those excluded on any other psychological measures at baseline (all $p$ 's $>.05$ ). Chi-squared analyses were also conducted to examine any differences in categorical variables at baseline. Excluded participants did not differ from those included on demographic variables of interest (level of education, ethnicity, and perceived SES) at baseline (all p's >.05).

Cortisol. Mean cortisol concentrations at each time-point were $\log 10$ transformed in order to minimize positive skew, which is a common practice in the literature for biospecimens (Adam \& Kumari, 2009; Sephton, Sapolsky, Kraemer, \& Spiegel, 2000). In addition to examining cortisol at different time points over the course of the study protocol, two indices of cortisol production were calculated. Area under the curve with respect to ground $\left(\mathrm{AUC}_{\mathrm{G}}\right)$ represents the total magnitude of cortisol production (i.e., total cortisol output; Fekedulegn et al., 2007), and area under the curve with respect to increase $\left(\mathrm{AUC}_{\mathrm{I}}\right)$ represents cortisol change over time (Fekedulegn et al., 2007; Pruessner, Kirschbaum, Meinschmid, \& Hellhammer, 2003). Both $\mathrm{AUC}_{\mathrm{G}}$ and $\mathrm{AUC}_{\mathrm{I}}$ were calculated using the trapezoid formulas outlined in Pruessner et al. (2013).

Assessing the change in cortisol over time will allow for a reliable comparison of how music can affect stress reactivity at different time points across participants. The only missing values in the cortisol data were observed during the final recovery period in two participants (1.9\% of the sample), which was due to the inability to produce a sufficient amount of saliva.

Self-report. Within the subjective data the percentage of missing data was quite minimal, ranging from $.9 \%$ to $2.7 \%$. The results of Little's MCAR for subjective outcomes are as follows: $\operatorname{AIMS}, \chi^{2}(34)=43.82, p=.12$; DASS-21, $\chi^{2}(120)=137.173, p=.14$; PSS, $\chi^{2}(25)=4.43, p=$ 1.0; and the VAS, $\chi^{2}(58)=29.16, p=.99$. Based on the results, this data was considered missing at random, and thus imputation was employed. 
6.5.2. Hypothesis testing. Analyses of variance (ANOVA) for continuous data and Chisquare analyses for categorical data were computed to determine whether groups differ on baseline characteristics. Due to well-documented gender (Kirschbaum, Wust, \& Hellhammer, 1992; Kudielka \& Kirschbaum, 2005; Liu et al., 2017) and age-related differences (Kudielka, Buske-Kirschbaum, Hellhammer, \& Kirschbaum, 2004; Meaney, 2001) in stress reactivity, the variables "sex" and "age" were entered into all models as a priori covariates.

To test the first study objective, a series of 8 (Time; baseline, music induction, anticipation, after TSST, recovery 1-4) x 2 (Condition; combined SSM+RSM, WN)) mixed analyses of covariance (ANCOVA) were conducted to determine whether music exposure (regardless of selection) before an acute psychosocial stressor decreases physiological stress reactivity as measured by SCL, SCR, cortisol, HR, and HRV, respectively. The aforementioned method of analyses was also conducted to determine if music can inoculate psychological stress reactivity using the subjective (VAS) outcomes of Distress, Excitement, Discomfort, and Agitation. To address the second objective, a series of 8 (Time; baseline, music induction, anticipation, after TSST, recovery 1-4) x 3 (Condition; RSM, SSM, WN) mixed ANCOVAs were conducted to determine whether selection of music determines the "inoculation effectiveness" of music on both physiological and psychological stress reactivity. These two study objectives were also examined using univariate ANCOVAs for $\mathrm{AUC}_{\mathrm{G}}$ and $\mathrm{AUC}_{\mathrm{I}}$ measurement.

To test the third objective, a median split was calculated for the AIMS, creating a new Absorption variable (High Absorption/Low Absorption). This variable was then included in the aforementioned mixed ANCOVA, to assess a Time x Condition x Absorption interaction. The moderation was tested for each physiological and psychological outcome included in the main 
analyses.

Finally, a series of separate sub-group analyses were conducted for age to explore whether young and older adults respond differently to music condition and whether the moderating role of music absorption differs by age. Analyses were conducted using SPSS Version 22. All analyses were considered significant at $p<.05$. 


\section{CHAPTER SEVEN}

\section{Results}

\subsection{Baseline Characteristics of Participants}

A series of one-way ANOVA's were conducted in order to examine potential group differences in sociodemographic characteristics, physiological, and psychological outcomes at baseline. Chi-square tests were conducted for categorical variables. Baseline characteristics of the three groups (i.e., WN, SSM, RSM) are shown in Table 2. The three groups did not differ significantly at baseline on any of the sociodemographic characteristics of age, sex, education, or perceived SES. In addition, the three groups did not differ on any of the physiological measures at baseline. Lastly, the three groups did not differ significantly at baseline on any of the subjective psychological measures (AIMS, DASS-21, PSS, or any VAS time-points).

Table 2. Baseline characteristics of sample by condition

\begin{tabular}{|c|c|c|c|c|}
\hline Variable & $\begin{array}{c}\mathrm{RSM}(\mathrm{N}=37) \\
\text { Mean }(\mathrm{SD}) \\
\text { or } \% \\
\end{array}$ & $\begin{array}{c}\mathrm{SSM}(\mathrm{N}=38) \\
\text { Mean }(\mathrm{SD}) \\
\text { or } \% \\
\end{array}$ & $\begin{array}{c}\text { WN }(\mathrm{N}=33) \\
\text { Mean }(\mathrm{SD}) \\
\text { or } \% \\
\end{array}$ & $p$-value \\
\hline \multicolumn{5}{|l|}{ Demographic Factors } \\
\hline Age (years) & $44.83(21.47)$ & $46.60(23.44)$ & $41.63(21.23)$ & .65 \\
\hline Sex, $N(\%$ female $)$ & $59.5 \%$ & $65.8 \%$ & $66.7 \%$ & .80 \\
\hline Education (years) & $15.88(2.3)$ & $16.42(2.21)$ & $15.75(2.74)$ & .46 \\
\hline Perceived SES $N(\%)$ & & & & .58 \\
\hline Very Low & $5.6 \%$ & $2.6 \%$ & $0 \%$ & \\
\hline Low & $13.9 \%$ & $7(18.4 \%)$ & $24.2 \%$ & \\
\hline Medium & $72.2 \%$ & $24(63.2 \%)$ & $57.6 \%$ & \\
\hline High & $5.6 \%$ & $5(13.2 \%)$ & $18.2 \%$ & \\
\hline Very high & $2.8 \%$ & $2.6 \%$ & $0 \%$ & \\
\hline \multicolumn{5}{|l|}{ Baseline Physiology } \\
\hline \multicolumn{5}{|l|}{ Skin conductance } \\
\hline SCL mean activity ${ }^{¥}$ & $2.73(.86)$ & $2.67(.80)$ & $2.85(.60)$ & .68 \\
\hline SCR count ${ }^{¥}$ & $6.51(1.84)$ & $6.23(1.93)$ & $6.92(1.86)$ & .36 \\
\hline \multicolumn{5}{|l|}{ Cardiovascular output } \\
\hline $\begin{array}{l}\text { HR (beats per } \\
\text { minute) }\end{array}$ & $74.54(12.51)$ & $71.54(9.61)$ & $74.14(9.67)$ & .58 \\
\hline
\end{tabular}


effect of Condition or a significant Time x Condition interaction.

Table 3. Mixed analysis of covariance results: Effect of music exposure (vs. white noise) on stress reactivity

\begin{tabular}{|c|c|c|c|c|}
\hline Model Effect & $d f$ & $F$ & $p$ & $\eta_{p}^{2}$ \\
\hline \multicolumn{5}{|c|}{ Physiological Reactivity } \\
\hline \multicolumn{5}{|c|}{ Skin Conductance Level } \\
\hline Time & 7,581 & 2.17 & .04 & .03 \\
\hline Condition & 1,83 & .70 & .40 & .008 \\
\hline Time $\mathrm{x}$ Condition & 7,581 & .75 & .63 & .009 \\
\hline \multicolumn{5}{|c|}{ Skin Conductance Response } \\
\hline Time & 7,574 & 1.54 & .15 & .02 \\
\hline Condition & 1,82 & .44 & .51 & .005 \\
\hline Time $\mathrm{x}$ Condition & 7,574 & .81 & .58 & .01 \\
\hline \multicolumn{5}{|l|}{ Heart Rate } \\
\hline Time & 7,588 & 27.27 & $<.001$ & .25 \\
\hline Condition & 1,84 & .04 & .85 & .00 \\
\hline Time x Condition & 7,588 & .74 & .64 & .009 \\
\hline \multicolumn{5}{|l|}{ Heart Rate Variability } \\
\hline Time & 7,588 & 26.27 & $<.001$ & .24 \\
\hline Condition & 1,84 & .24 & .63 & .003 \\
\hline Time $\mathrm{x}$ Condition & 7,588 & 1.15 & .33 & .01 \\
\hline \multicolumn{5}{|l|}{ Salivary Cortisol } \\
\hline Time & 7,679 & 5.85 & .002 & .06 \\
\hline Condition & 1,97 & .08 & .78 & .001 \\
\hline Time $\mathrm{x}$ Condition & 7,679 & .82 & .57 & .008 \\
\hline \multicolumn{5}{|c|}{ Psychological Reactivity } \\
\hline \multicolumn{5}{|c|}{ Distress } \\
\hline Time & 7,658 & 27.89 & $<.001$ & .23 \\
\hline Condition & 1,94 & .00 & 1.0 & .00 \\
\hline Time $\mathrm{x}$ Condition & 7,658 & 1.59 & .14 & .02 \\
\hline \multicolumn{5}{|l|}{ Excitement } \\
\hline Time & 7,679 & 4.98 & $<.001$ & .05 \\
\hline Condition & 1,97 & .49 & .49 & .005 \\
\hline Time $\mathrm{x}$ Condition & 7,679 & 68 & .69 & .007 \\
\hline \multicolumn{5}{|l|}{ Discomfort } \\
\hline Time & 7,679 & 14.06 & $<.001$ & .13 \\
\hline Condition & 1,97 & 1.87 & .17 & .02 \\
\hline Time x Condition & 7,679 & .66 & .71 & .007 \\
\hline \multicolumn{5}{|l|}{ Agitation } \\
\hline Time & 7,637 & 12.02 & $<.001$ & .12 \\
\hline Condition & 1,91 & .35 & .55 & .004 \\
\hline Time $\mathrm{x}$ Condition & 7,637 & .45 & .87 & .005 \\
\hline
\end{tabular}




\subsection{Effect of Music Selection on Stress Reactivity}

7.3.1. Physiological outcomes. Results of the individual mixed ANCOVA's, controlling for age and sex, are presented in Table 4. In testing the effect of music selection (RSM versus SSM versus $\mathrm{WN}$ ) on physiological stress reactivity, analyses showed a significant main effect of Time for all physiological indices of stress, with the exception of SCR ( $p=.17)$. Analyses failed to show a significant main effect for Condition or a significant Time $\mathrm{x}$ Condition interaction. Physiological stress response patterns are presented in Figures 2a-2e.

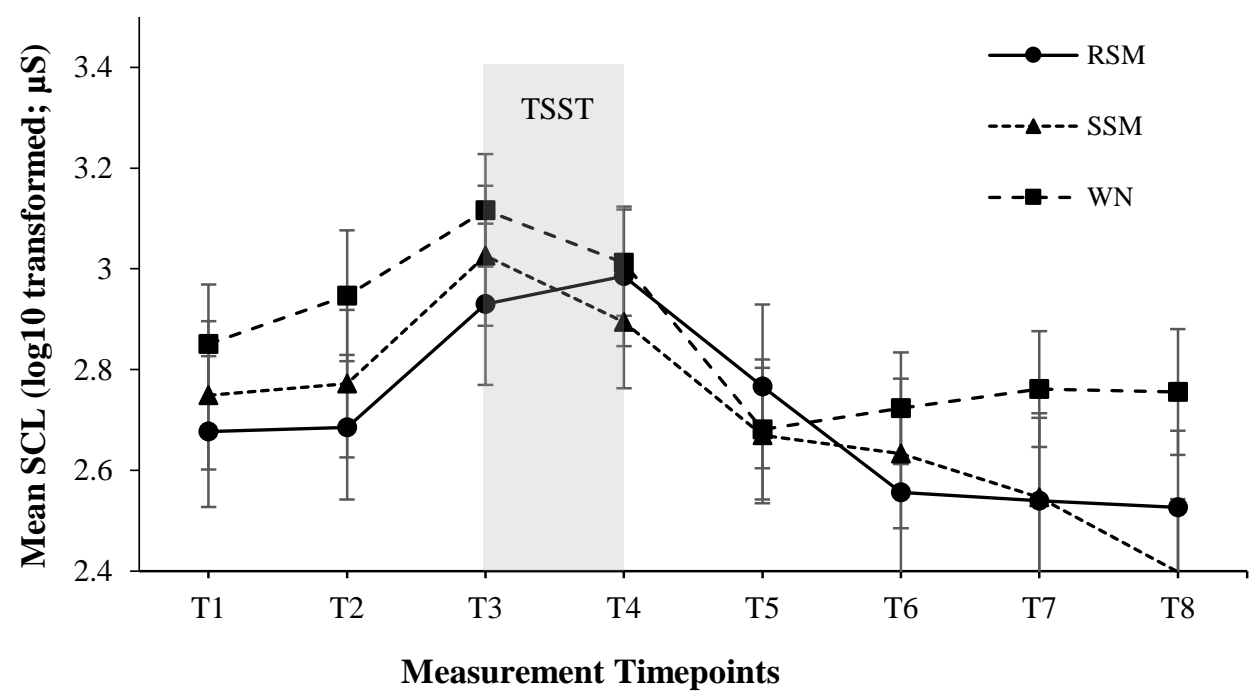

Figure 2a. Mean (SE) Skin Conductance Level (SCL) across time by Condition 


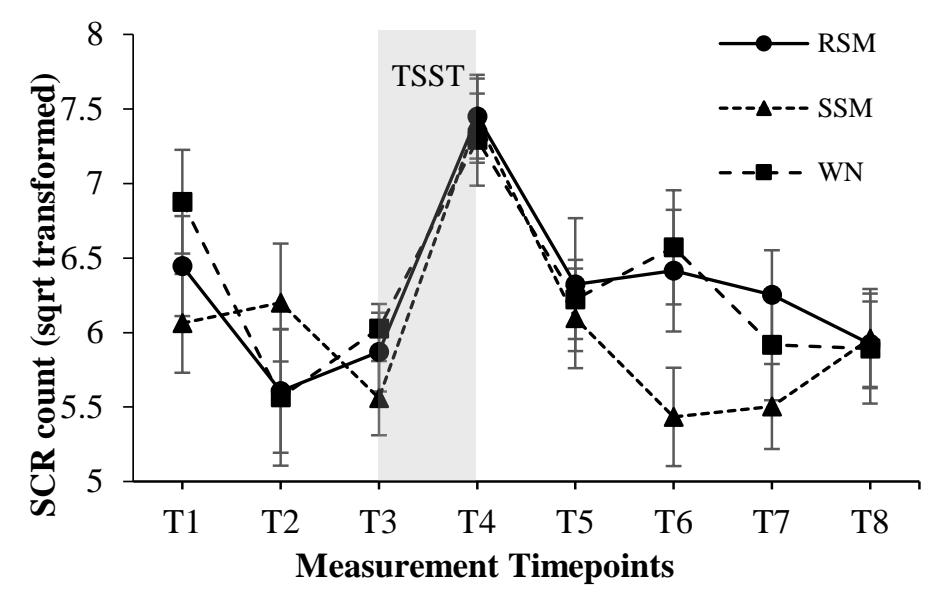

Figure 2b. Mean (SE) Skin Conductance Response (SCR) by condition

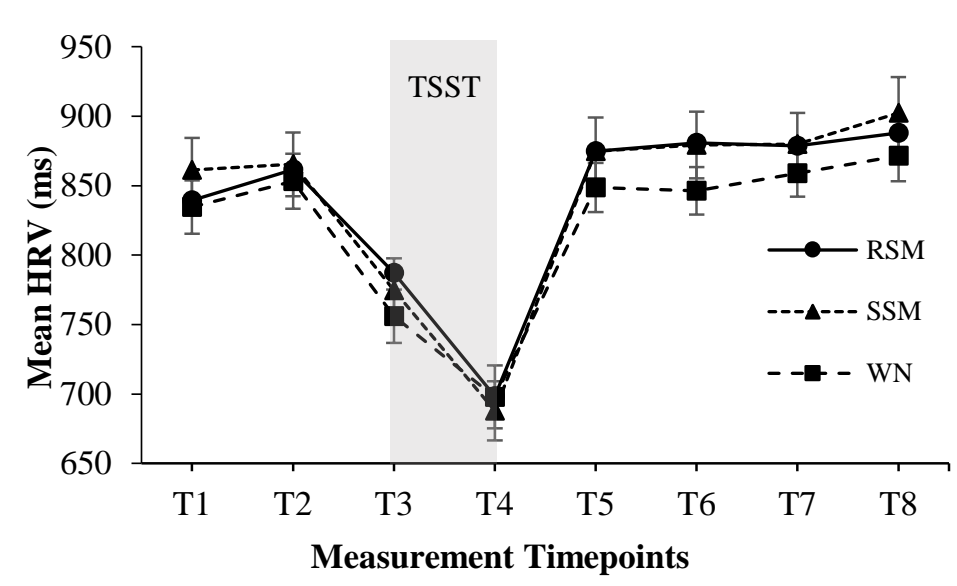

Figure $2 d$. Mean (SE) Heart Rate Variability (HRV) across time by condition

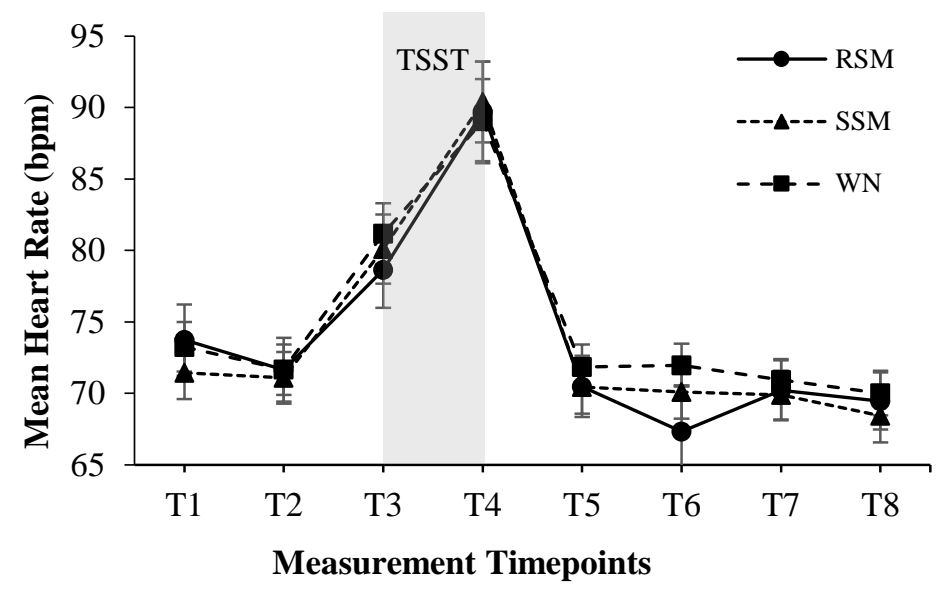

Figure $2 c$. Mean (SE) Heart Rate across time by condition

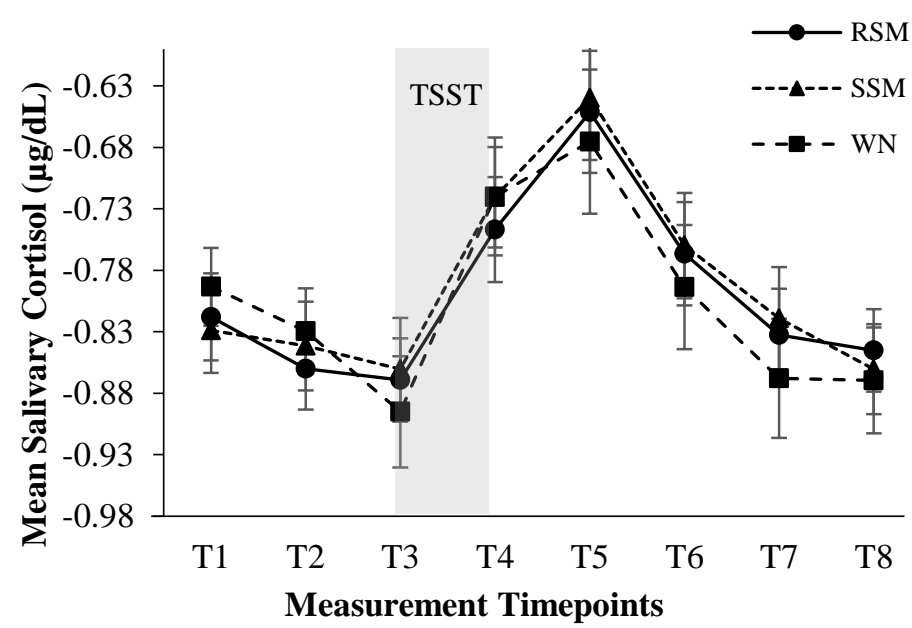

Figure $2 e$. Mean (SE) salivary cortisol (log10 transformed) across time by condition 
Two separate univariate ANCOVA's were also conducted to examine if music condition associated with $\mathrm{AUC}_{\mathrm{G}}$ and $\mathrm{AUC}_{\mathrm{I}}$, controlling for age and sex. Mirroring the aforementioned Mixed ANCOVA analyses, results of the univariate ANCOVA did not show a significant effect of Condition on $\mathrm{AUC}_{\mathrm{G}}, F(2,95)=.16, p=.86$, or $\mathrm{AUC}_{\mathrm{I}}, F(2,95)=.30, p=.75$, suggesting that total cortisol output and increase in cortisol over time was not different between groups.

7.3.2. Subjective Psychological Outcomes. As shown in Table 4, a significant effect of Time was found for all subjective ratings; however, no significant effect of Condition or Time $\mathrm{x}$ Condition interaction was observed. Pattern of subjective response to the TSST is presented in Figure 2f-i. 

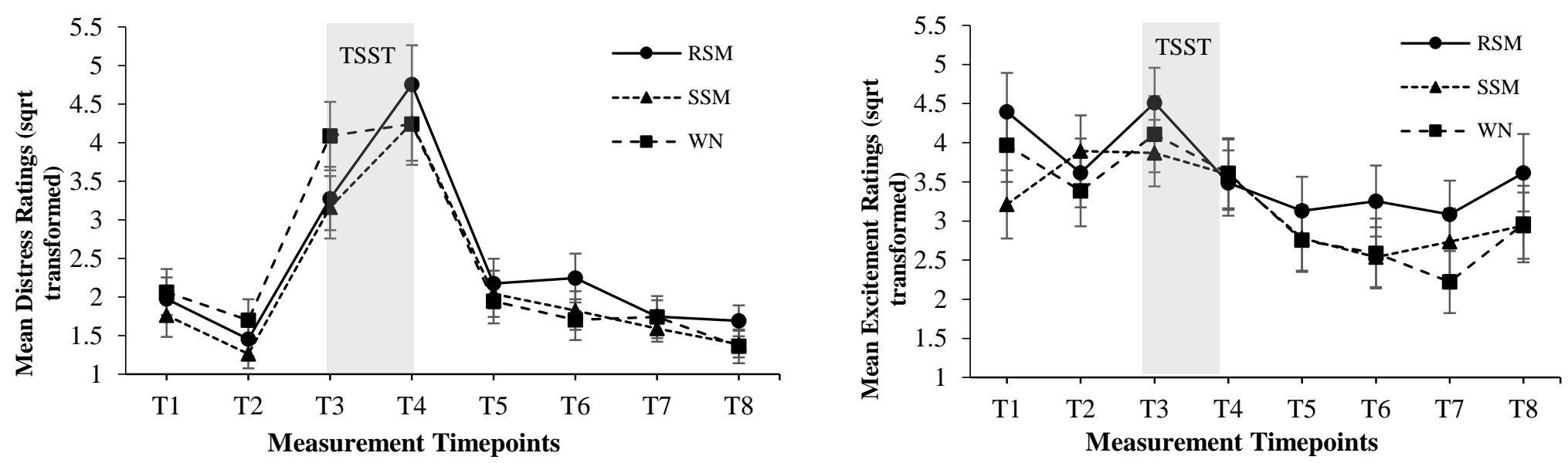

Figure $2 f$. Mean subjective distress across time by condition

Figure $2 g$. Mean subjective excitement across time by condition
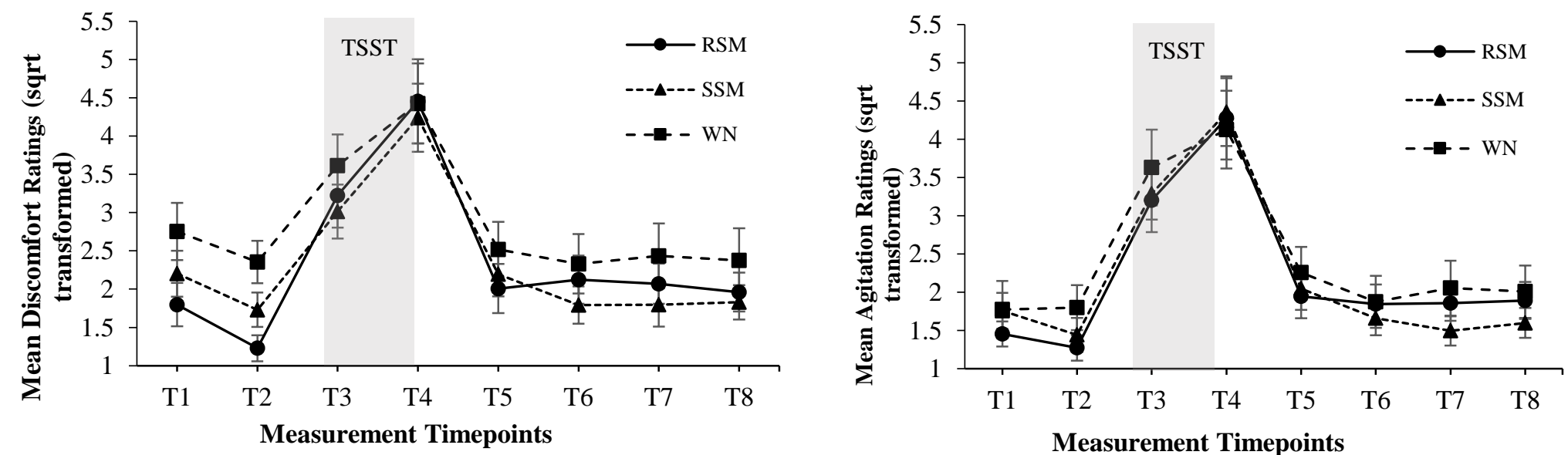

Figure $2 h$. Mean subjective discomfort across time by condition Figure 2i. Mean subjective agitation across time by condition 
Table 4. Mixed analysis of covariance results: Testing the effect of music selection on stress reactivity

\begin{tabular}{|c|c|c|c|c|}
\hline Effect & $d f$ & $F$ & $p$ & $\eta_{p}^{2}$ \\
\hline \multicolumn{5}{|c|}{ Physiological Reactivity } \\
\hline \multicolumn{5}{|c|}{ Skin Conductance Level } \\
\hline Time & 7,574 & 2.30 & .03 & .03 \\
\hline Condition & 2,82 & .35 & .71 & .008 \\
\hline Time x Condition & 14,574 & .63 & .84 & .02 \\
\hline \multicolumn{5}{|c|}{ Skin Conductance Response } \\
\hline Time & 7,567 & 1.48 & .17 & .02 \\
\hline Condition & 2,81 & .69 & .50 & .02 \\
\hline Time $\mathrm{x}$ Condition & 14,567 & 1.10 & .35 & .03 \\
\hline \multicolumn{5}{|l|}{ Heart Rate } \\
\hline Time & 7,581 & 27.83 & $<.001$ & .25 \\
\hline Condition & 2,83 & .02 & .98 & .00 \\
\hline Time x Condition & 14,581 & .89 & .58 & .02 \\
\hline \multicolumn{5}{|l|}{ Heart Rate Variability } \\
\hline Time & 7,581 & 26.93 & $<.001$ & .25 \\
\hline Condition & 2,83 & .13 & .88 & .003 \\
\hline Time $\mathrm{x}$ Condition & 14,581 & 1.06 & .39 & .03 \\
\hline \multicolumn{5}{|l|}{ Salivary Cortisol } \\
\hline Time & 7,672 & 5.60 & .003 & .06 \\
\hline Condition & 2,96 & .05 & .95 & .001 \\
\hline Time $\mathrm{x}$ Condition & 14,672 & .51 & .93 & .01 \\
\hline \multicolumn{5}{|c|}{ Psychological Reactivity } \\
\hline \multicolumn{5}{|c|}{ Distress } \\
\hline Time & 7,651 & 27.97 & $<.001$ & .23 \\
\hline Condition & 2,93 & .32 & .73 & .007 \\
\hline Time $\mathrm{x}$ Condition & 14,651 & .89 & .57 & .02 \\
\hline \multicolumn{5}{|l|}{ Excitement } \\
\hline Time & 7,672 & 4.75 & $<.001$ & .05 \\
\hline Condition & 2,96 & .65 & .53 & .01 \\
\hline Time $\mathrm{x}$ Condition & 14,672 & 1.19 & .28 & .02 \\
\hline \multicolumn{5}{|l|}{ Discomfort } \\
\hline Time & 7,672 & 14.95 & $<.001$ & .14 \\
\hline Condition & 2,96 & .93 & .40 & .02 \\
\hline Time $\mathrm{x}$ Condition & 14,672 & .75 & .72 & .02 \\
\hline \multicolumn{5}{|l|}{ Agitation } \\
\hline Time & 7,630 & 1263 & $<.001$ & .12 \\
\hline Condition & 2,90 & .18 & .84 & .004 \\
\hline Time $\mathrm{x}$ Condition & 14,630 & .46 & .95 & .01 \\
\hline
\end{tabular}


7.3.3. Change in recovery period analyses. Although no significant differences were found between groups for physiological or psychological outcomes over the eight measurement points, visual inspection of the plots may suggest a potential effect of music selection on recovery. As such, a recovery score was calculated using the trapezoid method (see Pruessner et al., 2013), including T5 to T8. A series of univariate ANCOVA's were then conducted post-hoc for each of the physiological and psychological outcomes.

Results of the ANCOVA's examining the physiological variables failed to find significant group differences for physiological outcomes (all $p$ 's $>.05$ ). Among the psychological outcome measured, only discomfort, $F(4,97)=2.58, p=.04, \eta_{p}{ }^{2}=.10$, and agitation, $F(4,97)=$ $3.19, p=.017, \eta_{p}^{2}=.12$, showed significant group differences. Pairwise comparisons for subjective ratings of discomfort showed a tendency for participants in the SSM condition (M $=14.77, S D=21.21)$ to report lower levels of discomfort during recovery compared with participants in the $\mathrm{WN}$ condition $(M=35.39, S D=49.47)$; however, this difference was trending, $p=.058$. With respect to agitation, analyses showed a significant difference between SSM and $\mathrm{WN}(p=.02)$, such that ratings of agitation during recovery were significantly lower in the SSM condition $(M=12.91, S D=14.89)$ compared to the WN condition $(M=33.48, S D=43.87)$.

\subsection{Moderating Effect of Music Absorption}

\subsubsection{Effect Modification of Music Absorption on Physiological Outcomes.}

Mixed ANCOVA evaluating the moderation effect of music absorption found that Absorption did not moderate the effect of music selection on HR or cortisol ( $p$ 's $>.05)$. However, a significant Time $\mathrm{x}$ Condition $\mathrm{x}$ Absorption interaction for HRV, $F(14,560)=1.78, p=.04$, $\eta_{p}{ }^{2}=.04$, and a trending 3-way interaction for $\mathrm{SCR}, F(14,546)=1.65, p=.06, \eta_{p}{ }^{2}=.04$ was found. Subsequent subgroup analyses by High and Low Absorbers for HRV reactivity showed a Time $\mathrm{x}$ 
Condition trend effect in Low Absorbers, $F(14,308)=1.67, p=.06, \eta_{p}{ }^{2}=.07$, but not in High Absorbers, $F(14,231)=1.05, p=.41, \eta_{p}{ }^{2}=.06$. MANCOVA did not show a significant effect of Condition on individual HRV time points $(p>.05)$. Although a 3-way interaction was not found for SCL, a trending 2-way interaction was observed, $F(2,79)=2.94, p=.06, \eta_{p}{ }^{2}=.07$. Specifically, the effect of Condition on SCL was trending for Low Absorbers only, $F(2,42)=2.98, p=.06, \eta_{p}{ }^{2}$ $=.12$, and was not significant for High Absorbers, $F(2,34)=.67, p=.52, \eta_{p}{ }^{2}=.04$. Subsequent analyses showed that in Low Absorbers, SSM displayed lower SCL compared with WN $(M=2.48, S E=.13$ vs $M=2.96, S E=.14$, respectively, $p=.02)$.

To further disentangle the aforementioned 3-way interactions, subgroup analyses were conducted for each Condition, to assess the impact of music absorption on stress reactivity. For HRV, a trending Time $\mathrm{x}$ Absorption effect was found for the WN condition only, F(7, $168)=1.78, p=.09, \eta_{p}{ }^{2}=.07$. Inspection of the graph (Figure 3a), suggests that High Absorbers display greater stress reactivity relative to Low Absorbers, evidenced by lower HRV in response to the TSST. However, subsequent MANCOVA failed to show significant between group differences at each HRV point of measurement ( $p$ 's >.05). Both High and Low Absorbers were comparable with respect to HRV in the RSM and SSM conditions. For SCR, a significant effect of Absorption was found on SCR reactivity in the RSM condition only, $F(7,182)=2.62, p=.01$, $\eta_{p}{ }^{2}=.09$. MANCOVA showed that High Absorbers displayed lower SCR compared with Low Absorbers during recovery at $\mathrm{T} 5(M=5.23, \mathrm{SD}=1.64$ vs. $M=7.11, \mathrm{SD}=2.80)$ and $\mathrm{T} 6(\mathrm{M}=5.71$, $\mathrm{SD}=1.39$ vs. $\mathrm{M}=7.03, \mathrm{SD}=2.70)$; and $\mathrm{T} 8(\mathrm{M}=5.03, \mathrm{SD}=1.91$ vs. $\mathrm{M}=6.69 . \mathrm{SD}=1.85)$. High and Low Absorbers were comparable in the SSM and WN conditions. 


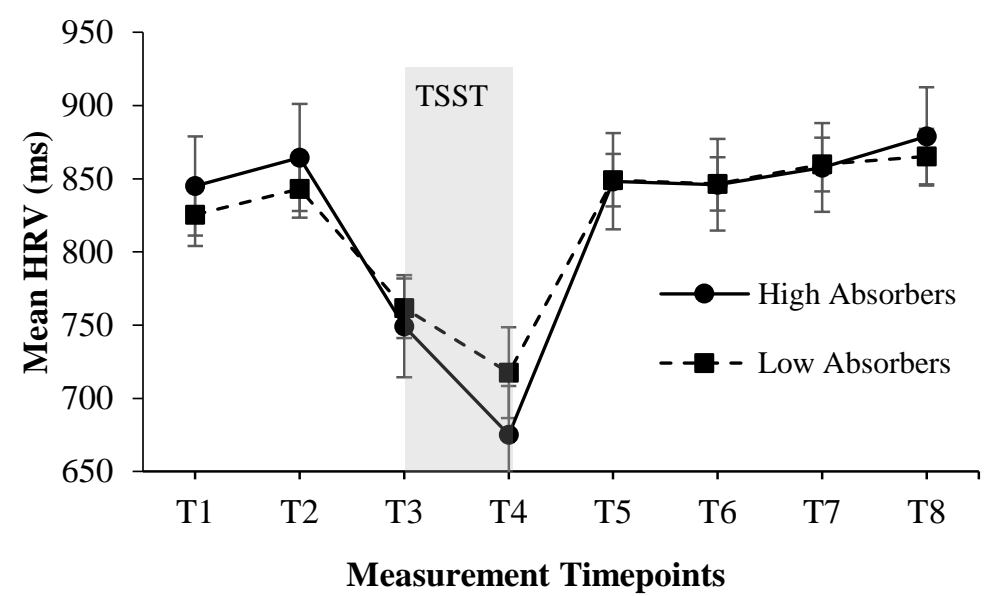

Figure $3 a$. Mean HRV in the WN condition in high and low absorbers across time

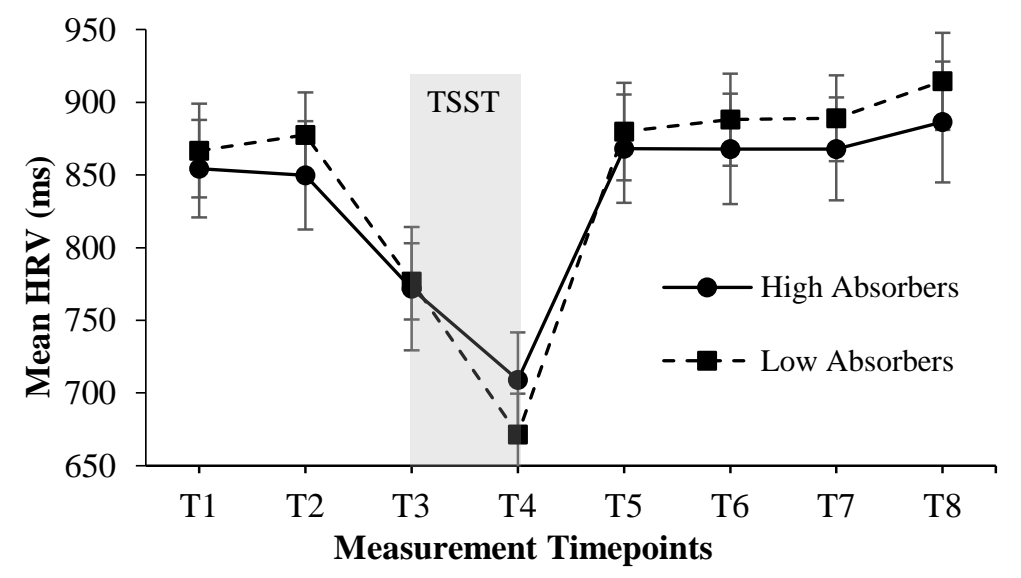

Figure $3 c$. Mean HRV in the SSM condition in high and low absorbers across time

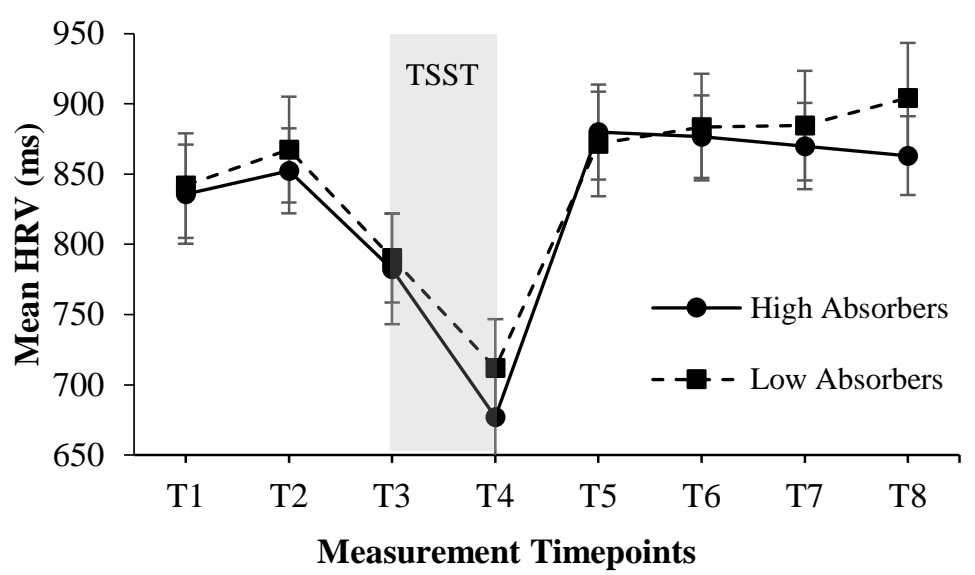

Figure $3 b$. Mean HRV in the RSM condition in high and low absorbers across time. 


\subsubsection{Effect Modification of Music Absorption on Psychological Outcomes.}

Analyses failed to show a significant moderating effect for all subjective reports including Distress, $F(14,630)=.40, p=.97, \eta_{p}{ }^{2}=.009$; Excitement, $F(14,651)=.52, p=.92, \eta_{p}{ }^{2}$ $=.01$; Discomfort, $F(14,651)=.38, p=.98, \eta_{p}{ }^{2}=.008$; and Agitation, $F(14,609)=.55, p=.91, \eta_{p}{ }^{2}$ $=.01$. However, a significant main effect of Absorption was found for Excitement, $F(1,93)=$ 5.23, $\mathrm{p}=.02, \eta_{p}{ }^{2}=.05$, and Agitation, $F(1,87)=5.84, \mathrm{p} .02, \eta_{p}{ }^{2}=.06$. Specifically, High Absorbers reported overall higher levels of Excitement and Agitation compared with Low Absorbers (Figure 4a and 4b).

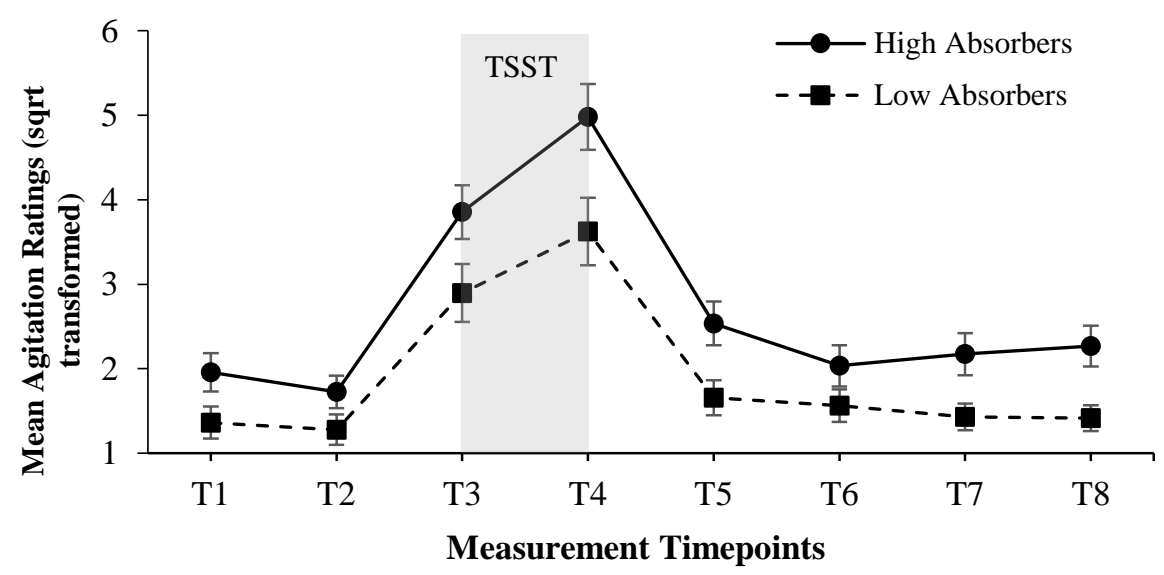

Figure 4a. Mean excitement ratings across time in high and low absorbers

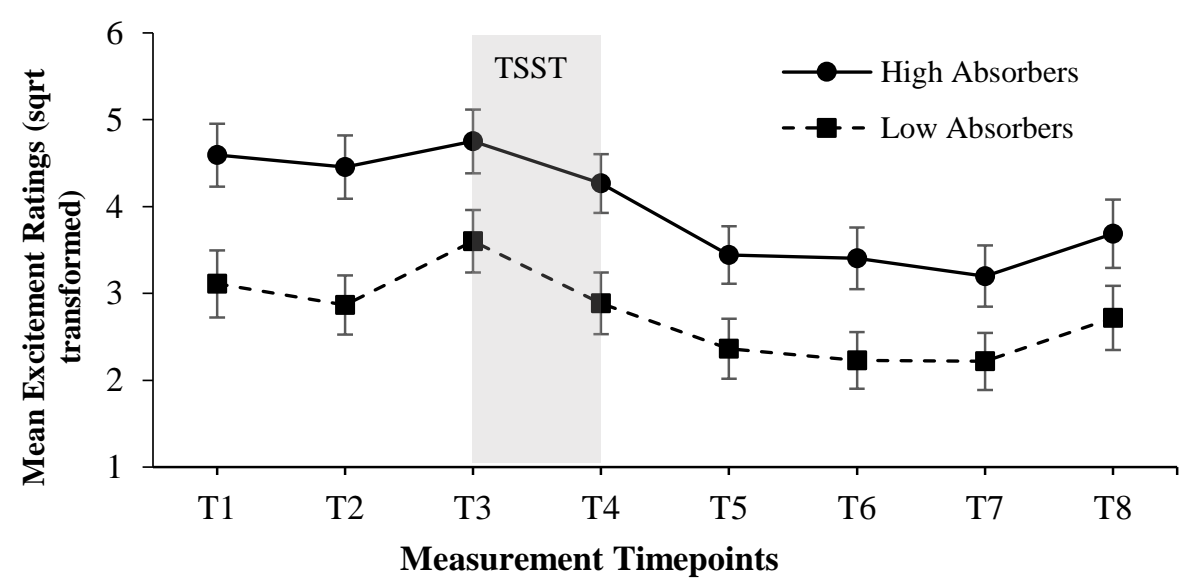

Figure $4 b$. Mean agitation ratings across time in high and low absorbers 


\subsection{Exploratory Age Analyses}

As noted above, to explore the differential effects of music listening and music absorption on stress reactivity in young and older adults, subgroup analyses for Age Group were conducted. To begin, however, the analyses reported below will begin with the analysis on the main effect of Age Group on stress reactivity across physiological and psychological outcomes. All analyses controlled for sex.

\subsubsection{Effect of Age on Stress Reactivity.}

7.5.1.1. Physiological Stress Reactivity. One-way ANOVA revealed significant differences between young and older adults on AIMS scores at baseline $(M=114.77, S E=3.39$ vs. $M=100.73, S E=3.38 ; p=.005)$ respectively, and on PSS scores at baseline $(M=18.11, S E=.90$ vs. $M=10.50, S E=.91 ; p<.001)$ respectively. As such, AIMS and PSS scores were controlled for in the subsequent analyses examining age-related differences in stress reactivity.

Mixed ANCOVAs showed a significant Age x Time effect on cortisol, $F(7,700)=3.29$, $p=.002, \eta_{p}{ }^{2}=.03$, and a trending effect for $\operatorname{SCR}, F(7,588)=1.83, p=.08, \eta_{p}{ }^{2}=.02$. However, with respect to cortisol, subsequent MANCOVA did not reveal group differences at any time point ( $p$ 's >.05). No two-way interactions were found for SCL, HR, or HRV ( $p$ 's >.05). Further, no between groups differences were found for AUCg or AUCi, on cortisol levels ( $p$ ’s >.05).

7.5.1.2. Psychological Stress Reactivity. With respect to psychological outcomes, only ratings of excitement showed significant group differences, $F(1,100)=4.84, p=.03, \eta_{p}{ }^{2}=.05$. More specifically, young adults reported higher levels of excitement compared to older adults $(M=3.73, S D=.27$ vs. $M=2.73, S D=.33$ respectively).

\subsubsection{Effect of Music on Physiological Outcomes in Young and Older Adults.}

7.5.2.1. Skin conductance level in young adults and older adults. In young adults, there 
was no significant effect of music listening (SSM+RSM vs. WN), or music selection (SSM vs. RSM vs. WN) on SCL reactivity $\left(\eta_{p}^{2}=.01\right.$ and partial $\eta_{p}{ }^{2}=.02$, respectively $)$. In older adults, a significant main effect of music listening was found, $F(1,36)=5.44, p=.03, \eta_{p}{ }^{2}=.13$.

Specifically, listening to music (SSM+RSM) resulted in an overall lower SCL $(M=2.50, S E=$ .11) compared with WN $(M=2.88, S E=.13)$. Further, in older adults, the effect of music selection was significant, $F(1,35)=7.76, p=.002, \eta_{p}{ }^{2}=.31$, such that the SSM condition $(M=$ 2.25, $S E=.09)$ displayed a lower SCL compared to RSM, $(M=2.72, S E=.12)$ and compared with WN $(M=2.88, S D=.13)$. RSM and WN did not differ on SCL. See Figure 5.

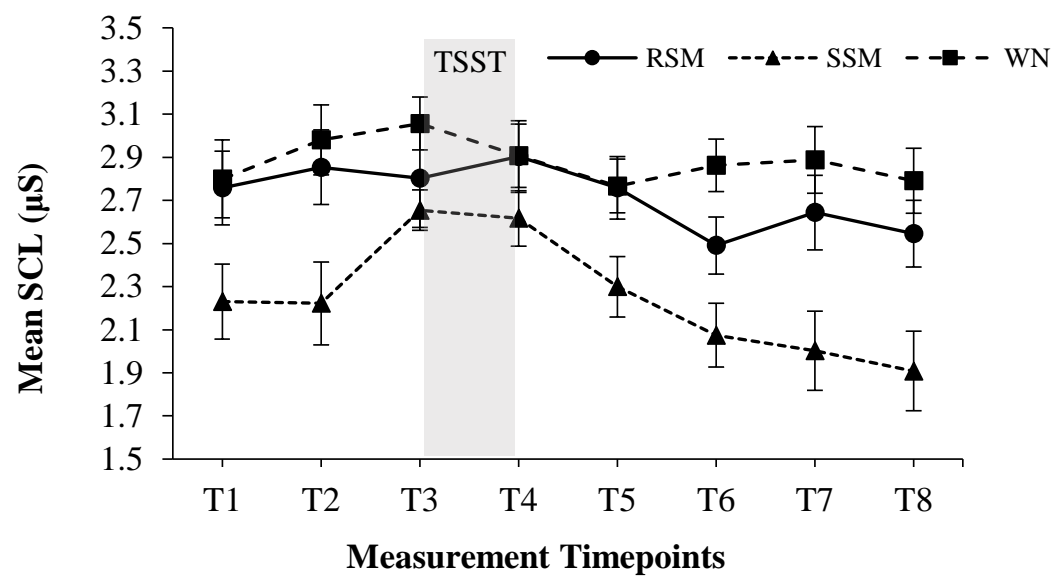

a.

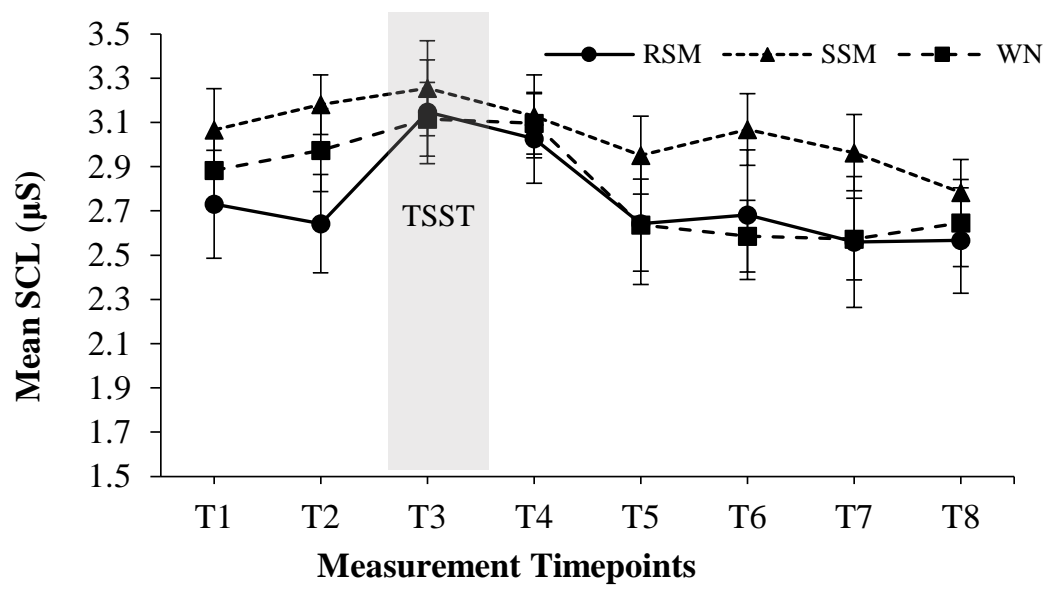

Figure 5. Mean SCL in (a) older adults and (b) young adults following the TSST (T4) and during recovery (T6-T8). Mean SCL scores are (SQRT) transformed. 
7.5.2.2. Skin conductance response in young adults and older adults. In young adults, analyses failed to show a significant effect of music listening (SSM+RSM vs. WN), or music selection (SSM vs. RSM vs. WN) on SCR (partial $\eta_{p}{ }^{2}=.006$ and partial $\eta_{p}{ }^{2}=.03$, respectively). Null findings for the effect of music listening and music selection were similar in older adults (partial $\eta_{p}^{2}=.03$ and partial $\eta_{p}^{2}=.06$, respectively).

7.5.2.3. Heart rate and heart rate variability in young adults and older adults. In young adults, analyses failed to show a significant effect of music listening (SSM+RSM vs WN) or music type (SSM vs. RSM vs. WN) on $\mathrm{HR}\left(\eta_{p}{ }^{2}<.01\right.$ and $\eta_{p}{ }^{2}=.02$, respectively) or $\mathrm{HRV}\left(\eta_{p}{ }^{2}=.01\right.$ and $\eta_{p}{ }^{2}=.03$, respectively). Null results were also found for older adults with respect to the effect of music listening on $\operatorname{HR}\left(\eta_{p}{ }^{2}=.009\right)$ and $\operatorname{HRV}\left(\eta_{p}{ }^{2}=.02\right)$ and the effect of music selection on $\operatorname{HR}\left(\eta_{p}{ }^{2}=.08\right)$ and $\operatorname{HRV}\left(\eta_{p}{ }^{2}=.06\right)$.

7.5.2.4. Cortisol reactivity in young adults and older adults. In young adults, analyses failed to show a significant effect of music listening (SSM+RSM vs. WN), or music selection (SSM vs. RSM vs. WN), on cortisol level $\left(\eta_{p}{ }^{2}=.01\right.$ and $\eta_{p}{ }^{2}=.02$, respectively). Similarly, in older adults, no significant effects were found for music listening $\left(\eta_{p}{ }^{2}=.03\right)$ or music selection $\left(\eta_{p}^{2}\right.$ $=.04)$ on cortisol levels.

\subsubsection{Effect of Music on Subjective Psychological Outcomes in Young and Older adults.}

7.5.3.1. Distress in young adults and older adults. In young adults, analyses failed to show a significant effect of music listening (SSM+RSM vs. WN) or music selection (SSM vs. RSM vs. WN) on subjective ratings of distress $\left(\eta_{p}^{2}=.03\right.$ and $\eta_{p}{ }^{2}=.01$, respectively). Null effects for music listening $\left(\eta_{p}{ }^{2}=.001\right)$ and music selection $\left(\eta_{p}{ }^{2}=.01\right)$ on ratings of distress were also found in older adults. 
7.5.3.2. Excitement in young adults and older adults. In young, analyses showed null findings for both the effect of music listening (SSM+RSM vs. WN), and music selection (SSM vs. RSM vs. WN) on subjective ratings of excitement $\left(\eta_{p}{ }^{2}=.001\right.$, and $\eta_{p}{ }^{2}=.005$, respectively). Similarly, in older adults, analyses failed to show effects of music listening $\left(\eta_{p}{ }^{2}=.02\right)$, or music selection $\left(\eta_{p}^{2}=.05\right)$ on ratings of excitement.

7.5.3.3. Discomfort in young adults and older adults. In young adults, analyses revealed a trend for the effect of music listening (SSM+RSM vs. WN), $F(1,57)=3.44, p=.07, \eta_{p}{ }^{2}=.06$, such that lower levels of discomfort were reported in the music group SSM+RSM, $M=2.72, S E$ $=.20)$ compared with the $\mathrm{WN}$ group $(M=3.38, S E=.29)$. However, analyses failed to show an effect of music selection on rating of discomfort $\left(p>.05, \eta_{p}^{2}=.02\right)$. In older adults, null effects were found for both music listening $\left(\eta_{p}{ }^{2}=.006\right)$ and music selection $\left(\eta_{p}{ }^{2}=.03\right)$ on ratings of discomfort.

7.5.3.4. Agitation in young adults and older adults. In younger adults, analyses failed to show significant effects of music listening (SSM+RSM vs. WN), or music selection (SSM vs. RSM vs. WN) on subjective ratings of agitation $\left(\eta_{p}^{2}=.02\right.$ and $\eta_{p}{ }^{2}=.02$, respectively. Similarly, in older adults, null effects for music listening $\left(\eta_{p}{ }^{2}=.02\right)$ and music selection $\left(\eta_{p}{ }^{2}=.04\right)$ were found for subjective ratings of agitation.

\subsubsection{Effect of music on change in recovery period in young and older adults.}

A series of univariate ANCOVA's were conducted to determine if any differences exist between younger and older adults in physiological and/or psychological outcomes during the recovery phase as a function of music condition. These ANCOVA's were conducted using the same recovery variables (i.e. AUCg from T5 to T8) as used in the main analyses, controlling for sex. 


\subsubsection{Physiological recovery in young adults and older adults. Analyses failed to}

show a main effect of Condition for all physiological recovery outcomes including SCL, $\left(\eta_{p}{ }^{2}=\right.$ $.04) ; \mathrm{SCR},\left(\eta_{p}{ }^{2}=.04\right) ; \mathrm{HR},\left(\eta_{p}^{2}=.005\right) ; \mathrm{HRV},\left(\eta_{p}{ }^{2}=.01\right)$; and Cortisol $\left(\eta_{p}{ }^{2}=.03\right)$ in young adults. In older adults, analyses failed to show significant effects of Condition on recovery measures of SCR, $\left(\eta_{p}{ }^{2}=.09\right)$; HR, $\left(\eta_{p}{ }^{2}=.02\right), \mathrm{HRV},\left(\eta_{p}{ }^{2}=.05\right)$, or cortisol, $\left(\eta_{p}{ }^{2}=.06\right)$. However, a significant main effect of Condition was found for SCL recovery, $F(2,34)=7.99, p=.001, \eta_{p}{ }^{2}=$ .32. Pairwise comparisons show a significant difference between RSM and SSM $(p<.01)$, such that older adults in the RSM condition showed a higher mean SCL, $(M=7.88, S E=.41)$ during recovery compared to the SSM condition, $(M=6.18, S E=.42)$. Further, WN and SSM conditions differed significantly $(p<.01)$, such that a higher SCL was observed in the WN condition, $(M=8.56, S E=.45)$, relative to the SSM condition, $(M=6.18, S E=.42)$.

7.5.4.2. Psychological recovery in young adults and older adults. In young adults, analyses failed to show an effect of Condition on psychological recovery following the TSST, including Distress, $\left(\eta_{p}{ }^{2}=.04\right)$; Excitement, $\left(\eta_{p}{ }^{2}=.004\right)$, but suggested a trend for Discomfort, $F(2,57)=2.46, p=.09, \eta_{p}{ }^{2}=.08$, and Agitation, $F(2,57)=2.78, p=.07, \eta_{p}{ }^{2}=.09$. More specifically, young adults in the RSM condition reported lower discomfort $(M=20.43, S E=$ 8.14) compared to those in the WN condition $(M=45.11, S E=8.48)$; further, young adults in the SSM condition $(M=24.24, S E=7.92)$ showed a tendency $(p=.08)$ to report lower discomfort during recovery compared with the WN condition. Similarly, young adults reported significantly lower levels of agitation during recovery $(p=.02)$ when listening to $\operatorname{SSM}(M=15.69, S E=7.92)$ compared to WN $(M=42.14, S E=8.13)$. In older adults, analyses failed to show a significant effect of Condition on all subjective outcomes including Distress, $\left(\eta_{p}{ }^{2}=.08\right)$; Excitement, $\left(\eta_{p}{ }^{2}=\right.$ $.09)$; Discomfort, $\left(\eta_{p}{ }^{2}=.06\right)$; and Agitation $\left(\eta_{p}{ }^{2}=.06\right)$. 


\subsubsection{Age differences in music absorption as an effect modifier.}

\subsubsection{Music Absorption on physiological outcomes in young adults and older adults.}

In young adults, mixed ANCOVA evaluating the moderation effect of music absorption failed to find a significant three-way interaction for SCL, $F(14,315)=.82, p=.65, \eta_{p}^{2}=.04$; SCR, $F(14,308)=1.54, p=.10, \eta_{p}{ }^{2}=.07 ;$ HR, $F(14,294)=1.42, p=.14, \eta_{p}{ }^{2}=.06 ;$ or Cortisol $F(14,378)$ $=.80, \mathrm{p}=.67, \eta_{p}{ }^{2}=.03$. A trend was found for $\operatorname{HRV}, F(14,294)=1.58, \mathrm{p}=.08, \eta_{p}{ }^{2}=.07$; however, subsequent analyses did not reveal group differences. Similar null findings were found in older adults; SCL, $F(14,224)=.89, p=.57, \eta_{p}{ }^{2}=.05$; SCR, $F(14,224)=1.38, p=.16, \eta_{p}{ }^{2}=.08 ; \mathrm{HR}$, $F(14,252)=.57, p=.89 \eta_{p}{ }^{2}=.03 ; \mathrm{HRV}, F(14,252)=.62, p=.85, \eta_{p}{ }^{2}=.03 ;$ and Cortisol, $F(14$, $266)=.68, p=.80, \eta_{p}{ }^{2}=.03$.

In older adults, a main effect of Absorption was found for $\mathrm{HR}, F(1,36)=5.89, p=.02$, $\eta_{p}{ }^{2}=.14$ and $\mathrm{HRV}, F(1,36)=6.34, p=.02, \eta_{p}{ }^{2}=.15$. Subsequent analyses showed that Low Absorbers displayed higher mean HR $(M=74.87, S E=2.73)$ compared to High Absorbers, $(M$ $=66.93, S E=2.69)(p=.02)$. With respect to HRV, pairwise comparisons showed a significant difference between Low and High Absorbers ( $p=.02)$, such that Low Absorbers $(M=823.07, S E$ = 30.01) displayed lower HRV compared with High Absorbers $(M=913.83, S E=29.65)$, suggesting greater cardiovascular reactivity.

\subsubsection{Music Absorption on subjective psychological outcomes in young and older}

adults. In young adults, mixed ANCOVAs failed to show a significant 3-way interaction for all subjective reports including Distress, $F(14,364)=.74, p=.74, \eta_{p}{ }^{2}=.03$; Excitement, $F(14$, $378)=.77, p=.71, \eta_{p}{ }^{2}=.03$; Discomfort, $F(14,371)=.52, p=.92 \eta_{p}{ }^{2}=.02 ;$ and Agitation, $F(14,357)$ $=.76, p=.72, \eta_{p}^{2}=.03$.

In older adults, mixed ANCOVA failed to show a significant moderation effect of music 
absorption for Distress, $F(14,259)=.64, p=.83, \eta_{p}{ }^{2}=.03$; Excitement $F(14,266)=.88, p=.58$, $\eta_{p}^{2}=.04$; Discomfort $F(14,273)=1.25, p=.24, \eta_{p}{ }^{2}=.06$; and Agitation $F(14,245)=.47, p=.95$, $\eta_{p}{ }^{2}=.03$. A main effect of Absorption was found for Excitement, $F(1,38)=10.89, p<.01$, $\eta_{p}{ }^{2}=.22$. Pairwise comparisons showed a significant difference between High and Low Absorbers $(p<.01)$, such that High Absorbers reported higher levels of excitement compared with Low Absorbers $(M=3.89, S D=1.73$ vs $M=2.07, S D=1.68$, respectively $)$. 


\section{CHAPTER EIGHT}

\section{Discussion}

The experience of stress is arguably a universal phenomenon, which can have negative consequences for health and well-being when experienced chronically. Recent evidence from Statistics Canada shows that 6.7 million people (23\% of the population) aged 15 years and older report moderate or extreme levels of stress on most days (Statistics Canada, 2014). Exposure to stress can lead to endorsement of risky health behaviours in some individuals such as smoking (McKee et al., 2011), increased alcohol consumption, unhealthy eating habits, and a more sedentary lifestyle (Chandola et al., 2008), leaving the individual more vulnerable to negative health outcomes later in life.

Chronic stress exposure and subsequent dysregulation of stress sensitive systems is associated with a myriad of negative health outcomes including, but not limited to, CVD, depression, and metabolic and immune disorders (Herman \& Cullinan, 1997; Lupien et al., 2009; Tsigos \& Chrousos, 2002). While these negative stress-related health outcomes are observed across different ages, older adults are particularly more vulnerable to the effects of chronic stress due to age-related changes in neuronal structures that are sensitive to the effects of stress hormones (Lupien, et al., 2009; Sapolsky et al., 1986). Accordingly, there is a discernible need for the development and assessment of stress reduction techniques or programs that cater to individuals across the lifespan. The present study aimed to investigate the effectiveness of music listening as a technique that may inoculate stress reactivity to a future stressful encounter in healthy young and older adults. Although previous studies have documented music listening as an effective way to facilitate stress recovery (Berbel et al., 2007; Nilsson et al., 2005; Sandstrom \& Russo, 2010; Linneman et al., 2015), this study was novel as it examined the effect of music as an inoculator to future stress reactivity, measured by objective physiological and subjective 
psychological indices of stress, while considering two important potential modifying variables: music preference and music absorption.

Although it is important to design interventions that aim to facilitate recovery from a stressful experience, it is equally important to elucidate techniques that may prevent future stress reactivity, thus minimizing the amount of perturbations in stress-sensitive systems over time. Existing programs such as the Stress Inoculation Training program (SIT; Meichenbaum, 1972; Meichenbaum \& Turk, 1976) fosters this approach, with the rationale that preventing future stress reactivity is more effective and beneficial for the individual than managing the experience of stress as it arises. As such, prevention of heightened physiological arousal to a stressful experience is emphasized rather than facilitating recovery after the stress has already been experienced. Given the adverse effects of chronic stress exposure on cognitive, emotional, and physical wellbeing (Chrousos, 2000; Juster et al., 2010), targeting ways to prevent heightened stress reactivity and subsequent dysregulation of stress-sensitive systems is crucial.

\subsection{Music Listening as a Technique to Inoculate Future Stress Reactivity}

Contrary to the study hypotheses, music listening and music selection were not found to inoculate the stress response in the entire study sample. A few theories may be considered to explain the null findings of this dissertation, including the music stimuli and the stress induction protocol.

Although improvements in the study design (e.g., piloting the RSM piece, including SSM) was thought to promote the alternate hypotheses, these findings are partially in line with the first study to examine the inoculation effect of music on future stress reactivity. As previously mentioned, Thoma et al., (2013) reported the highest concentration level of cortisol to be in the relaxing music group and lowest concentration level of cortisol to be in the rippling 
water group. While this dissertation did not include a "nature sound" group, it is important to discuss the power of natural environmental sounds on human physiology, as it may provide insight into why music did not inoculate stress physiology in the current study.

It is well known that noxious environmental noise can dysregulate stress physiology (Davies, et al., 2005; Evans, Hygge, \& Bullinger, 1995; Lefèvre et al., 2017). Similarly, relaxing sounds of nature have been documented to facilitate faster skin conductance recovery following acute psychological stress, compared to urban sounds (i.e., traffic noise; Alvarsson, Wiens, \& Nilsson, 2010). Nature therapy seeks to maintain and promote health by facilitating relaxation through exposure to natural stimuli including plants, urban green spaces, and natural stimuli in forests (Kaplan, 1995; Ulrich et al., 1991). A popular type of nature therapy called "Shinrinyoku", defined as "taking in the forest atmosphere, or forest bathing" has received increasing attention in the context of relaxation and stress reduction (Tsunetsugu, Park, \& Miyazaki, 2010). Indeed, numerous studies that examine the effects being surrounded by forest, compared with an urban environment, document reductions in salivary cortisol (Park et al., 2007; Tsunetsugu et al., 2007), increases in parasympathetic activity and decreases in sympathetic activity (Lee et al., 2014; Park et al., 2010), and reductions in blood pressure and pulse rate (Park et al., 2008; Park et al., 2009). Further, forest therapy has been shown to boost immune system function by increasing natural killer (NK) cell activity following three days of forest exposure (Li et al., 2007; Li et al., 2008).

However, these studies have been conducted in Eastern cohorts and must be replicated in Western samples. Further, it is plausible that individual differences may exist for preference of nature sounds (e.g. birds vs. ocean vs. rain), which may affect stress physiology. Additional studies are needed in order to directly compare the effects of natural sounds relative to non- 
natural acoustic sounds on endocrine, autonomic, and immune activity in response to stress.

A second consideration regarding the music stimuli is that music listening may only inoculate stress if there is an explicit goal to reduce the stress response. In this dissertation, participants were blind to the true nature of the study and were not told that they were going to experience a psychosocial stressor after listening to the recording. This is typically done in order to avoid influencing any naturalistic physiological or psychological reactions in response to the acute stressor that may invalidate the results. Although some past studies showing a positive effect of music when recovering from psychological stress have not explicitly told participants to use the music to reduce stress (e.g., Knight \& Rickard, 2001; Sandstrom \& Russo, 2010; Sokhadze, 2007), it is likely more intuitive to use music to help recovery when stressed then to use music as an inoculator before stress exposure. Perhaps music is only effective if the individual is cognizant that the goal of music listening is to reduce physiological reactivity to an imminent threat. A recent study by Linnemann et al., (2015) investigated the stress-reducing effects of music listening in university students $(n=55)$, across five days during the school term and five days during the examination period. Participants were asked to record their perceived stress levels and music listening behaviours four times per day during each of the 10 days. Further, a subset of participants $(n=25)$ provided salivary samples for cortisol extraction on 2 days during each of the two 5-day periods. Although music listening was found to reduce the perception of stress, results showed that the lowest level of perceived stress and the lowest concentration of cortisol were observed when participants reported "relaxation" as their objective for music listening, compared with "distraction" as the main objective. This finding suggests that listening to music with the intention to relax is a key ingredient for the effectiveness of music to reduced stress physiology. 
In addition to the music stimuli, the psychosocial stress paradigm employed in this study may have affected the study outcomes. The literature strongly supports the TSST as a robust and reliable method to induce physiological stress reactivity in the laboratory in both young and older adults (Kudielka et al., 2004). Consistent with previous studies, exposure to the TSST in the current sample induced the typical response pattern to stress in all physiological and subjective indices across participants. More specifically, the highest levels of stress were observed when anticipating the stressor, and directly following completion of the arithmetic task, with peaks in cortisol approximately 10-minutes following cessation of the stressor (Gold et al., 2004; Jezova et al., 2004; Kirschbaum et al., 1993). The strength of the TSST lies in its ability to tap into the major determinants of what characterizes something (or someone) to be stressful: novelty, unpredictability, and socio-evaluative threat (Dickerson and Kemeny, 2004). Given that the TSST employed in the current study contains all of these elements, it is plausible that the physiological reactivity induced by the TSST was impermeable by antecedent music listening. Upon reflection, the current TSST employed a rather potent socio-evaluative threat. While the TSST protocol already includes an evaluative component through the nature of giving a speech in front of a rather unsympathetic panel, the story line employed in the current study included a second layer of threat: stereotype threat. In the current study, student participants were told that their performance was being compared to students from the University of Toronto (a "rival" University) and older adults were told that their performance was being compared to young adults (possibly priming an ageing stereotype of older people performing more poorly on the task than a young adult). It may be speculated that using a milder or moderate psychological stressor could have increased the likelihood of observing an inoculation effect of music listening. To support this thought, MacDonald et al (2003) found that music reduced perceptions of pain in 
individuals having minor surgery, but not in those having more major surgery. It is also important to note that previous studies (e.g., Sandstrom \& Russo, 2010, 2013; Knight and Rickard, 2001) used shorter stress-induction protocols, consisting only of an anticipation period.

A final interpretation of the results, which is in line with the null hypothesis, is that music presented before a stressor is not a viable tool to decrease stress reactivity to a future stressor. As presented in the background of this dissertation, studies that employ music induction during (e.g., Fukui \& Yamashita, 2003) or after (e.g., Khalfa et al., 2003) a stressor report attenuated physiological arousal. Interpreting the current study findings may suggest that music listening is not an effective way to prevent stress reactivity to an acute stressor, but rather functions as a valuable tool to facilitate recovery during or following the experience of a stressor. In the present study, participants listened to music for 10 minutes prior to stress exposure and did not listen to music during or after the TSST. It may be suggested that in order to calibrate stress physiology and emotional arousal, it is vital for the presence of music to be maintained until homeostasis is achieved. While neuroscientists have documented neural correlates of music listening and the impact of music listening on physiological parameters (Koelsch, et al., 2006; Menon \& Levitin, 2005; Salimpoor et al., 2009), to the author's knowledge, it remains unclear whether music listening produces residual effects once the music is no longer playing in order to continuously influence stress physiology. As such, it is possible that in order for music to inoculate the stress response, it must be continuously present throughout stress exposure.

It is worthy to note that analyses showed significantly lower ratings of discomfort and agitation in participants who self-selected their music compared with the white noise control group during the recovery period. These observations of mean differences may allow one to speculate that the "intent" to use music to inoculate stress could enhance these group differences. 
As such, additional research is needed to determine the effects of music exposure (before, during, after stress exposure) and intent (informed to use music for stress management or not informed) on stress reactivity.

\subsection{Music Absorption as an Effect Modifier}

In a previous study by Sandstrom \& Russo (2010), it was reported that young adults considered to be high absorbers experienced faster physiological recovery following acute stress induction when listening to peaceful music, compared to those listening to white noise (Sandstrom \& Russo, 2010; discussed in Sandstrom \& Russo, 2013). Stemming from this study, music absorption may be considered an important effect modifier in the context of using music as a stress inoculation tool. Although music absorption did not show significant modifying effects for all outcome variables, music absorption tended to modify the effect of music selection on SCR and significantly moderated the effect of music selection on HRV. However, results did not support the current hypothesis that the inoculation effect of music listening on stress physiology is greater in high music absorbers compared with low music absorbers. Rather, the role of music absorption appears to be more complex, depending on how the analyses are conducted, which renders more questions than answers regarding the construct of music absorption. When examining the association between music listening and stress reactivity in high absorbers and low absorbers separately, music listening seemed to modulate stress reactivity in low music absorbers only, with SSM inoculating SCL reactivity compared with WN.

Disentangling the modifying effect of music absorption further, investigating the effect of music absorption on stress reactivity across condition, results suggested that high music absorption was associated with greater stress reactivity in the WN condition, evidenced by lower HRV compared with low absorbers. However, this difference in HRV is not found in the music conditions. One 
may draw three speculative conclusions from these results: first, high music absorbers are more reactive to stress than low music absorbers under "normal conditions" (i.e. WN condition); second, this differential in stress reactivity is minimized when exposed to music (i.e. no difference observed in HVR in music conditions); and third, the ability to observe a differential inoculation effect of researcher vs. self-selected music is found in low absorbers only as they are generally less reactive to the TSST, and are therefore more likely to respond to the selected music compared with WN. The notion that high music absorbers are more stress reactive is in line with a previous study that examined the association between general trait absorption and stress reactivity in 26 university students (Zachariae, Jorgensen, Bjerring, \& Svendsen, 2000). Specifically, high trait absorbers were found to be more reactive, evidenced by low HRV, than low trait absorbers. In the current study, high music absorbers also reported greater excitement and agitation during the study protocol compared with low music absorbers. These findings may be compared with the study by Hogue et al. (2016), who not only showed that increase in the tendency to be absorbed by music associated with greater tendency to "like" music (thus suggesting greater emotional responsivity to music), but also showed that an increased tendency to be absorbed by music associated with increased reports of general depression, suggesting an association between music absorption and reports of greater negative affect. However, the effect of music absorption on stress reactivity becomes more complex when analyzing age effects; specifically, low absorbers were more stress reactive than high absorbers with respect to cardiovascular reactivity in older adults, which will be discussed shortly.

Based on these complex results, additional analyses are needed to understand the construct of music absorption. A key feature of the "absorption trait' is the ability to focus attention on one particular stimulus, while ignoring other stimuli present in the environment at 
that time (Kruetz et al., 2008). One may then speculate that persons scoring lower on music absorption may not have the ability to block out their surroundings and narrow their attentional focus on the piece of music being played resulting in greater reactivity to the testing environment compared with high music absorbers. However, this hypothesis would only hold true for older adults. Conversely, when interpreting the sample as a whole (i.e. not divided by age), we may also postulate that high music absorption is associated with heightened stress reactivity in the context of an experimental design where persons are not told the true intention of listening to music. As such, it may be that high music absorbers may be more engaged in the experimental task (i.e., TSST), resulting in greater reactivity, or negativity, as evidenced by higher levels of excitation and agitation in high absorbers relative to low absorbers.

\subsection{Age Differences in the Inoculation Effect of Music}

With respect to the study objective, results suggest that older adults may be more responsive to self-selected music, illustrated by the overall lower skin conductance level in older adults listening to self-selected music. A potential explanation for this finding could be related to degree of association with the chosen music. For instance, older adults choosing their own music may have more deep-seeded associations or positive experiences linked to the specific pieces that were chosen. Older adults typically chose music from artists that were popular during their young adulthood (e.g., the Beatles, Leonard Cohen, data not shown), which could also provide them more autobiographical salience with memories or personal experiences associated with that particular time in their lives. It may be speculated that older adults felt more nostalgia, and a heightened sense of comfort while listening to their own music, and in turn could experience lower levels of physiological arousal as a result. Indeed, literature supports nostalgia as an emotional response that can be triggered by music (Janata, Tomic, \& Rakowski, 2007; Juslin et 
al., 2008). While it is possible that young adults also experienced nostalgia when listening to music they selected, it may be that the effect is stronger in older adults. Although speculative and in need of further investigation, this suggestion is in line with the age-related positivity effect (Mather \& Carstensen, 2005) which posits that older adults tend to focus their attention on positive stimuli (as opposed to negative stimuli) when processing information, resulting in more efficient emotion regulation. Accordingly, it may be suggested that when listening to SSM, older adults in the current study became engaged in the positive emotions associated with their own music, and as such, maintained a lower level of physiological arousal both in response to, and when recovering from the TSST. Still, interpretations are highly speculative as they were only observed for skin conductance and did not extend to other physiological and psychological outcomes.

One peculiar finding was that, in contrast to what was observed in the overall sample, with higher absorbers being more reactive to the TSST (evidenced by lower HRV) than low absorbers, subgroup analysis restricted to older adults showed that low absorbers displayed greater reactivity (evidenced by lower HRV and higher HR) compared to high absorbers. Although the subgroup analyses were conducted in a smaller sample size $(n=39)$, increasing the likelihood that this finding may be spurious, it nonetheless deserves a discussion.

According to age-group analyses, older adults were found to score lower on the AIMS than young adults, suggesting that on average, older adults have a low potential to be absorbed by music than young adults. This finding in itself may stem from the psychometric properties of the scale. Existing research using the AIMS scale has been conducted primarily in young adult samples (Hogue et al., 2016) and was validated in relatively young adults with only an approximate $7 \%$ of the sample consisting of adults over the age of 49 (Sandstrom \& Russo, 
2013). Given the high percentage of older adults considered to be low absorbers relative to high absorbers in the current sample, it is plausible to suggest that the questions included in the AIMS scale may not coincide with how older adults conceptualize music absorption. Further, older adults may have different reasons or purpose associated with music listening compared to young adults, which may have influenced their absorption scores.

\subsection{Strengths and Limitations}

Study Strengths. Although the current study failed to support the dissertation hypotheses, a number of strengths in methodology must be noted. First, this study included three experimental groups, allowing for the comparison between self-selected music, experimenterselected music, and non-music auditory stimulation. This design allows for the comparison of music selection and also provides a proper auditory control group that stimulates auditory processes but is not musical in nature. As such, any benefit observed from music listening could not be attributed to auditory stimulation alone. The second study strength was the inclusion of an important individual difference factor that may moderate the impact of music listening on stress reactivity, music absorption. While previous research has examined the associations between absorption and emotional responsiveness to music (e.g., Garrido \& Schubert, 2010; Kreutz et al., 2008; Wild et al., 1995), to the author's knowledge, this is the first study to examine the potential modifying effect of music absorption as measured by the AIMS scale in the context of stress reactivity.

Third, although the stress protocol may have diminished the potential effects of music to modulate stress reactivity, the TSST protocol is a valid and potent psychosocial stressor with robust psychological and physiological effects (Allen et al., 2014; Buske-Kirschbaum et al., 2007; Kirschbaum et al. 1993; Kudielka et al., 2004). As such, there is no question that all 
participants experienced psychosocial stress during the study. Further, this study incorporated a comprehensive assessment of stress reactivity using physiological, endocrine, and subjective outcome measures. Finally, with the majority of previous studies focusing on young adults, this is the first study to include older adults in the study sample, providing an opportunity to explore age differences in psychological and physiological parameters as they relate to music listening and stress reactivity.

Study Limitations. To improve on the study design, a few limitations may also be reflected on. Although the TSST is noted as a study strength due to its robust impact on stress physiology, it may also be considered a main limitation of this study. This protocol was chosen on the basis of its reliability to induce stress, and its frequency of use within the stress literature (Allen et al., 2017; Dickerson \& Kemeny, 2004; Kirschbaum et al., 1993). However, the degree of stress induced by this technique may have limited the potential effect that music can have on inoculating the stress response. Previous studies that have examined the effect of music during recovery have commonly employed more moderate stressors, including a cognitive challenge task, or a brief anticipation period of giving a speech (Knight \& Rickard, 2001; Sandstrom \& Russo, 2010). Accordingly, additional research is needed to assess the current study hypotheses using a more moderate stress protocol.

A second study limitation is decreased statistical power due to loss of data resulting from unstable physiological measurements. Up to $14.8 \%$ of physiological data points were lost due to excessive noise from the BIOPAC system. The noise was often a result of signal issues while recording and was also a result of gross movements of participants (i.e., sneezing, coughing) while the recording was active. While oversampling of younger adults (an additional 16 younger adults recruited above the recommended $n=45$ ) allowed for a correction of $15 \%$ lost data, it was 
more difficult to recruit older adults. Despite attempts to create equal groups for age, there were fewer older adults $(n=46)$ relative to younger adults $(n=61)$ in the study sample. The challenge in recruiting older adult participant in any study that examines stress reactivity is attribution to the large number of HPA-altering medications that are often prescribed to older adults. In recruiting for the study, 26 older adults were deemed ineligible during the screening process, mainly due to medications they were taking that could potentially influence HPA (e.g., antidepressant) or autonomic activity (e.g. beta-blocker). Further, six older adults who were tested were later deemed ineligible for withholding medical information.

Additionally, a median split was used in order to categorize participants into "high" and "low" absorbers from a continuous absorption score, which may have resulted in misclassification of high and low absorbers. A potential solution would be to split the absorption scores into three groups, and then drop the middle group to more confidently compare a "high" and "low" group. However, this method was not possible given the relatively small sample size.

Finally, although this study included a white noise group to control for auditory stimulation, inclusion of a silent control group as a second control would allow for a stronger study design. Indeed, the current study cannot determine whether white noise is as effective as music, and less effective than silence, in reducing the stress response. Although this study originally included a silent control condition, this condition was later eliminated due to feasibility issues surrounding cost.

\subsection{Implications and Future Directions}

This dissertation contributes to the existing body of literature that examines the use of music as a means of stress reduction and provides important direction for future studies. In particular, additional research is needed to further examine the effect of music exposure timing 
on the stress response. A study designed to directly compare the effect of music presented either before, during, or after acute stress induction could provide insight into the most optimal time to present music in order to achieve maximum stress reducing effects.

The intention of music listening to reduce stress reactivity is equally important and was overlooked by the current study. It would have been interesting to include an additional arm in which participants were aware that the objective of listening to music was to calm the body and mind before a challenge. Stress reduction techniques that are assessed in the laboratory, such as progressive muscle relaxation or mindfulness meditation, are commonly entrenched in the intent to reduce stress reactivity. For example, mindfulness meditation training has been shown to decrease subsequent reactivity to the TSST, likely due to the directed use of mindfulness techniques with the intent of minimizing stress reactivity (Hoge et al. 2013). As such, it would be interesting to examine whether music listening with the intent to minimize stress reactivity can effectively inoculate reactivity to a future stressor.

A question that emerges from this research is whether music can be used as a stress reduction tool for stressors of different magnitude. It is postulated that the TSST employed in the current study may have been too robust for music listening to have a clear impact. Investigating the threshold at which point music is not effective to minimize stress physiology is an important question that may have practical and clinical implications. Directly comparing different stressors could help elucidate which stressors of differing magnitudes are most and least conducive to observing an inoculating effect of music. A number of validated laboratory stressors may be directly compared, including the TSST, TSST anticipation only, the cold pressor task, or a mental arithmetic challenge, to name a few.

Although the current study did not find a robust modifying effect for music absorption, 
this individual difference factor needs further evaluation. Little is known about what it is to be a high absorber. In the current study, older adults scored lower on music absorption compared with young adults. There is little research available to help explain this finding. Further, high absorbers displayed physiology that is characteristic of a high stress responder. Does this mean that high music absorbers have a tendency to become absorbed in their environment, good or bad, which can then influence their affective and physiological state? Future studies are not only needed to provide insight into how this trait may facilitate or inhibit music listening as a stress management tool, but are further needed to understand how music absorptive tendencies correlate with other individual difference traits and states.

Given the widespread neural activation that occurs when listening to music (e.g., Blood \& Zatorre, 2001), future research is needed to examine target neural areas that correlate with music listening and specific features of the musical piece. With the development of imaging techniques, such as functional magnetic resonance imaging (fMRI), participants may undergo a stress challenge while sitting in a scanner and listening to music (relative to other music or nonmusic groups). Incorporating imaging techniques would provide greater insight into the underlying mechanisms that promote music as a stress management tool. For example, imaging tools may provide real-time and continuous data on how the brain reacts to stress, followed by music listening, or the reverse. It may also provide greater insight into the effects of music genre and self- vs- researcher selected music.

Finally, given the mixed findings in the literature regarding the effectiveness of music as a stress reduction technique, future research is needed to determine the most effective way to implement music to reduce stress in a multitude of contexts. Given the interconnectedness of the brain and immune, metabolic, and cardiovascular systems in relation to stress, research focusing 
on determining the underlying mechanisms of how music affects these systems in concert is crucial moving forward in order to guide and inform the design of interventions to maximize the stress-reducing effects of music.

\subsection{Conclusions}

Overall, findings from the current study did not support the hypothesis that music listening, especially listening to self-selected music, inoculates the stress response. Although findings suggest a potential inoculation effect of music listening on stress reactivity in older adults, results were not consistent across all stress indices and thus should be interpreted with caution. Still, these findings are interesting in that they suggest that the inoculation effect of music may function differently between young and older adults and thus requires further investigation. Another finding that warrants further investigation is the association between music absorption and stress reactivity. In particular, does a tendency to be absorbed by music increase the likelihood of being stress reactive?

Despite the unexpected findings, the current study contributes to a growing body of literature examining the effects of music on stress reactivity and recovery. The current findings highlight the complexity of this research and a need for additional studies that consider timing of music, the stress paradigm employed in the study, and the inclusion of a more diverse sample other than young adults. This study also provides a starting point to investigating the differential effects of music in older adults, an expanding segment of the Canadian population. 


\section{Telephone Script for Recruiting - TSST Community Members \& Older Adults}

E.g., "I would like to volunteer for your research"

"I heard about your research and would like to learn more about participating"

\section{Older adult calls in, asking about the study.}

You say:

Absolutely, let me tell you a bit about the study.

\section{You are returning a message that was left by an older adult who called in for the study. \\ You say:}

Hello, this is [name] from the Stress and Healthy Aging Research Lab at Ryerson University, returning your call regarding a study we are conducting looking at the effects of music on language perception and production in healthy older adults. Do you have a few minutes to chat?

\section{Calling a participant for the first time:}

You say:

Hello, this is [name] calling from the Stress \& Healthy Aging Lab at Ryerson University. I'm calling because your name is in a list of participants who might be interested in participating in Psychology research at Ryerson. Is this correct?

We are currently conducting a study looking at the effects of music on language and speaking abilities. Do you have a few minutes to chat?

\section{ASK FIRST BEFORE GIVING ANY INFORMATION:}

Have you ever participated in a study at Ryerson before? If so, please provide a brief description of what you did during the study:

If they have participated in the other TSST study ran last semester ( 2 studies currently; Leo in the Aging Lab, or Erika in memory lab), or any other studies involving the TSST, then they DO NOT QUALIFY FOR THIS STUDY!

**We cannot have individuals participating in the TSST more than once because that will remove the novel and stressful aspect of the task $* * *$

\section{General script:}

\section{FOR OLDER ADULTS SAY THIS:}

Research shows that music can significantly impact one's thinking patterns, verbal abilities, and speech perception. However, much of the current research focuses on younger age groups such as children and young adults. The purpose of this study is to investigate how music listening can influence language perception and production in a group of healthy older adults. 


\section{FOR YOUNG COMMUNITY MEMBERS SAY THIS:}

Research shows that music can significantly impact one's thinking patterns, verbal abilities, and speech perception. However, much of the current research focuses on clinical populations and younger age groups such as children. The purpose of this study is to investigate how music listening can influence language perception and production in a group of healthy young adults.

\section{BOTH GROUPS:}

As a volunteer, you would be asked to come to Ryerson on one occasion. The study session, which will be scheduled in the afternoon, will last for approximately two and a half hours. During this twoand-a-half-hour session, you will be asked to do a brief spoken language task that will assess your perception and production of language. Physiological measures (i.e., heart rate) will be recorded continuously during the study. Eight saliva samples will also be taken throughout the study for later analysis. You will also be asked to fill out a few questionnaires that ask about your emotional state (or how you feel about yourself), and your attitudes. Due to the inclusion of physiological measures such as the collection of saliva samples or recording heart rate, we will have to ask you to refrain from smoking, eating, vigorous exercise and ingesting any caffeinated or alcoholic products for at least one hour before the testing session. This aspect is extremely important to the research and we will remind you of it the day before your scheduled session.

Discomfort or frustration may be experienced during the language task. However, the language task is very short and thus any discomfort experienced will be short lived. You may also experience some discomfort when attached to the physiological monitor; however, this is a non-invasive procedure and the equipment is wireless. Every effort will be made to ensure that you are comfortable with placement of the monitor. If you are eligible to participate, will be paid $25 \$$ at the end of the session for your time. If you qualify for the study, you will also be reimbursed $5 \$$ for TTC transit to Ryerson, for a total of $30 \$$.

If you decide to participate, you will be randomly assigned to one of four conditions where you may or may not be exposed to music. It is important for you to know that you will not be able to choose which condition to participate in. Randomization is very important for research.

Note:

If asked about which group they will be assigned to, tell them that they will be randomized and that you do not have that information. MAKE SURE THAT THEY ARE OKAY WITH THIS. Emphasize how important randomization is to ensure that the research is meaningful.

Are you still interested in participating?

And you are willing to be randomized to one of the four conditions??

If yes: Wonderful, before we move forward, I will have to ask you a few demographic and healthrelated questions to determine your eligibility to be included in the study.

It is important to tell you that all information you give me today is confidential. Any information that identifies who you are (e.g. your name, telephone number) will be removed from the data and will be stored in a separate locked filing cabinet. All data kept on the computer will be password protected and held for 7 years after publication of study results. After that, all data will be properly destroyed. This applies to the information you give me today and for any information you give me during the study. 
...[Proceed to phone screening questionnaire]

If no: I understand. Thank you very much for your time and have a wonderful day.

\section{Script Following phone screening questionnaire:}

Person meets criteria: Wonderful! You meet the requirements to participate in this study. Again, all information you have given me today and all information you will provide during the study is confidential. Any information that identifies who you are (e.g. your name, telephone number) will be removed from the data and will be stored in a separate locked filing cabinet. All data kept on the computer will be password protected and held for 7 years after publication of study results. After that, all identifying information and hard copies of all questionnaires will be properly destroyed. Deidentified electronic data will be kept secure indefinitely for training of future students.

BOOK NOW or

BOOK LATER: I will contact you shortly to book your testing session. If you have any questions in the mean time, please do not hesitate to contact me [provide contact info]

Person does not meet criteria: I am sorry to say that you do not meet the eligibility criteria for this specific study. Often in research, we have exclusion criteria, to make sure that our sample is relatively the same on certain characteristics. I am very grateful for your time and the information you provided today.

Again, all information you have given me today is confidential. The reason why we keep the information you gave me today is very important for research - it gives us a description of who we were able to recruit and who we were not able to recruit from the community. May I have your consent to use this confidential phone-screen data for this purpose? Again, your name will not be attached to the data.

Thank you for your time! 


\section{Phone Screening Questionnaire}

Researcher: To determine your eligibility for the study, I will now ask you a few questions about demographic and health information. This confidential information will be securely stored in our database and is restricted to members of the research team. It is important to note that answering "yes" to the following questions does not necessarily mean that you will be considered ineligible for the study. The collection of all demographic and health-related information we ask is important for our recruitment process. Do you consent to moving forward with the screening questions?

**Removed statement reading: "You do not have to answer any questions that you are uncomfortable with; however, you may be excluded based on the information you choose to withhold."

Telephone Number:

\section{DEMOGRAPHIC QUESTIONS}

\begin{tabular}{|l|l|}
\hline Age: & \\
\hline What is your date of birth? & B \\
\hline Biological Sex: & \\
\hline Ethnicity (e.g., Caucasian, Hispanic, etc.): & \\
\hline Country of birth: \\
Country/countries of descent (i.e., where you \\
\hline $\begin{array}{l}\text { Languages you speak: } \\
\text { Mother Tongue: } \\
\text { age did you learn to speak English? }\end{array}$
\end{tabular}


OLDER ADULT PARTICIPANTS:

Are you retired?
No

Yes

If yes, in what year did you retire? 


\section{HEALTH-RELATED QUESTIONS}

\section{Have you ever had any of the following?}

\begin{tabular}{|c|c|}
\hline Vision problems (glasses, operations): & $\begin{array}{l}\text { No } \_ \text {Yes } \\
\text { If yes, specify: } \\
\text { If yes, is your vision corrected to 20/20 with } \\
\text { glasses? } \\
\text { No }\end{array}$ \\
\hline $\begin{array}{l}\text { Hearing problems (weak hearing, hearing } \\
\text { aids): } \\
\text { (EXCLUDE IF HEARING IS NOT } \\
\text { CORRECTED) }\end{array}$ & $\begin{array}{l}\text { No _ Yes } \\
\text { If yes, specify: } \\
\text { Is your hearing corrected to normal with the hearing } \\
\text { aid? } \\
\text { No }\end{array}$ \\
\hline
\end{tabular}




\begin{tabular}{|c|c|}
\hline $\begin{array}{l}\text { Have you ever been diagnosed with a } \\
\text { Psychiatric illness (Such as anxiety, } \\
\text { depression or bipolar disorder) : } \\
\text { (exclude if current diagnosis: Anxiety } \\
\text { Disorder, Major depressive Disorder, } \\
\text { Bipolar Disorder, Psychotic Disorder } \\
\text { (Schizophrenia), Eating Disorder, } \\
\text { Dissociative Disorder, Personality } \\
\text { Disorders) }\end{array}$ & $\begin{array}{l}\text { No _ } \\
\text { If yes, when were you diagnosed? } \\
\quad \text { Year: } \\
\text { If yes, are you currently taking medication to treat } \\
\text { your symptoms? } \\
\text { No __ Yes } \\
\text { If no, then have you ever taken any medication to } \\
\text { treat your symptoms (PROBE ABOUT TIMELINE) }\end{array}$ \\
\hline $\begin{array}{l}\text { Illegal/recreational drug use: } \\
\text { (exclude if dependence) }\end{array}$ & $\begin{array}{l}\text { No _ Yes } \\
\text { If yes, how frequently? } \\
\text { No __ Yes } \\
\text { If yes, how frequently? }\end{array}$ \\
\hline $\begin{array}{l}\text { Diabetes: } \\
\text { (exclude if uncontrolled; CAN BE } \\
\text { CONSIDERED CONTROLLED IF: AIC } \\
\text { levels are normal and exercise regularly } \\
\text { and have balanced diet; probe further for } \\
\text { this information) }\end{array}$ & $\begin{array}{l}\text { No } \\
\text { If yes, when were you diagnosed? } \\
\text { Year: } \\
\text { If yes, is it controlled with medication? } \\
\text { No __ Yes } \\
\text { If yes, which medication? } \\
\text { Name: }\end{array}$ \\
\hline
\end{tabular}




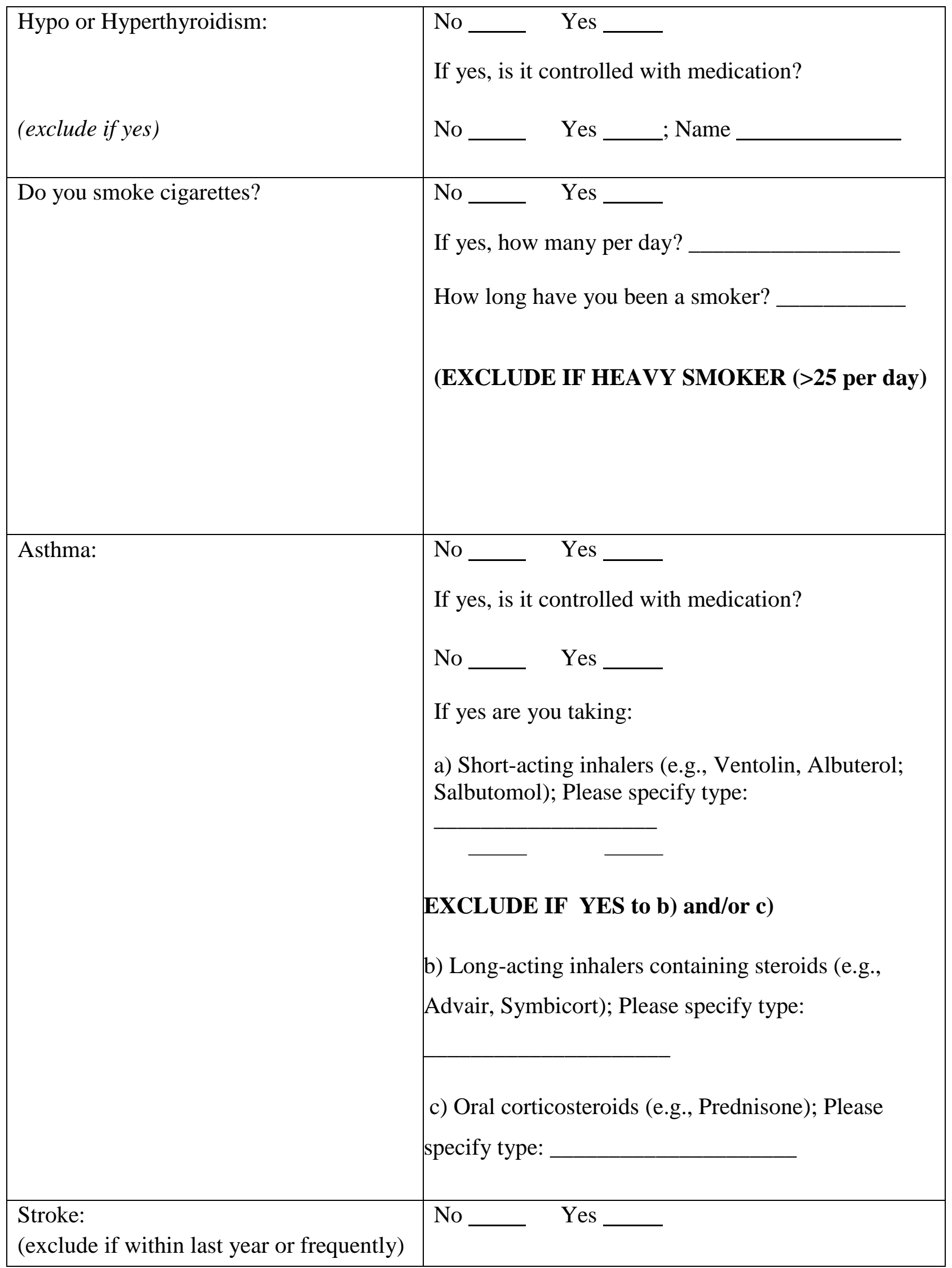




\begin{tabular}{|c|c|}
\hline $\begin{array}{l}\text { Aneurysm: } \\
\text { (exclude if within last year or frequently) }\end{array}$ & No __ Yes \\
\hline $\begin{array}{l}\text { Heart attack: } \\
\text { (exclude if within last year or frequently) }\end{array}$ & No __ Yes \\
\hline $\begin{array}{l}\text { High blood pressure: } \\
(\mathrm{SBP}>=140 ; \mathrm{DBP}>=90) \\
\text { EXCLUDE IF TAKING BETA- } \\
\text { BLOCKERS }\end{array}$ & $\begin{array}{l}\text { No __ Yes __ } \\
\text { If yes, is it controlled with medication? } \\
\text { No __ Yes___ medication name: }\end{array}$ \\
\hline $\begin{array}{l}\text { Neurological disease or neurodegenerative } \\
\text { disease (e.g., Alzheimer's disease): } \\
\text { (exclude if yes) }\end{array}$ & No $\_$Yes \\
\hline $\begin{array}{l}\text { Seizure: } \\
\text { (exclude if not controlled) }\end{array}$ & No __ Yes \\
\hline $\begin{array}{l}\text { Epilepsy: } \\
\text { (exclude if not controlled) }\end{array}$ & No _ Y Yes \\
\hline $\begin{array}{l}\text { Head injury: } \\
\text { (exclude if within last year or traumatic) }\end{array}$ & No __ Yes \\
\hline $\begin{array}{l}\text { Concussion: } \\
\text { (exclude if within last year lost } \\
\text { consciousness) }\end{array}$ & $\begin{array}{l}\text { No }- \text { Yes } \\
\text { If yes, how many? When was the last one? }\end{array}$ \\
\hline $\begin{array}{l}\text { Oral/gum disease: } \\
\text { (EXCLUDE IF SEVERE; WILL } \\
\text { CONTAMINATE SALIVA SAMPLES) }\end{array}$ & $\begin{array}{l}\text { No }- \text { Yes } \\
\text { Type/Description: } \\
\text { Year of diagnosis: }\end{array}$ \\
\hline
\end{tabular}




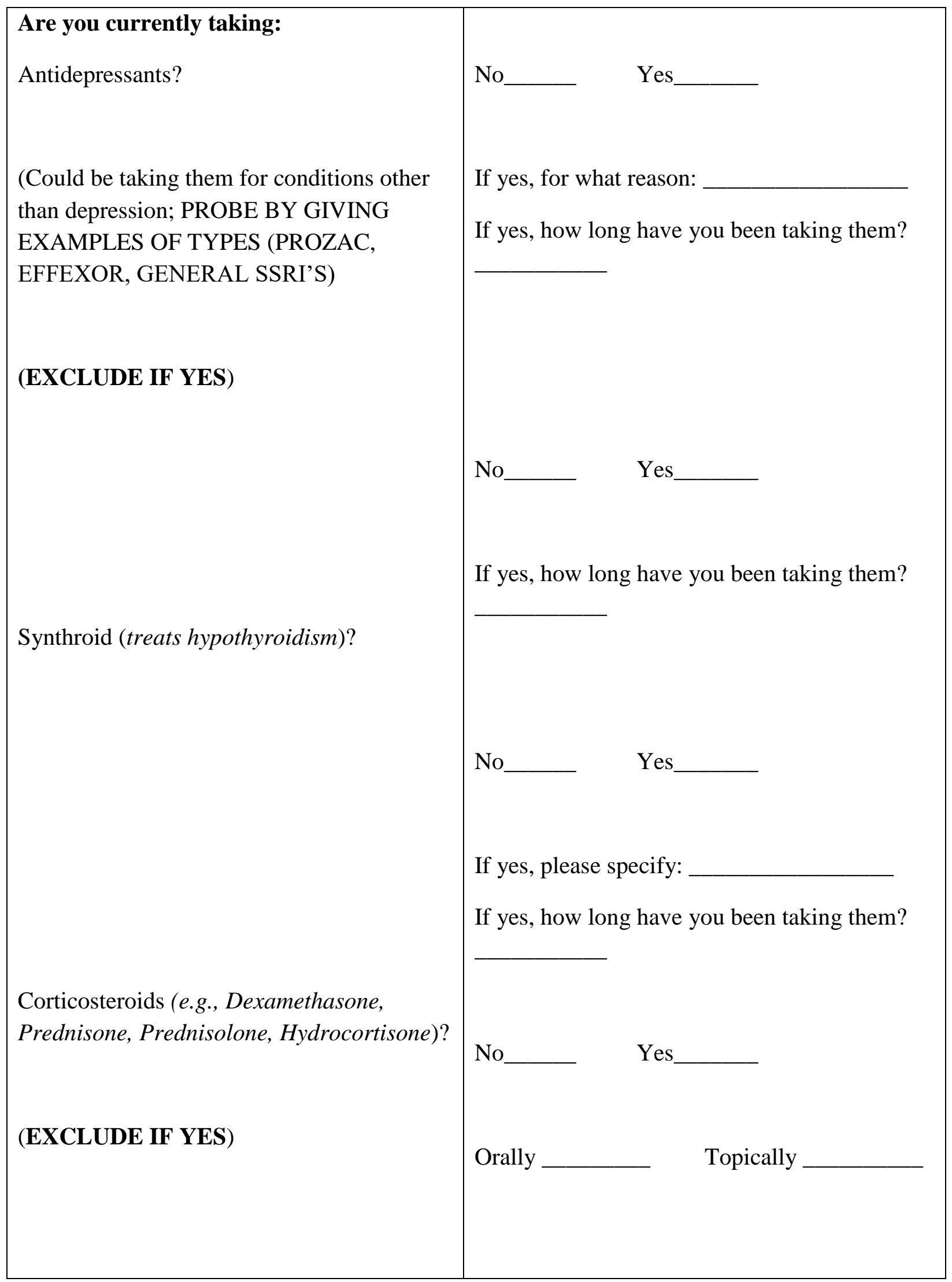




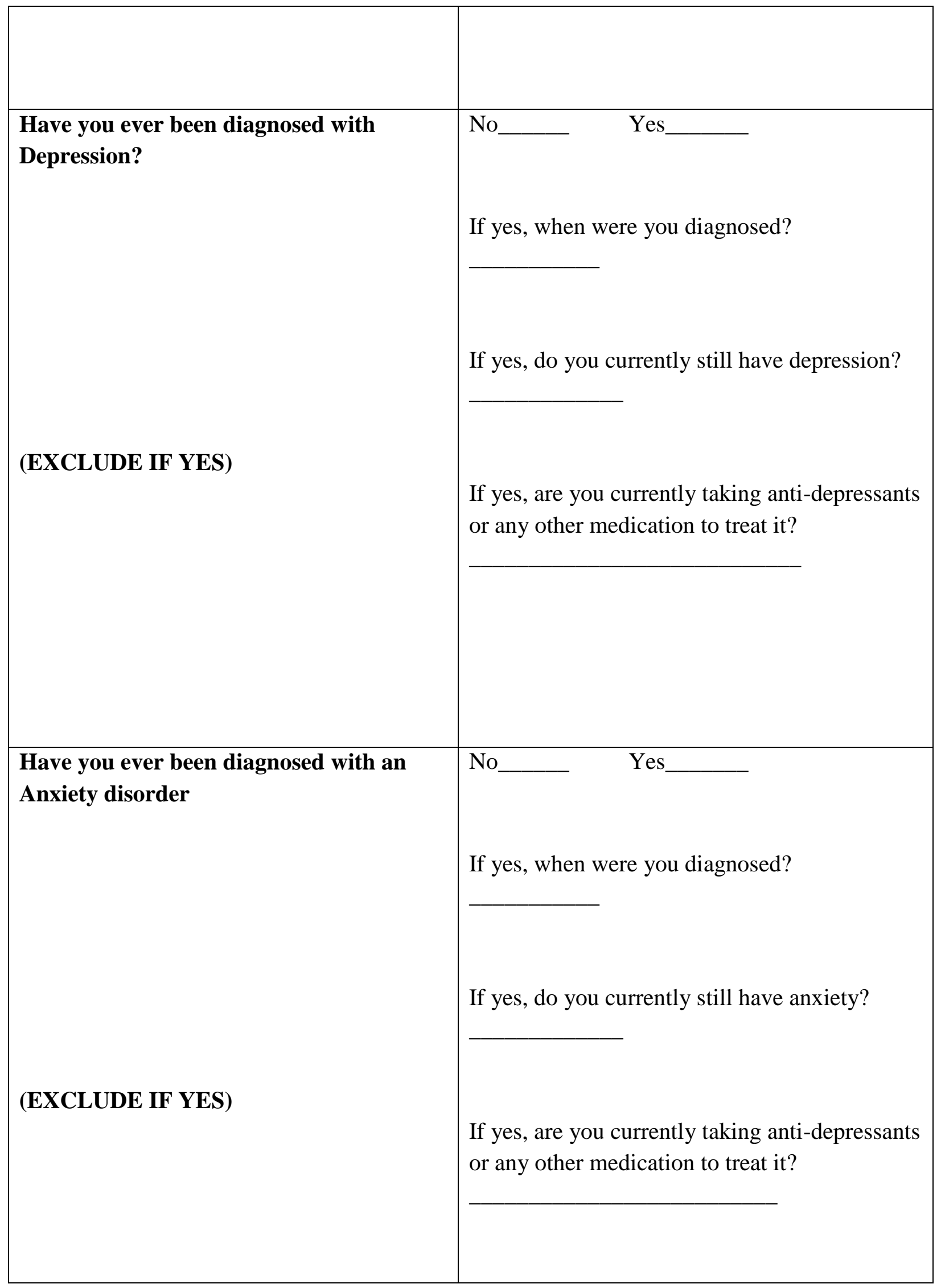




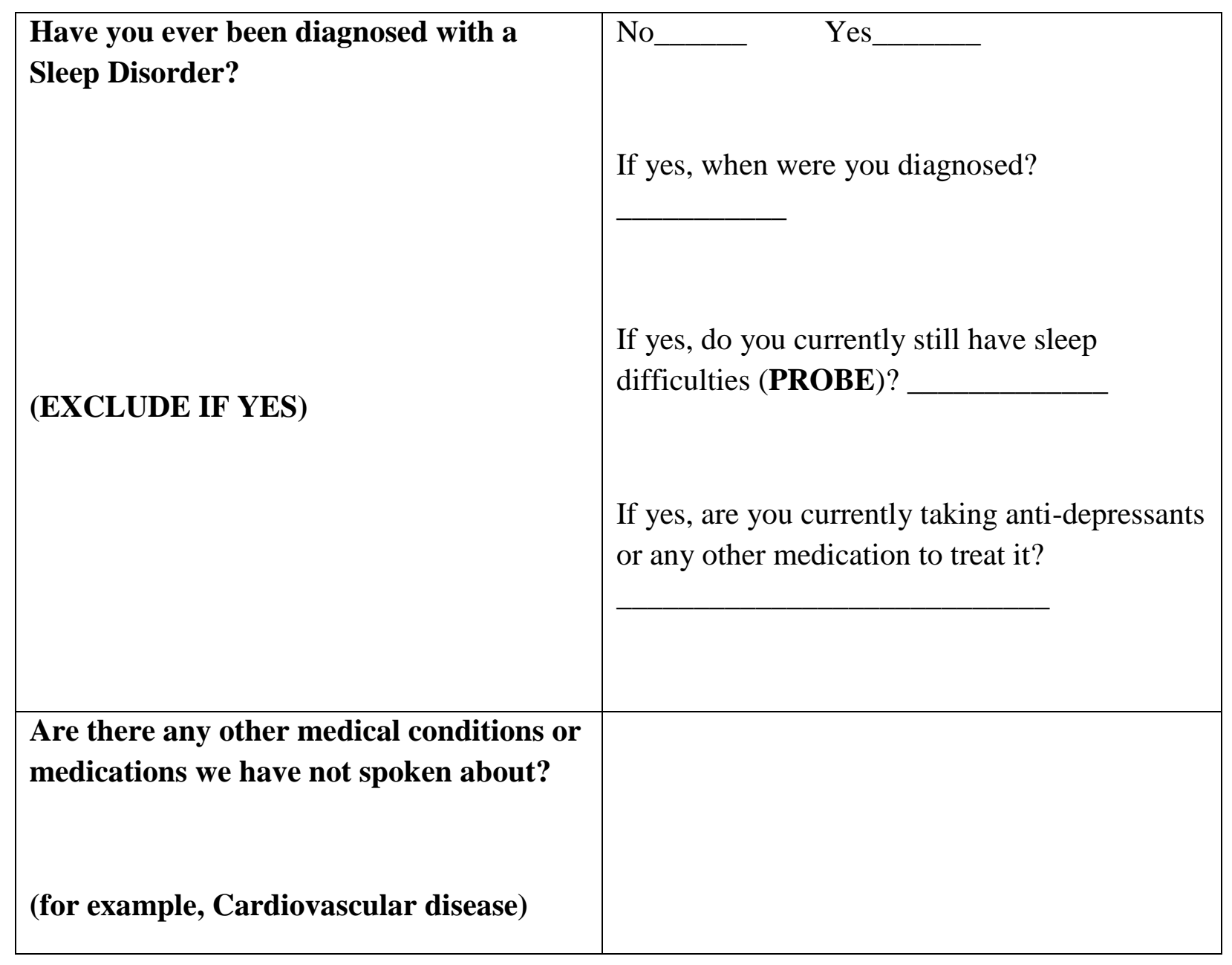

DO YOU CONSENT THAT ALL OF THE INFORMATION THAT YOU HAVE PROVIDED IS TRUE? IT IS VERY IMPORTANT THAT WE HAVE THE CORRECT INFORMATION TO ENSURE NO RISKS IN YOUR PARTICIPATION.

$$
\text { YES NO }
$$

\section{QUESTIONS FOR FEMALE PARTICIPANTS}

\begin{tabular}{|l|l|}
\hline $\begin{array}{l}\text { Are you currently taking oral } \\
\text { contraceptives? }\end{array}$ & No_ Yes \\
& $\begin{array}{l}\text { If yes, when did you start taking them? } \\
\text { If yes, what brand name? }\end{array}$ \\
\hline
\end{tabular}




\begin{tabular}{|c|c|}
\hline $\begin{array}{l}\text { Are you currently taking any other form of } \\
\text { contraceptive that is not administered orally } \\
\text { (i.e., NuvaRing)? }\end{array}$ & $\begin{array}{l}\text { No__ Yes__ } \\
\text { If yes, when did you start taking them? } \\
\text { If yes, what brand name? }\end{array}$ \\
\hline $\begin{array}{l}\text { Are you currently taking any other hormone } \\
\text { replacement medication? }\end{array}$ & $\begin{array}{l}\text { No__ Yes } \\
\text { If yes, when did you start taking them? } \\
\text { If yes, please specify the type and reason for } \\
\text { taking them }\end{array}$ \\
\hline $\begin{array}{l}\text { What is the typical duration of your } \\
\text { menstrual cycle? }\end{array}$ & \\
\hline $\begin{array}{l}\text { Would you consider your cycle to be } \\
\text { irregular or regular? } \\
\text { **If irregular, probe for extent of } \\
\text { irregularity }\end{array}$ & Irregular \\
\hline OLDER ADULT FEMALES: & Yes \\
\hline
\end{tabular}




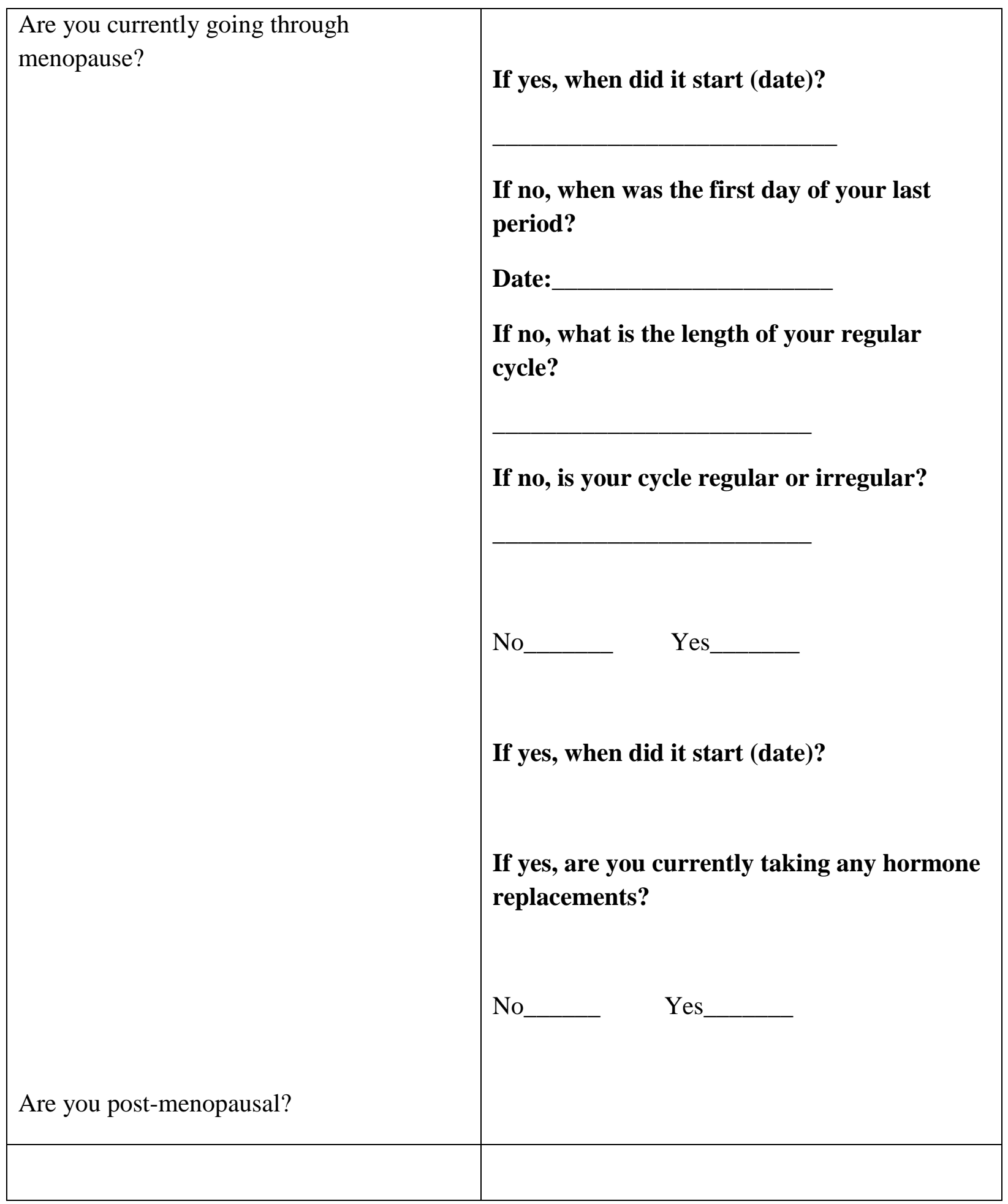


$\underline{\text { Response Bias Questions (taken from the Stress Profile) }}$

Please respond True or False to the following statements:

1) I have never been sick a day in my life $\quad$ True False

2) I have been depressed at least once in my life $\quad$ True False

3) I have never said a bad thing about someone else $\quad$ True False

4) I have never lied in my life $\quad$ True False

5) I always have bad thoughts about other people $\quad$ True False

Do you hereby certify that the above statements are true and correct to the best of your knowledge. Do you understand that a false statement may disqualify you from the study?

\section{YES NO}


Appendix C

Ryerson

University

DEPARTMENT OF PSYCHOLOGY

FACULTY OF ARTS

Consent Agreement

\section{Evaluating the Effect of Music on the Psychology of Language}

You are being asked to participate in a research study. Before you give your consent to be a volunteer, it is important that you read the following information and ask as many questions as necessary to be sure you understand what you will be asked to do.

Investigators: Principal Investigator: Katlyn Peck, MA. Department of Psychology, Ryerson University

Co-investigator: Dr. Alexandra J. Fiocco, PhD., Department of Psychology, Ryerson University

Purpose of the Study: Research shows that exposure to music can significantly impact one's thinking patterns, verbal abilities, and speech perception. The purpose of this study is to investigate how music can influence language perception and production. A total of 80 healthy adults aged 17-35 and 60-80 years will be asked to participate in this research. Potential participants will go through a screening process in order to determine eligibility to participate in the study.

Description of the Study: If you decide to participate in this research, you will be asked to do the following: upon arrival, you will be randomly assigned to one of four conditions where you may or may not listen to music. You will be asked to perform a verbal language task while your blood pressure, heart rate, and other physiological measures will be continuously recorded. This task will be video recorded for scoring purposes only. Saliva samples will be taken for later analysis of hormones. You will also complete subjective measures assessing your current emotional state, personality, and attitudes.

This study will take place in the Psychology Research and Training Centre (PRTC) located at 105 Bond Street. It will take approximately 120 minutes to complete.

What is Experimental in this Study: None of the procedures or questionnaires used in this study are experimental in nature, in the sense that they have all been used previously by other researchers and found to be useful procedures and questionnaires. From a technical or procedural point of view, part of this study is considered "experimental" because by following the procedure described above, the study examines the impact of one variable (called the "independent variable") on other variables (called "dependent variables"). You will be given more information about the independent and dependent variables in this study at the end of today's session.

Risks Associated with Participation: Potential risk associated with participating in this study is minimal and is no greater than what you may experience in your everyday life. Discomfort or 
frustration may be experienced if exposed to undesirable music (e.g. not everyone enjoys classical music) or during the language task. However, music exposure and the language task are very short and thus any discomfort experienced will be short lived. You may also experience some discomfort when attached to the physiological monitor; however, this is a non-invasive procedure and the equipment is wireless. Every effort will be made to ensure that you are comfortable with placement of the monitor. Finally, discomfort may arise from completing psychological questionnaires that ask about your emotions and attitudes. However, you are free to skip any questions that may cause you distress. You are also free to discontinue your participation in the study at any point. As an additional support, the on-call clinicians at Ryerson University's Centre for Student Development and Counseling are available for telephone (416-979-5291) or in-person counsel between 9am and 4:45pm, Monday-Friday.

Benefits Associated with Participation: We anticipate that you will benefit from this study by learning about how psychology research is conducted, and you may also gain a better understanding of research in music and language psychology. When the session is over, we will describe the purpose and hypotheses in the study to you in more detail. Also, once we have analyzed the data (toward the end of the school year), you are welcome to contact the researchers via email to view the results.

Privacy and Confidentiality: All information gathered, including your name, video recordings, and any identifying information will remain confidential. Identifying information, such as your name, will not be attached to any data we collect from you. Instead, we will use a numeric ID number to identify your data. Any documents that contain identifying information, such as this consent form, will be kept in a securely locked cabinet separate from the study data. Hard copies of the data (e.g., questionnaires) will be stored in a secure locked cabinet and all electronic data will be kept in a password-protected file that only the researchers will have access to. Only group summaries (i.e. aggregate data) will be reported for Katlyn Peck's dissertation and any subsequent publications and presentations. The consent form (if you decide to participate) that contains your identifying information, hard copies of the data (e.g. questionnaires), and saliva samples will be kept for at least 5 years after study completion, after which all hard copy data and samples will be destroyed. De-identified electronic data will be kept indefinitely for training of future students.

\section{Incentive to participate:}

Participants will be compensated $\$ 30$ in total. This amount will compensate for approximately 2 hours of participation and cost of time and travel to and from Ryerson University.

Data storage: Data will be securely stored in the Stress and Healthy Aging Research (StAR) Lab ( $1^{\text {st }}$ floor, 105 Bond Street). All data collected will be password protected and encrypted to ensure confidentiality. Identifying information (your name/signature) will only appear on the consent form, which will be stored in a locked cabinet in Dr. Fiocco's office. For all other aspects of the study (i.e. participant age, gender, study data), a unique numeric ID will be assigned for each participant. Names and ID numbers will not be matched, and will be stored separately. All identifying information and hard copies of the data will be destroyed (i.e. deleted, shredded) 5 years after study completion. De-identified data will be stored indefinitely in 
password protected electronic format. The saliva samples collected during the study will be stored in an $-80^{\circ} \mathrm{C}$ freezer in the assay lab within the Harry Rosen Research Commons in the Stress Institute here at Ryerson. All saliva samples will be frozen and stored immediately following the study session for later analysis. Samples will be stored up to 5 years following study completion, after which samples will be destroyed. Only the principal investigator, her research assistant, and her supervisor will have access to the samples.

\section{Voluntary Nature of Participation:}

Participation in this study is entirely voluntary. Your choice of whether or not to participate will not influence any future relations with Ryerson University or the study investigators. If you decide to participate, you are free to withdraw and cease participation at any time without penalty or loss of benefits to which you are allowed.

At any point during the study, you may refuse to answer any particular question or stop participation altogether. If you decide to stop participating, you may also request that your data not be used for the study analysis. You may choose to complete a "walk-through" in which the researcher will guide you through the entire study without collecting your data.

Dissemination of Results: Data collected and results from this study will contribute to Katlyn Peck's doctoral dissertation. As well, results may be shared with others at scholarly meetings or as part of published papers. However, all information will be presented in aggregate form. That is, none of your individual information will be identifiable in any way. Anonymized data may be provided to other researchers for the purpose of study, or verification of results; any data that is shared will NOT include names or identifying information of ANY participants.

Questions About the Study: If you have any questions about this study, or would like information of group results after study completion, please contact the principal investigator, Katlyn Peck (katlyn.peck@ @sych.ryerson.ca), at 416-979-5000 ext 3233, or her supervisor, Dr. Alexandra J. Fiocco (afiocco@ psych.ryerson.ca), at 416-979-5000 ext. 3008.

This project was reviewed by the Ryerson Research Ethics Board (REB\#2015-149). If you have any questions regarding your rights as a human subject and participant in this study, you may contact the Ryerson University Research Ethics Board for information: Lynn Lavallée, PhD., Research Ethics Board, c/o Office of the Vice President, Research and Innovation, Ryerson University, 350 Victoria Street, Toronto, ON M5B 2K3, 416-979-5000, ext. 4791, or by email: rebchair@ryerson.ca 


\section{Consent Form}

\section{Evaluating the Effect of Music on the Psychology of Language}

\section{Agreement:}

Your signature below indicates that you have read the information in this agreement and have had a chance to ask any questions you have about the study. Your signature also indicates that you agree to be in the study and have been told that you can change your mind and withdraw your consent to participate at any time. You have been told that by signing this consent agreement you are not giving up any of your legal rights.

You consent to being video recorded during the language task, which is for the purpose of scoring only:

Agree

Disagree

You have been told that by signing this consent agreement you are not giving up any of your legal rights.

Name of Participant (please print)

Signature of Participant

Date

Signature of Investigator/Study Coordinator

Date

\section{Dissemination of Results:}

If you agree to participate in the study, are you interested in learning about the results of the study in a final report? If so, please provide your mailing or email address below:

Email

Mailing 


\section{Appendix D}

\section{$\underline{\text { VAS }}$}

1. Please make a vertical mark on the line which best corresponds to how you are feeling right now:

1. How much distress do you feel?

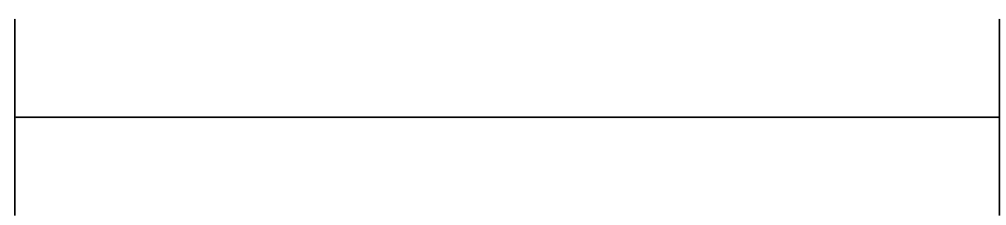

No distress

Worst possible distress

2. How much discomfort do you feel?

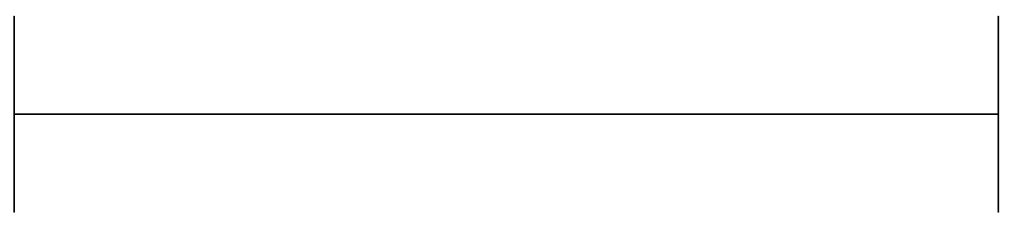

No discomfort

Worst possible discomfort

3. How much excitement do you feel?

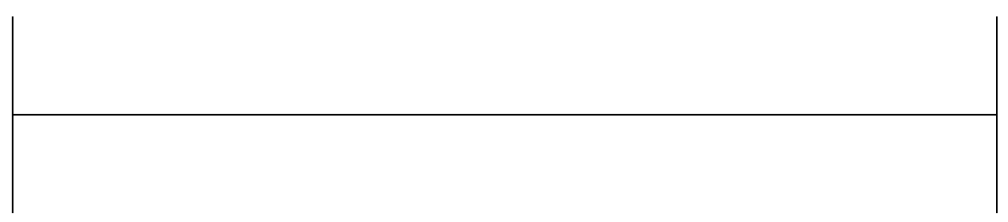

No excitement

Most possible excitement 
4. How much agitation do you feel?

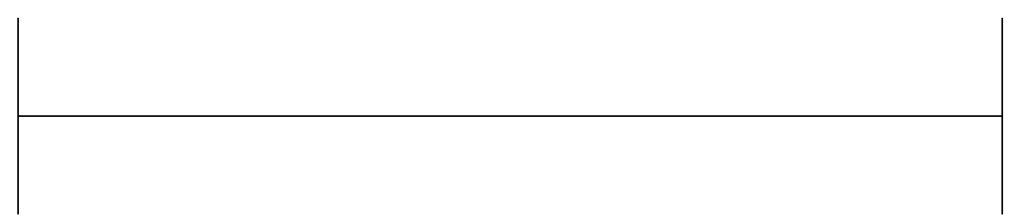

No agitation

Most possible agitation

5. Please indicate the EMOTION you feel the most AT THIS MOMENT: 


\section{Appendix E}

\section{AIMS SCALE}

Sex:

Date of

Birth: Date:

The following questions are about your experiences with music.

1. I will sometimes move my hand as if I were 'conducting' music

$\begin{array}{ccccc}1 & 2 & 3 & 4 & 5\end{array}$

2. When listening to music, I sometimes temporarily forget where I am

$\begin{array}{ccccc}1 & 2 & 3 & 4 & 5\end{array}$

3. I sometimes feel like I am 'one' with the music

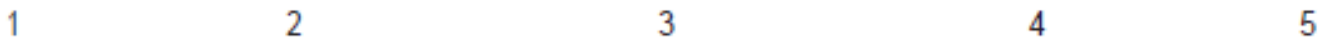

Strongly Disagree $\quad$ Somewhat Disagree Neutral (Neither Agree nor Disagree) Somewhat Agree Strongly Agree

4. When I listen to music I can get so caught up in it that I don't notice anything

$\begin{array}{ccccc}1 & 2 & 3 & 4 & 5 \\ \text { Strongly Disagree } & \text { Somewhat Disagree } & \text { Neutral (Neither Agree nor Disagree) } & \text { Somewhat Agree } & \text { Strongly Agree }\end{array}$

5. When I feel that nobody understands me, I often turn on some music

$\begin{array}{ccccc}1 & 2 & 3 & 4 & 5 \\ \text { Strongly Disagree } & \text { Somewhat Disagree } & \text { Neutral (Neither Agree nor Disagree) } & \text { Somewhat Agree } & \text { Strongly Agree }\end{array}$

6. I will stop everything that I'm doing in order to listen to a special song/piece of music that is playing

$1 \quad 2 \quad 3 \quad 405$

Strongly Disagree $\quad$ Somewhat Disagree Neutral (Neither Agree nor Disagree) Somewhat Agree Strongly Agree

7. I can imagine a song/piece of music so vividly that it holds my attention as if I were hearing it live

$\begin{array}{ccccc}1 & 2 & 3 & 4 & 5 \\ \text { Strongly Disagree } & \text { Somewhat Disagree } & \text { Neutral (Neither Agree nor Disagree) } & \text { Somewhat Agree } & \text { Strongly Agree }\end{array}$

8. When I hear good music I tend to lose my train of thought and forget what I was thinking about
1
2
3
4
5

Strongly Disagree Somewhat Disagree Neutral (Neither Agree nor Disagree) Somewhat Agree Strongly Agree 
9. Sometimes when listening to music I feel as if my mind can understand the whole world

$\begin{array}{ccccc}1 & 2 & 3 & 4 & 5 \\ \text { Strongly Disagree } & \text { Somewhat Disagree } & \text { Neutral (Neither Agree nor Disagree) } & \text { Somewhat Agree } & \text { Strongly Agree }\end{array}$

10. I sometimes feel that I understand the songwriter/composer's intentions completely

$\begin{array}{ccccc}1 & 2 & 3 & 4 & 5 \\ \text { Strongly Disagree } & \text { Somewhat Disagree } & \text { Neutral (Neither Agree nor Disagree) } & \text { Somewhat Agree } & \text { Strongly Agree }\end{array}$

11. I can change almost any sound into music by the way I listen to it

$\begin{array}{ccccc}1 & 2 & 3 & 4 & 5 \\ \text { Strongly Disagree } & \text { Somewhat Disagree } & \text { Neutral (Neither Agree nor Disagree) } & \text { Somewhat Agree } & \text { Strongly Agree }\end{array}$

12. I have stopped walking to listen to music that I came across on my path

$\begin{array}{ccccc}1 & 2 & 3 & 4 & 5 \\ \text { Strongly Disagree } & \text { Somewhat Disagree } & \text { Neutral (Neither Agree nor Disagree) } & \text { Somewhat Agree } & \text { Strongly Agree }\end{array}$

13. While listening to music, I may become so involved that I may forget about myself and my surroundings

$\begin{array}{ccccc}1 & 2 & 3 & 4 & 5 \\ \text { Strongly Disagree } & \text { Somewhat Disagree } & \text { Neutral (Neither Agree nor Disagree) } & \text { Somewhat Agree }\end{array}$

14. If I want to feel creative, I will turn on some music

$\begin{array}{ccccc}1 & 2 & 3 & 4 & 5 \\ \text { Strongly Disagree } & \text { Somewhat Disagree } & \text { Neutral (Neither Agree nor Disagree) } & \text { Somewhat Agree } & \text { Strongly Agree }\end{array}$

15. It is sometimes possible for me to be completely immersed in music and to feel as if my whole state of consciousness has been temporarily altered
$\begin{array}{lllll}1 & 2 & 3 & 4 & 5\end{array}$
Strongly Disagree Somewhat Disagree Neutral (Neither Agree nor Disagree) Somewhat Agree Strongly Agree

16. I know what people mean when they talk about mind-altering musical experiences
1
2
3
45

Strongly Disagree $\quad$ Somewhat Disagree $\quad$ Neutral (Neither Agree nor Disagree)

17. At times when listening to music, I feel more connected with other people
1
12
Strongly Disagree Somewhat Disagree Neutral (Neither Agree nor Disagree)
45
Somewhat Agree Strongly Agree

18. I find that different sound have different colors (e.g., red, blue)

$\begin{array}{ccccc}1 & 2 & 3 & 4 & 5 \\ \text { Strongly Disagree } & \text { Somewhat Disagree } & \text { Neutral (Neither Agree nor Disagree) } & \text { Somewhat Agree } & \text { Strongly Agree }\end{array}$


19. I spend as much time as I can every day listening to music

$\begin{array}{ccccc}1 & 2 & 3 & 4 & 5\end{array}$

20. Sometimes music makes me feel and experience things as I did when I was a child

$\begin{array}{lllll}1 & 2 & 3 & 4 & 5\end{array}$

Strongly Disagree Somewhat Disagree Neutral (Neither Agree nor Disagree) Somewhat Agree Strongly Agree

21. Sometimes I almost feel as if a song was written especially for/about me

$1 \quad 2 \quad 3 \quad 4 \quad 5$

Strongly Disagree Somewhat Disagree Neutral (Neither Agree nor Disagree) Somewhat Agree Strongly Agree

22. I sometimes make my movements/actions (opening doors, pushing buttons, stepping of curbs) coincide with the music

$1 \quad 2 \quad 3 \quad 4 \quad 5$

Strongly Disagree Somewhat Disagree Neutral (Neither Agree nor Disagree) Somewhat Agree Strongly Agree

23. I like to find patterns in everyday sounds

$\begin{array}{ccccc}1 & 2 & 3 & 4 & 5 \\ \text { Strongly Disagree } & \text { Somewhat Disagree } & \text { Neutral (Neither Agree nor Disagree) } & \text { Somewhat Agree } & \text { Strongly Agree }\end{array}$

24. If I want to feel creative, I will turn on some music

$\begin{array}{ccccc}1 & 2 & 3 & 4 & 5 \\ \text { Strongly Disagree } & \text { Somewhat Disagree } & \text { Neutral (Neither Agree nor Disagree) } & \text { Somewhat Agree } & \text { Strongly Agree }\end{array}$

25. Before I do an activity (e.g., exercise, study), I usually carefully consider what music to play along with it

$\begin{array}{ccccc}1 & 2 & 3 & 4 & 5\end{array}$

26. The sound of a speaking voice can be so fascinating to me that I can just go on listening to it $\begin{array}{lllll}1 & 2 & 3 & 4 & 5\end{array}$ Strongly Disagree Somewhat Disagree Neutral (Neither Agree nor Disagree) Somewhat Agree Strongly Agree

27. Music sometimes helps me 'step outside' my usual self and experience an entirely different state of being
1
2
3
Strongly Disagree Somewhat Disagree Neutral (Neither Agree nor Disagree)
4
Somewhat Agree
5

28. When listening to music, I often imagine the musicians playing the songs

$\begin{array}{ccccc}1 & 2 & 3 & 4 & 5\end{array}$


29. When listening to great music I sometimes feel as if I am being lifted into the air

$1 \quad 2 \quad 3 \quad 4 \quad 5$

Strongly Disagree Somewhat Disagree Neutral (Neither Agree nor Disagree) Somewhat Agree Strongly Agree

30. When I am listening to music, I can tune out everything else

$\begin{array}{lllll}1 & 2 & 3 & 4\end{array}$

Strongly Disagree $\quad$ Somewhat Disagree Neutral (Neither Agree nor Disagree) Somewhat Agree Strongly Agree

31. I sometimes see vivid images in my head when I listen to music

$\begin{array}{lllll}1 & 2 & 3 & 4 & 5\end{array}$

Strongly Disagree Somewhat Disagree $\quad$ Neutral (Neither Agree nor Disagree) Somewhat Agree Strongly Agree

32. I sometimes close my eyes so I can focus on the music I am listening to

$\begin{array}{lllll}1 & 2 & 3 & 4\end{array}$

Strongly Disagree Somewhat Disagree Neutral (Neither Agree nor Disagree) Somewhat Agree Strongly Agree

33. There are times when I will do nothing except listen to music

$\begin{array}{lllll}1 & 2 & 3 & 4 & 5\end{array}$

Strongly Disagree Somewhat Disagree Neutral (Neither Agree nor Disagree) Somewhat Agree Strongly Agree

34. I sometimes feel like I'm part of something bigger than myself when I listen to music

$\begin{array}{lllll}1 & 2 & 3 & 4 & 5\end{array}$

Strongly Disagree Somewhat Disagree Neutral (Neither Agree nor Disagree) Somewhat Agree Strongly Agree

www.smartlaboratory.org/aims 


\section{Appendix G}

Participant ID:

Date:

\section{$\underline{\text { MQ-I }}$}

1. Are you right- or left-handed?

Right Left

2. What is your first language?

3. Do you have normal hearing?

a) Do you have perfect pitch?

4. Do you listen to music?

b) How many hours each day?

c) What type or genre? (e.g., classical, rock, etc.)

d) What is your favorite type?

e) What are your reasons for listening to music?

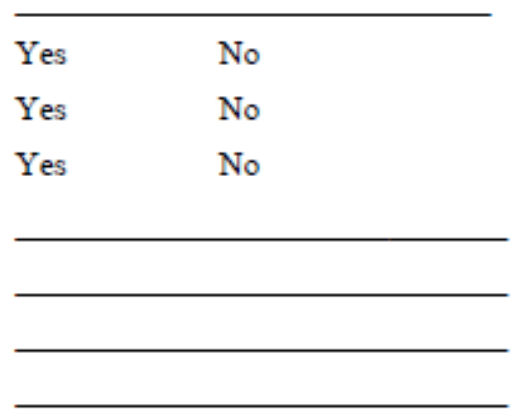

5. Is music important to you?

Yes No

f) How?

6. Do you consider yourself musical?

Yes

No

7. If you have ever played a musical instrument, please fill in the following table. Indicate the age range during which you played and how many years since you last played. Use a different line for each instrument.

\begin{tabular}{|c|c|c|c|c|}
\hline $\begin{array}{c}\text { Instrument } \\
\text { (e.g., piano, violin, voice) }\end{array}$ & $\begin{array}{c}\text { Type of Training } \\
\text { [e.g., self-taught, high school, } \\
\text { RCM (Royal Conservatory of Music)] }\end{array}$ & $\begin{array}{l}\text { Age Range } \\
\text { (e.g., \&-12) }\end{array}$ & $\begin{array}{l}\text { Years } \\
\text { Since }\end{array}$ & $\begin{array}{l}\text { Grade } \\
\text { (if RCM) }\end{array}$ \\
\hline & & & & \\
\hline & & & & \\
\hline & & & & \\
\hline & & & & \\
\hline
\end{tabular}

8. Are you currently musically active? Yes No

How? (e.g., lessons, jamming with friends, performing in an orchestra or band, etc.)

g) How many hours per week do you play in a group?

h) How many hours per week do you play by yourself? 
Participant $\mathbb{D}$ :

Date:

9. a) If you heard music during the study, please indicate how you FELT while listening:

- Very relaxed

- Somewhat relaxed

- Very agitated

- Somewhat agitated

- Very excited

- Somewhat excited

- Neutral

- I DID NOT HEAR MUSIC DURING THE STUDY

b) Please indicate the EMOTION you felt the MOST while listening (even if you didn't hear music):

c) Did you consider the music to be PLEASURABLE (Please circle one that best applies)?

Yes

No

d) If yes, please rate how pleasurable you considered it to be on a scale of 0 to 5 :

1

2

3

4

Not at all pleasurable Very

pleasurable

e) Were you familiar with any of the music?

Yes No

f) If yes, please rate how familiar you were with the music on a scale of 0 to 5 :

0

1

2

Not at all familiar
3

4 5 Very familiar 


\section{Appendix $\mathrm{H}$}

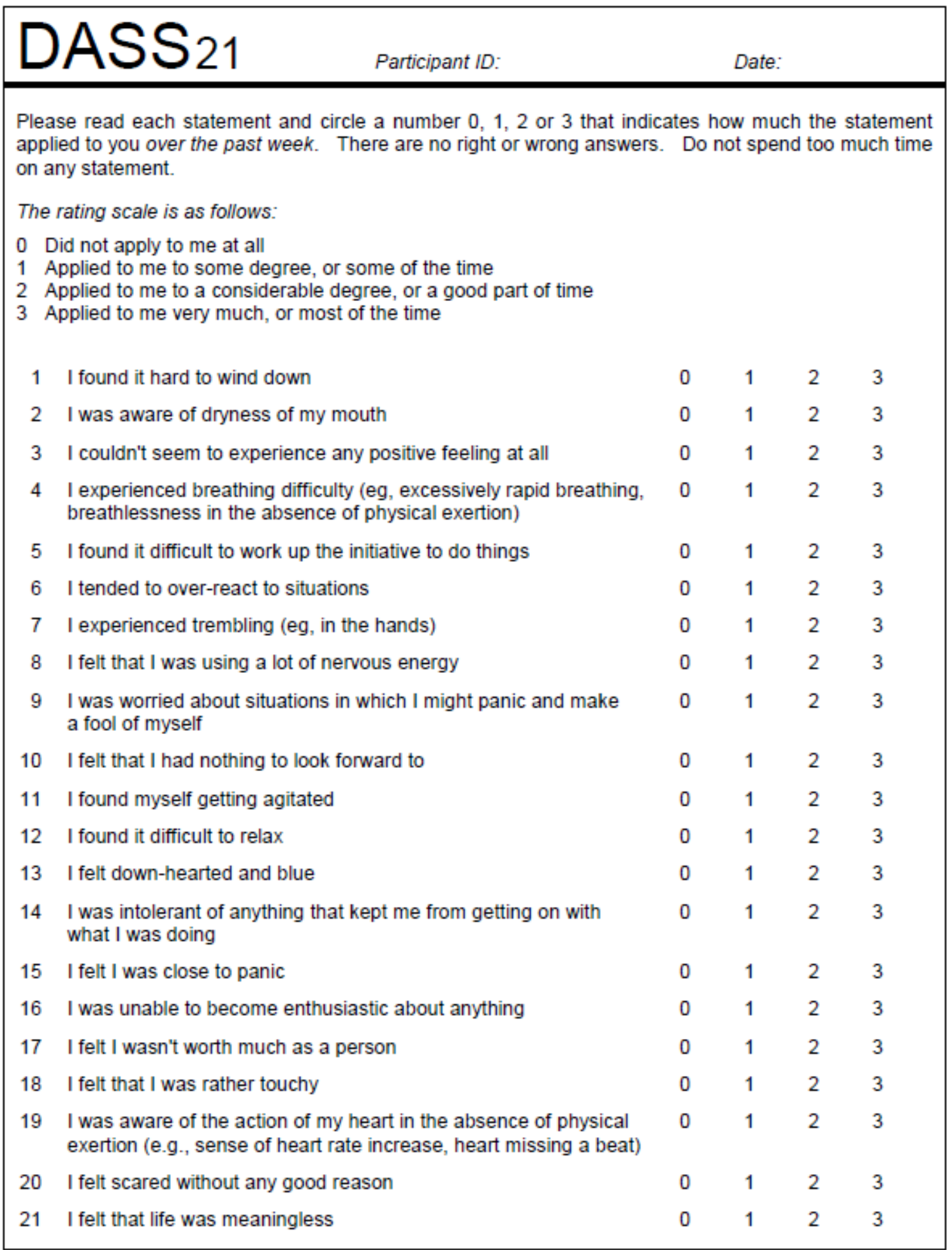




\section{Appendix I}

Participant ID:

Date:

\section{PSS}

The questions in this scale ask you about your feelings and thoughts during the last month. In each case, you will be asked to indicate by circling how often you felt or thought a certain way.

Age __ Gender (Circle): M F

$$
0=\text { Never } \quad 1=\text { Almost Never } \quad 2 \text { = Sometimes } \quad 3=\text { Fairly Often } \quad 4=\text { Very Often }
$$

1. In the last month, how often have you been upset because of something that happened unexpectedly?

2. In the last month, how often have you felt that you were unable to control the important things in your life?

$\begin{array}{lllll}0 & 1 & 2 & 3 & 4\end{array}$

3. In the last month, how often have you felt nervous and "stressed"? $\begin{array}{lllll}0 & 1 & 2 & 3 & 4\end{array}$

4. In the last month, how often have you felt confident about your ability to handle your personal problems?

5. In the last month, how often have you felt that things were going your way?

6. In the last month, how often have you found that you could not cope with all the things that you had to do?

7. In the last month, how often have you been able to control irritations in your life?

8. In the last month, how often have you felt that you were on top of things?.. $\quad \begin{array}{llllll}0 & 1 & 2 & 3 & 4\end{array}$

9. In the last month, how often have you been angered because of things that were outside of your control?

10. In the last month, how often have you felt difficulties were piling up so high that you could not overcome them? 
Appendix J

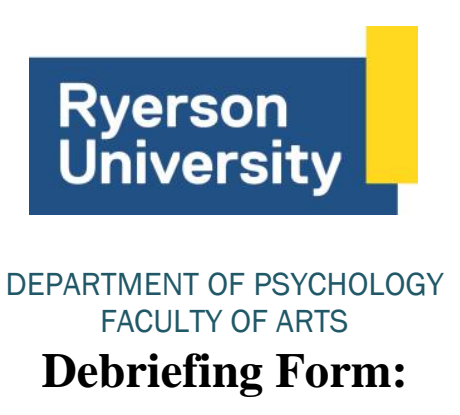

\section{Evaluating the Effect of Music on the Psychology of Language}

Exposure to chronic stress can be harmful to an individual's health at any age. According to Statistics Canada, more than 1 in 4 Canadians report daily stress levels in the high to severe range. Research has demonstrated a reliable association between perceived stress and disorders including depression, anxiety, chronic fatigue, cognitive impairment and age of mortality. Representing 15\% of the burden of disease in Canada, mental illness receives less than 6\% of health care dollars (Institute of Health Economics, 2008). Consequently, the development of low-cost strategies that minimize the effect of stress on the individual is paramount.

Music is a low-cost intervention that has been suggested to reduce stress in young adults following stress exposure (Chanda \& Levitin, 2013). Mixed findings have suggested that individuals can respond differently to music. More specifically, individuals that become more highly absorbed, or immersed into music while listening can recover more quickly after a stressful event, compared to individuals who do not. The potential influence of music absorption or the tendency to feel strong emotional responses to music, has not been explored and may explain previous negative findings. Finally, given the aging population, it is important to assess stress-reduction techniques in older adults, who may respond differently to psychosocial stressors.

The goal of the proposed project is to evaluate if music presented before a stressful experience can reduce stress, and to determine whether the degree of music absorption influences this effect. In this study, the independent, or predictor variable was the type of music presented (participant-selected, researcher-selected, pink noise control, or silence). The dependent, or outcome variables of interest include both your physiological (e.g., heart rate, blood pressure, sweat response) and subjective responses (questionnaire responses) recorded throughout the study. Some participants in the study listened to music or white noise, and some participants sat in silence. This was done in order to assess any differences in relation to the presence or absence of music on stress responses when presented before a stressful event. Any music presented that was chosen by the researcher has previously been validated as "calming". We did not initially make you aware of the true intent of the study, in order to avoid biasing your response on a subconscious level, which may influence your natural response to the stressful situation and the stimuli.

To assess the effects of a short-term psychological stressor on your physiology (e.g., sweat response) we used of the Trier Social Stress Test. Your heart rate, saliva, and blood pressure were taken throughout the experiment in order to investigate any changes in your physiology as a result of this stress test, along with subjective ratings of how "stressed" or uncomfortable the test made you feel. This test is widely used by researchers and has been shown to increase reported levels of subjective and physiological stress (e.g. increased heart rate) for a short duration. This task should not lead to long-lasting negative affect. In this test, you were asked to complete a timed "language task" in front of an expert panel while being video recorded. What we didn't tell you before is that the individuals who posed as evaluators were in fact research assistants from this lab. They did not actually 
analyze or evaluate your performance in any way. As well, your speech was not recorded, and footage from the video camera will not be used or analyzed. The task that you completed was unreasonably difficult and does not in any way reflect your personal aptitude or ability. This deception is extremely important in order to be able to examine the effect of music on the stress response. As you might imagine, knowing the true nature of the study might potentially (even unknowingly) affect how you respond to questionnaires and the language task.

In addition, you completed a battery of questionnaires that assessed the degree of music absorption, depression, anxiety, personality, emotional state, and general perceived stress. Your responses on these measures will allow us to examine whether any of these factors might alter the relationship between music and stress reactivity. It is important to note that although you answered some questions related to depression and anxiety, and it is a variable of interest in the study, these questionnaires are not used in any way to diagnose depression and/or anxiety. Participation in this study does not mean that you will be diagnosed with depression and/or anxiety.

As stated earlier, your data will be kept confidential. That is, your response to all questionnaires, your physiological (e.g., blood pressure) responses, and all demographic information collected will be absolutely confidential. Your name will be converted to a unique code number, (which is not associated with your name), and only people who are associated with this research will see your responses. All data collected is password protected and encrypted to ensure confidentiality of responses.

IMPORTANT: Please do not discuss this research with any other students or friends as they may be future participants and any pre-existing knowledge will render the data useless.

Your participation in this study is greatly appreciated. If you have any additional questions, or would like to have information regarding the results of the study, please feel free to contact the principal investigator, Katlyn Peck via email; katlyn.peck@psych.ryerson.ca, or phone; 416-979-5000 ext. 3233. This project has been approved by the Ryerson Research Ethics Board (REB \#2015-149). If you have any questions about your rights as a research participant, please contact the chair of Ryerson's REB Lynn Lavallée, Ph.D, at 416-979-5000 ext. 4791, or by email: rebchair@ryerson.ca

As an additional support, the on-call clinicians at Ryerson University's Centre for Student Development and Counselling (CSDC) are available by telephone (416-979-5195), email csdc@ ryerson.ca, or in-person counsel between 9am and 4:45pm, Monday-Friday in room JOR-07C in Jorgensen Hall.

\section{$\underline{\text { References }}$}

Chanda, M. L., \& Levitin, D. J. (2013). The neurochemistry of music. Trends in Cognitive Sciences, 17, 179-193. doi: $10.1016 /$ j.tics.2013.02.007

Institute of Health Economics (2008). How much should we spend on mental health? Alberta, Canada. Retrieved from http://www.ihe.ca/publications/how-much-should-we-spend-on-mental-health-.

\section{Future reading}

Pelletier, C. L. (2004). The effect of music on decreasing arousal due to stress: A meta-analysis. Journal of Music Therapy, 41, 192-214.

Thoma, M. V., Scholz, U., Ehlert, U., \& Nater, U. M. (2012). Listening to music and physiological and psychological functioning: The mediating role of emotion regulation and stress reactivity. Psychology \& Health, 27, 227-241. 


\section{References}

Acharya, U. R., Joseph, K. P., Kannathal, N., Lim, C. M., \& Suri, J. S. (2006). Heart rate variability: A review. Medical \& Biological Engineering \& Computing, 44, 1031-1051. doi: 10.1007/s11517-006-0119-0.

Adam, E. K., \& Kumari, M. (2009). Assessing salivary cortisol in large-scale, epidemiological research. Psychoneuroendocrinology, 34, 1423-1436. doi:10.1016/j.psyneuen.2009.06.011.

al'Absi, M., Petersen, K. L., \& Wittmers, L. E. (2002). Adrenocortical and hemodynamic predictors of pain perception in men and women. Pain, 96, 197-204. doi: 10.1016/S03043959(01)00447-X.

Allen, K. M., Blascovich, J., Tomaka, J., \& Kelsey, R. M. (1991). Presence of human friends and pet dogs as moderators of autonomic responses to stress in women. Journal of Personality and Social Psychology, 61, 582-589.

Allen, A. P., Kennedy, P. J., Cryan, J. F., Dinan, T. G., \& Clarke, G. (2014). Biological and psychological markers of stress in humans: Focus on the Trier Social Stress Test. Neuroscience and Biobehavioral Reviews, 38, 94-124. doi: https://doi.org/10.1016/jneurobiorev.2013.11.005.

Allen, A. P., Kennedy, P. J., Dockray, S., Cryan, J. F., Dinan, T. G., \& Clarke, G. (2017). The Trier Social Stress Test: Principles and practices. Neurobiology of Stress, 6, 113-126. doi:https://doi.org/10.1016/j.ynstr.2016.11.001.

Alvarsson, J. J., Wiens, S., \& Nilsson, M. E. (2010). Stress recovery during exposure to nature sound and environmental noise. International Journal of Environmental Research and Public Health, 7, 1036-1046. doi: 10.3390/ijerph7031036. 
Axelrod, J., \& Reisine, T. D. (1984). Stress hormones: Their interaction and regulation. Science, 224, 452-459. doi:10.1126/science.6143403.

Baum, A., \& Grunberg, N. (1995). Measurement of stress hormones. In S. Cohen, R. C., Kessler, \& L. U. Gordon (Eds.), Measuring stress: A guide for health and social scientists (pp. 175-192). New York: Oxford University Press.

Beddoe, A. E. \& Murphy, S. O. (2004). Does mindfulness decrease stress and foster empathy among nursing students? Journal of Nursing Education, 43, 305-312.

Berbel, P., Moix, J., \& Quintana, S., (2007). Music versus diazepam to reduce 895 preoperative anxiety: a randomized controlled clinical trial. Revista Espanola de Anestesiologia y Reanimacion, 54, 355-358.

Bernston, G. G., Bigger, J. T., Eckberg, D. L., Grossman, P., Kaufmann, P. G., Malik, M.,...Van Der Molen, M. W. (1997). Heart rate variability: Origins, methods, and interpretive caveats. Psychophysiology, 34, 623-648. doi: 10.1111/j.1469-8986.1997.tb02140.x.

Biondi, M., \& Picardi, A. (1999). Psychological stress and neuroendocrine function in humans: The last two decades of research. Psychotherapy and Psychosomatics, 68(3), 114-50.

Blandini, F., Martignoni, E., Sances, E., Bono, G., \& Nappi, G. (1995). Combined response of plasma and platelet catecholamines to different types of short-term stress. Life Sciences Including Pharmacology Letters, 56, 1113-1120. doi:10.1016/0024-3205(95)00048-B.

Blood, A. J., \& Zatorre, R. J. (2001). Intensely pleasurable responses to music correlate with activity in brain regions implicated in reward and emotion. PNAS, 98, 11818-11823. doi: 10.1073/pnas.191355898.

Boucsein, W., Fowles, D.C., Grimnes, S., Ben-Shakhar, G., Roth, W.T., Dawson, M.E., \& Filion, D.L (2012). Publication recommendations for electrodermal measurements. 
Psychophysiology, 49, 1017-1034. doi:10.1111/j.1469-8986.2012.01384.x.

Braithwaite, J. J., Watson, D. G., Jones, R., \& Rowe, M. (2013). A Guide for Analysing

Electrodermal Activity (EDA) \& Skin Conductance Responses (SCRs) for Psychological Experiments. (Revised version: 2.0). (Technical Report used for EDA manual).

Brindle, R. C., Ginty, A. T., \& Conklin, S. M. (2013). Is the association between depression and blunted cardiovascular stress reactions mediated by perceptions of stress? International Journal of Psychophysiology, 90, 66-72. doi: https://doi.org/10.1016/j.jpsycho.2013.06.003.

Buchanan, T. W., Tranel, D., \& Adolphs, R. (2006). Impaired memory retrieval correlates with individual differences in cortisol response but not autonomic response. Learning \& Memory, 13, 382-387. doi:10.1101/lm.206306/

Buske-Kirschbaum, A., Krieger, S., Wilkes, C., Rauh, W., Weiss, S., \& Hellhammer, D. H. (2007). Hypothalamic-pituitary-adrenal axis function and the cellular immune response in former preterm children. The Journal of Clinical Endocrinology \& Metabolism, 92, 34293435. doi: https://doi.org/10.1210/jc.2006-2223.

Cacioppo, J. T., Tassinary, \& L. G., \& Bernston, G. G. (2007). (Eds.). Handbook of psychophysiology, New York: Cambridge University Press.

Cannon, W. B. (1914). The emergency function of the adrenal medulla in pain and the major emotions. American Journal of Physiology, 33, 356-372.

Cannon, W. B. (1929). The sympathetic division of the autonomic system in relation to homeostasis. Archives of Neurology and Psychiatry, 22, 282-294. doi: 10.1001/archneurpsyc.1929.02220020098005.

Carney, R. M., Freedland, K. E., \& Veith, R. C. (2005). Depression, the autonomic nervous 
system, and coronary heart disease. Psychosomatic Medicine, 67, S29-S33. doi:

10.1097/01.psy.0000162254.61556.d5.

Carney, R. M., Freedland, K. E., Veith, R. C., Cryer, P. E., Skala, J. A., Lynch, T., \& Jaffe, A. S. (1999). Major depression, heart rate, and plasma norepinephrine in patients with coronary heart disease. Biological Psychiatry, 45, 458-43. doi: https://doi.org/10.1016/S00063233(98)00049-3.

Cecil, M. A., \& Forman, S. G. (1990). Effects of stress inoculation training and coworker support groups on teachers' stress. Journal of School Psychology, 28, 105-118. doi:https://doi.org/10.1016/0022-4405(90)90002-O.

Chahal, H. S., \& Drake, W. M. (2007). The endocrine system and ageing. Journal of Pathology, 211, 173-180. doi:10.1002/path.2110.

Chanda, M. L., \& Levitin, D. J. (2013). The neurochemistry of music. Trends in Cognitive Sciences, 17, 179-194. doi: org/10.1016/j.tics.2013.02.007.

Chandola, T., Britton, A., Brunner, E., Hemingway, H., Malik, M., Kumari, M.,...Marmot, M. (2008). Work stress and coronary heart disease: What are the mechanisms? European Heart Journal, 29, 640-648. doi: https://doi.org/10.1093/eurheartj/ehm584.

Childs, E., Dlugos, A., De Wit, H. (2010). Cardiovascular, hormonal, and emotional responses to the TSST in relation to sex and menstrual cycle phase. Psychophysiology, 47, 550-559. doi: 10.1111/j.1469-8986.2009.00961.x.

Chlan, L. L., Engeland, W. C., Anthony, A., \& Guttormson, J. (2007). Influence of music on the stress response in patients receiving mechanical ventilatory support: A pilot study. American Journal of Critical Care, 16, 141-145.

Chrousos, G. P. (2009). Stress and disorders of the stress system. Nature Reviews 
Endocrinology, 5, 374-381. doi: 10.1038/nrendo.2009.106.

Chrousos, G. P., \& Gold, P. (1992). The concept of stress system disorders: Overview of behavioral and physical homeostasis. The Journal of the American Medical Association, 267, 1244-1252. doi: 10.1001/jama.267.9.1244.

Clement, S., Tonini, A., Khatir, F., Schiaratura, L., \& Samson, S. (2012). Short and longer term effects of musical intervention in severe Alzheimer's disease. Music Perception, 29, 533541. doi: 10.1525/mp.2012.29.5.533.

Coffman, D. D., \& Adamek, M. S. (1999). The contributions of wind band participation to quality of life of senior adults. Music Therapy Perspectives, 17, 27-31. doi:https://doi.org/10.1093/mtp/17.1.27.

Cohen, S., Kamarck, T., \& Mermelstein, R. (1983). A global measure of perceived stress. Journal of Health and Social Behavior, 24, 385-396.

Conrad, C., Niess, H., Jauch, K.W., Bruns, C.J., Hartl, W., Welker, L., 2007. Overture for 924 growth hormone: requiem for interleukin-6? Crit. Care Med. 35, 2709-2713. doi: 10.1097/01.CCM.0000291648.99043.B9.

Creech, A., Hallam, S., McQueen, H., \& Varvarigou, M. (2013). The power of music in the lives of older adults. Research Studies in Music Education, 35, 87-102. $10.1177 / 1321103 X 13478862$.

Critchley, H. D. (2005). Neural mechanisms of autonomic, affective, and cognitive integration. The Journal of Comparative Neurology, 493, 154-166. doi: 10.1002/cne.20749.

Curtis, B. M., \& O'Keefe, J. H. (2002). Autonomic tone as a cardiovascular risk factor: The dangers of chronic fight or flight. Mayo Clinic Proceedings, 77, 45-54. doi: https://doi.org/10.4065/77.1.45. 
de Kloet, E. R. (1991). Brain corticosteroid receptor balance and homeostatic control. Frontiers in Neuroendocrinology, 12, 95-164.

Davies, H. W., Teschke, K., Kennedy, S. M., Hodgson, M. R., Hertzman, C., \& Demers, P. A. (2005). Occupational exposure to noise and mortality from acute myocardial infarction. Epidemiology, 16, 25-32. doi:10.1097/01.ede.0000147121.13399.bf.

Dawson, M. E., Schell, A. M., \& Filion, D. (2007). The electrodermal system. In: J. T. Cacioppo, L. G. Tassinary, \& G. G. Bernston, (Eds.). Handbook of psychophysiology, (3 ${ }^{\text {rd }}$ Ed.) New York: Cambridge University Press.

Dickerson, S. S., \& Kemeny, M. E. (2004). Acute stressors and cortisol responses: A theoretical integration and synthesis of laboratory research. Psychological Bulletin, 130, 355-391. doi: 10.1037/0033-2909.130.3.355.

Dishman, R. K., Nakamura, Y., Garcia, M. E., Thompson, R. W., Dunn, A. L., \& Blair, S. N. (2000). Heart rate variability, trait anxiety, and perceived stress among physically fit men and women. International Journal of Psychophysiology, 37, 121-133. doi:

Duncko, R., Cornwell, B., Cui, L., Merikangas, K.R., Grillon, C., (2007). Acute exposure to stress improves performance in trace eyeblink conditioning and spatial learning tasks in healthy men. Learning \& Memory, 14, 329-335. doi:10.1101/1m.483807.

Dunn, J. D., \& Orr, S. E. (1984). Differential plasma corticosterone responses to hippocampal stimulation. Experimental Brain Research, 54, 1-6. doi:10.1007/BF00235813.

Engert, V., Efanov, S. I., Duchesne, A., Vogel, S., Corbo, V., \& Pruessner, J. C. (2012). Differentiating anticipatory from reactive cortisol responses to psychosocial stress. Psychoneuroendocrinology, 38, 1328-1337. doi:http://dx.doi.org/10.1016/j.psyneuen.2012.11.018. 
Epel, E. S., Burke, H. M., \& Wolkowitz, O. M. (2007). The Psychoneuroendocrinology of Aging: Anabolic and Catabolic Hormones. In C. M. Aldwin, C. L. Park, \& A. Spiro III (Eds.), Handbook of health psychology and aging (pp. 119-141). New York: Guilford Press.

Escher, J., Hohmann, U., Anthenien, L., Dayer, E., Bosshard, C., Gaillard, R.C. (1993). Music during gastroscopy. Schweizerische Medizinische Wochenschrift, 123, 935 1354-1358.

Evans, G. W., Hygge, S., \& Bullinger, M. (1995). Chronic noise exposure and psychological stress. Psychological Science, 6, 333-338. doi: 10.1111/j.1467-9280.1995.tb.00522.x.

Faul, F., Erdfelder, E., Lang, A-G., \& Buchner, A. (2007). G*Power 3: A flexible statistical power analysis program for the social, behavioral, and biomedical sciences. Behavior Research Methods, 39, 175-191.

Fekedulegn, D. B., Andrew, M. E., Burchfiel, C. M., Violanti, J. M., Hartley, T. A., Charles, L. E., \& Miller, D. B. (2007). Area under the curve and other summary indicators of repeated waking cortisol measurements. Psychosomatic Medicine, 69, 651-659. doi: 10.1097/PSY.0b013e31814c405c.

Ferrari, E., Cravello, L., Muzzoni, B., Casarotti, D., Paltro, M., Solerte, S. B.,...Magri, F. (2001). Age-related changes of the hypothalamic-pituitary-adrenal axis: Pathophysiological correlates. European Journal of Endocrinology, 144, 319-329.

Fiocco, A. J., Wan, N., Weekes, N., Pim, H., \& Lupien, S. J. (2006). Diurnal cycle of salivary cortisol in older adult men and women with subjective complaints of memory deficits and/or depressive symptoms: Relation to cognitive functioning. The International Journal on the Biology of Stress, 9, 143-152. doi: http://dx.doi.org/10.1080/10253890600965674. 
Fohr, T., Tolvanen, A., Myllymaki, T., Jarvela-Reijonen, E., Rantala, S., Korpela, R.,...Kujala, U. M. (2015). Subjective stress, objective heart rate variability-based stress, and recovery on workdays among overweight and psychologically distressed individuals: A crosssectional study. Journal of Occupational Medicine and Toxicology, 10, 1-9. doi: 10.1186/s12995-015-0081-6.

Folkman, S., \& Lazarus, R. S. (1985). If it changes it must be a process: Study of emotion and coping during three stages of a college examination. Journal of Personality and Social Psychology, 48, 150-170. doi:10.1037/0022-3514.48.1.150.

Fukui, H., \& Yamashita, M. (2003). The effects of music and visual stress on testosterone and cortisol in men and women. Neuro Endocrinology Letters, 24, 173-180.

Fries, E., Hesse, J., Hellhammer, J., \& Hellhammer, D. H. (2005). A new view on hypocortisolism. Psychoneuroendocrinology, 30, 1010-1016. doi: https://doi.org/10.1016/j.psyneuen.2005.04.006.

Gaab, J., Rohleder, N., Nater, U. M., \& Ehlert, U. (2005). Psychological determinants of the cortisol stress response: The role of anticipatory cognitive appraisal. Psychoneuroendocrinology, 30, 599-610. doi: 10.1016/j.psyneuen.2005.02.001.

Garrido, S., \& Schubert, E. (2010). Individual differences in the enjoyment of negative emotion in music: A literature review and experiment. Music Perception, 28, 279-296. doi: 10.1525/MP.2011.28.3.279.

Gembris, H. (2008, September). Musical activities in the third age: An empirical study with amateur musicians. In Second European Conference on Developmental Psychology of Music, Roehampton University, England (pp. 10-12).

Gerdner, L. A. (2000). Effects of individualized versus classical "relaxation" music on the 
frequency of agitation in elderly persons with Alzheimer's disease and related disorders. International Psychogeriatrics, 12, 49-65. doi: 10.1017/S10416102000006190.

Glaser, R., \& Kiecolt-Glaser, J. K. (2005). Stress-induced immune dysfunction: Implications for health. Nature Reviews Immunology, 5, 243-251. doi: 10.1038/nri1571.

Gloster, A. T., Rhoades, H. M., Novy, D., Klotsche, J., Senior, A., Kunik, M.,...Stanley, M. A. (2008). Psychometric properties of the depression anxiety and stress scale-21 in older adult primary care patients. Journal of Affective Disorders, 110, 248-259. doi: 10.1016/j.jad.2008.01.023.

Gold, P. W., \& Chrousos, G. P. (2002). Organization of the stress system and its dysregulation in melancholic and atypical depression: high vs low CRH/NE states. Molecular Psychiatry, 7, 254-275. doi: 110.1038/sj.mp.4001032.

Gold, P. W., Goodwin, F. K., \& Chrousos, G. P. (1988). Clinical and biochemical manifestations of depression: Relation to the neurobiology of stress. New England Journal of Medicine, 319, 413-420. doi: 10.1056/NEJM198808113190606.

Gold, S. M., Zakowski, S. G., Valdimarsdottir, H. B., \& Bovbjerg, D. H. (2004). Higher Beck depression scores predict delayed epinephrine recovery after acute psychological stress independent of baseline levels of stress and mood, Biological Psychology, 67, 261-273.

Goldstein, D. S. (2001). The autonomic nervous system in health and disease. New York, NY: Marcel Dekker, Inc.

Goldstein, D. S., \& Kopin, I. J. (2007). Evolution of concepts of stress. Stress, 10, 109-120. doi: $10.1018 / 10253890701288935$.

Goldstein, D. S., \& McEwen, B. S. (2002). Allostasis, homeostasis, and the nature of stress. The International Journal on the Biology of Stress, 5, 55-58. 
http://dx.doi.org/10.1080/102538902900012345.

Granger, D. A., Hibel, L. C., Fortunato, C. K., \& Kapelewski, C. H. (2009). Medication effects on salivary cortisol: Tactics and strategy to minimize impact in behavioral and developmental science. Psychoneuroendocrinology, 34, 1437-1448. doi: 10.1016/j.psyneuen.2009.06.017.

Grippo, A. J., \& Johnson, A. K. (2002). Biological mechanisms in the relationship between depression and heart disease. Neuroscience \& Biobehavioral Reviews, 26, 941-962. doi: https://doi.org/10.1016/S0149-7634(03)00003-4.

Grippo, A. J. Beltz, T. G., Weiss, R. M., \& Johnson, A. K. (2006). The effects of chronic fluoxetine treatment on chronic mild stress-induced cardiovascular changes and anhedonia. Biological Psychiatry, 59, 309-316. doi: https://doi.org/10.1016/j.biopsych.2005.07.010.

Grossman, P., Niemann, L., Schmidt, S., \& Walach, H. Mindfulness and its role in psychological well-being. Journal of Personality and Social Psychology, 84, 822-848. doi:10.1016/S0022-399(03)00573-7.

Guimont, C., Brisson, C., Dagenais, G. R., Milot, A., Vezina, M., Masse, B.,...Blanchette, C. (2006). Effects of job strain on blood pressure: A prospective study of male and female white-collar workers. American Journal of Public Health, 96, 1436-1443. doi:

\subsection{5/AJPH.2004.057679}

Gunnar, M. R., \& Donzella, B. (2002). Social regulation of the cortisol levels in early human development. Psychoneuroendocrinology, 27, 199-220. doi:https://doi.org/10.1016/S0306-4530(01)00045-2.

Gunnar, M. R., \& Quevedo, K. (2007). The neurobiology of stress and development. Annual 
Review of Psychology, 58, 145-173. doi:

https://doi.org/10.1146/annurev.psych.58.110405.085605.

Gunnar, M. R., \& White, B. P. (2001). Salivary cortisol measures in infant and child assessment. In Singer, L. T., Zeskind, P. S. (Eds.). Biobehavioral assessment of the infant. Guilford Press: New York.

Gunnar, M. R., Talge, N. M., \& Herrera, A. (2009). Stressor paradigms in developmental studies: What does and does not work to produce mean increases in salivary cortisol. Psychoneuroendocrinology, 34, 953-967. doi: 10.1016/j.psyneuen.2009.02.010.

Hall, M., Vasko, R., Buysse, D., Ombao, H., Chen, Q., Cashmere, J. D.,...Thayer, J. F. (2004). Acute stress affects heart rate variability during sleep. Psychosomatic Medicine, 66, 5662. doi: : 10.1097/01.PSY.0000106884.58744.09.

Hawkley, L. C., \& Cacioppo, J. T. (2004). Stress and the aging immune system. Brain, Behavior, and Immunity, 18, 114-119. doi:10.1016/j.bbi.2003.09.005.

Hays, T., \& Minichiello, V. (2005). The meaning of music in the lives of older people: A qualitative study. Psychology of Music, 33, 437-451. doi: 10.1177/0305735605056160.

Heim, C., Ehlert, U., \& Hellhammer, D. H. (2000). The potential role of hypocortisolism in the pathophysiology of stress-related bodily disorders. Psychoneuroendocrinology, 25, 1-35. doi: https://doi.org.10.1016/S0306-4530(99)00035-9.

Hellhammer, D. H., Wust, S., Kudielka, B. M. (2008). Salivary cortisol as a biomarker in stress research. Psychoneuroendocrinology, 34, 163-171. doi: 10.1016/j.psyneuen.2008.10.026.

Henry, J. P. (1992). Biological basis of the stress response. Integrative Physiological and Behavioral Science, 27, 66-83. doi:10.1007/BF02691093.

Henry, J. P., \& Crawford, J. R. (2005). The short-form version of the Depression Anxiety Stress 
Scales (DASS-21): Construct validity and normative data in a large non-clinical sample. British Journal of Clinical Psychology, 44, 227-239. doi: 10.1348/014466505X29657.

Herman, J. P., \& Cullinan, W. E. (1997). Neurocircuitry of stress: Central control of the hypothalamo-pituitary-adrenocortical axis. Trends in Neurosciences, 20, 78-84. doi: https://doi.org/10.1016/S0166-2236(96)10069-2.

Herman, J. P., Ostrander, M. M., Mueller, N. K., \& Figueredo, H. (2005). Limbic system mechanisms of stress regulation: Hypothalamo-pituitary-adrenocortical axis. Progress in Neuro-Psychopharmacology and Biological Psychiatry, 29, 1201-1213. doi: https://doi.org/10.1016/j.pnpbp.2005.08.006.

Het, S., Rohleder, N., Schoofs, D., Kirschbaum, C., \& Wolf, O. T. (2009). Neuroendocrine and psychometric evaluation of a placebo version of the "Trier Social Stress Test". Psychoneuroendocrinology, 34, 1075-1086. doi: 10.1016/j.psyneuen.2009.02.008.

Hills, P., \& Argyle, M. (1998). Musical and religious experiences and their relationship to happiness. Personality and Individual Differences, 25, 91-102. doi:10.1016/S01918869(98)00004-X.

Hines, E. A., Jr., \& Brown, G. E. (1932). Standard stimulus for measuring vasomotor reactions. Its application in the study of hypertension. Proceedings of the Staff Meeting of the Mayo Clinic, 1932, 7, 332-335.

Hjortskov, N., Rissen, D., Blangsted, A. K., Fallentin, N., Lundberg, U., \& Sogaard, K. (2004). The effect of mental stress on heart rate variability and blood pressure during computer work. European Journal of Applied Physiology, 92, 84-89. doi: 10.1007/s00421-0041055-z.

Hoaglin, D. C., \& Iglewicz, B. (1987). Fine-tuning some resistant rules for outlier labeling. 
Journal of the American Statistical Association, 82, 1147-1149. doi: 10.2307/2289392.

Hoge, E. A., Bui, E., Marques, L., Metcalf, C. A., Morris, L. K., Robinaugh, D. J.,...Simon, N. M. (2013). Randomized controlled trial of mindfulness meditation for generalized anxiety disorder: Effects on anxiety and stress reactivity. The Journal of Clinical Psychiatry, 74, 786-792. doi:http://dx.doi.org/10.4088/JCP.12m08083.

Hogue, J. D., Crimmins, A. M., \& Kahn, J. H. (2016). “So sad and slow, so why can’t I turn off the radio": The effects of gender, depression, and absorption on liking music that induces sadness and music that induces happiness. Psychology of Music, 44, 816-829. doi:10.1177/0305735615594489.

Huron, D. (1992). The ramp archetype and the maintenance of passive auditory attention. Music Perception, 10, 83-92. doi:10.2307/40285540.

Ising, H., \& Braun, C. (2000). Acute and chronic endocrine effects of noise: Review of the research conducted at the Institute for water, soil, and air hygiene. Noise \& Health, 2, 724.

Janata, P., Tomic, S. T., \& Rakowski, S. K. (2007). Characterization of music-evoked autobiographical memories. Memory, 15, 845-860. doi: 10.1080/09658210701734593.

Jansen, A. S. P., Nguyen, X. V., Karpitskiy, V., Mettenleiter, T. C., \& Loewy, A. D. (1995). Central command neurons of the sympathetic nervous system: Basis of the fight or flight response. Science, 270, 644-646.

Jezova, D., Makatsori, A., Duncko, R., Moncek, F., \& Jakubek, M. (2004). High trait anxiety in health subjects is associated with low neuroendocrine activity during psychosocial stress. Progress in Neuropsychopharmacology \& Biological Psychiatry, 28, 1331-1336. doi:10.1016/j.pnpbp.2004.08.005. 
Jiang, J., Zhou, L., Rickson, D., \& Jiang, C. (2013). The effects of sedative and stimulative music on stress reduction depend on music preference. The Arts in Psychotherapy, 40, 201-205. doi:http://dx.doi.org/10.1016/j.aip.2013.02.002.

Johnson, E. O., Kamilaris, T. C., Chrousos, G. P., \& Gold, P. W. (1992). Mechanisms of stress: A dynamic overview of hormonal and behavioral homeostasis. Neuroscience and Biobehavioral Reviews, 16, 115-130.

Juslin, P. N., \& Laukka, P. (2004). Expression, perception, and induction of musical emotions: A review and a questionnaire study of everyday listening. Journal of New Music Research, 33, 217-238. doi: 10.1080/0929821042000317813.

Juslin, P. N., \& Vastfjall, D. (2008). Emotional responses to music: The need to consider underlying mechanisms. Behavioral and Brain Sciences, 31, 559-621. doi: 10.1017/S0140525X08005293.

Juster, R. P., McEwen, B. S., \& Lupien, S. J. (2010). Allostatic load biomarkers of chronic stress and impact on health and cognition. Neuroscience \& Biobehavioral Reviews, 35, 2-16. https://doi.org/10.1016/j.neubiorev. 2009.10.002.

Juster, R. P., Bizik, G., Picard, M., Arsenault-Lapierre, G., Sindi, S., Trepanier, L., ...Lupien, S. J. (2011). A transdisciplinary perspective of chronic stress in relation to psychopathology throughout life span development. Development and Psychopathology, 23, 725-776. doi: 10.1017/S0954794110000289.

Juster, R. P., Raymond, C., Bisson Desrochers, A., Bourdon, O., Durand, N., Wan, N.,...Lupien, S. J. (2016). Sex hormones adjust "sex-specific" reactive and diurnal cortisol profiles. Psychoneuroendocrinology, 63, 282-290. doi: http://dx.doi.org/10.1016/j.psyneuen.2015.10.012. 
Kabat-Zinn, J. (1982). An outpatient program in behavioral medicine for chronic pain patients based on the practice of mindfulness meditation: Theoretical considerations and preliminary results. General Hospital Psychiatry, 4, 33-47.

Kabat-Zinn, J. (1990). Full catastrophe living: Using the wisdom of your body and mind to face stress, pain and illness. New York, NY: Random House LLC

Kaltas, G. A., \& Chrousos, G. P. (2007). The neuroendocrinology of stress. In J. T. Cacioppo, L. G. Tassinary, \& G. G. Bernston (Eds.). Handbook of psychophysiology (pp. 303-318). New York: Cambridge University Press.

Kaplan, S. (1995). The restorative benefits of nature: Toward an integrative framework. Journal of Environmental Psychology, 15, 169-182. doi: https://doi.org/10.1016/02724944(95)90001-2.

Kelley, A. E. (2004). Ventral striatal control of appetitive motivation: Role in ingestive behavior and reward-related learning. Neuroscience and Biobehavioral Reviews, 27, 765-776. doi:10.1016/j.neubiorev.2003.11.015.

Kemeny, M. E. (2003). The psychobiology of stress. Current Directions in Psychological Science, 12, 124-129. doi: 10.1111/1467-8721.01246.

Khalfa, S., Peretz, I., Blondin, J-P., \& Manon, R. (2002). Event-related skin conductance responses to musical emotions in humans. Neuroscience Letters, 328, 145-149. doi: https://doi.org/10.1016/S0304-3940(02)00462-7.

Khalfa, S., Dalla Bella, S., Roy, M., Peretz, I., \& Lupien, S. J. (2003). Effects of relaxing music on salivary cortisol level after psychological stress. Annuals of the New York Academy of Science, 999, 374-376. doi: 10.1196/annuals.1284.045.

King, S. L., \& Hegadoren, K. M. (2002). Stress hormones: How do they measure up? Biological 
Research for Nursing, 4, 315-318.

Kirschbaum, C., \& Hellhammer, D. H. (1989). Salivary cortisol in psychobiological research: An overview. Neuropsychobiology, 22, 150-160. doi: 10.1159/000118611.

Kirschbaum, C., Pirke, K. M., \& Hellhammer, D. H. (1993). “The Trier Social Stress Test” - A tool for investigating psychobiological stress responses in a laboratory setting. Neuropsychobiology, 28, 76-81. doi:https://doi.org/10.1159/000119004.

Kirschbaum, C., Wust, S., \& Hellhammer, D. H. (1992). Consistent sex differences in cortisol responses to psychological stress. Psychosomatic Medicine, 54, 648-657.

Kirschbaum, C., Kudielka, B. M., Gaab, J., Schommer, N. C., \& Hellhammer, D. H. (1999). Impact of gender, menstrual cycle phase, and oral contraceptives on the activity of the hypothalamus-pituitary-adrenal axis. Psychsomatic Medicine, 61, 154-162.

Kiselica, M. S., Baker, S. B., Thomas, R. N., \& Reedy, S. (1994). Effects of stress inoculation training on anxiety, stress, and academic performance among adolescents. Journal of Counseling Psychology, 41(3), 335-342. http://dx.doi.org/10.1037/0022-0167.41.3.335

Kleiger, R. E., Stein, P. K., \& Bigger, T. Jr. (2005). Heart rate variability: Measurement and clinical utility. Annals of Noninvasive Electrocardiology, 10, 88-101. doi:10.1111/j.1542474X.2005.10101.x.

Kleiger, R. E., Miller, J. P., Bigger, T. Jr.., \& Moss, A. J. (1987). Decreased heart rate variability and its association with increased mortality after acute myocardial infarction. The American Journal of Cardiology, 59, 256-262. doi: https://doi.org/10.1016/00029149(87)90795-8.

Klein, L. C., \& Corwin, E. J. (2007). Homeostasis and the stress response. In E. J. Corwin (Ed.), Handbook of Pathophysiology (3rd ed.) (pp. 159-172). Philadelphia, PA: Lippincott 
Williams \& Wilkins.

Knight, W. E. J., \& Rickard, N. S. (2001). Relaxing music prevents stress-induced increases in subjective anxiety, systolic blood pressure, and heart rate in healthy males and females. Journal of Music Therapy, 38, 254-272. doi: 10.1093/jmt/38.4.254.

Koelsch, S. \& Jancke, L. (2015). Music and the heart. European Heart Journal, 36, 3043-3049. doi: https://doi.org/10.1093/eurheartj/ehv430.

Koelsch, S., Fritz, T., Müller, K., \& Friederici, A. D. (2006). Investigating emotion with music: an fMRI study. Human brain mapping, 27(3), 239-250. doi: 10.1002/hbm.20180.

Krantz, D. S., \& McCeney, M. K. (2002). Effects of psychological and social factors on organic disease: A critical assessment of research on coronary heart disease. Annual Reviews of Psychology, 53, 341-369. doi:https://doi org.ezproxy.lib.ryerson.ca/10.1146/annurev.psych.53.100901.135.208.

Kreutz, G., Ott, D., Teichmann, D., Osawa, P., \& Vaitl, D. (2008). Using music to induce emotions: Influences of musical preferences and absorption. Psychology of Music, 36, 101-126. doi: 10.1177/0305735607082623.

Kudielka, B. M., \& Kirschbaum, C. (2003). Awakening cortisol responses are influenced by health status and awakening time but not by menstrual cycle phase. Psychoneuroendocrinology, 28, 35-47.

Kudielka, B. M., \& Kirschbaum, C. (2005). Sex differences in HPA axis responses to stress: A review. Biological Psychology, 69, 113-132. doi: 10.1016/j.biopsycho.2004.11.009.

Kudielka, B. M., \& Wust, S. (2010). Human models in acute and chronic stress: Assessing determinants of individual hypothalamus-pituitary-adrenal axis activity and reactivity. The International Journal on the Biology of Stress, 13, 1-14. doi: 
http://dx.doi.org/10.3109/10253890902874913.

Kudielka, B. M., Buske-Kirschbaum, A., Hellhammer, D. H., \& Kirschbaum, C. (2004). HPA axis responses to laboratory psychosocial stress in healthy elderly adults, younger adults, and children: Impact of age and gender. Psychoneuroendocrinology, 29, 83-98. doi: 10.1016/S0306-4530(02)00146-4.

Kudielka, B. M., Schommer, N. C., Hellhammer, D. H., \& Kirschbaum, C. (2004). Acute HPA axis responses, heart rate, and mood changes to psychosocial stress (TSST) in humans at different times of day. Psychoneuroendocrinology, 29, 983-992. doi: 10.1016/j.psyneuen.2003.09.009.

Kudielka, B. M., Gierens, A., Hellhammer, D. H., Wust, S., \& Schlotz, W. (2012). Salivary cortisol in ambulatory assessment - Some dos, some don'ts, and some open questions. Psychosomatic Medicine, 74, 418-431. doi: https://dx.doi.org/10.1097/PSY.Ob013e31825434c7.

Kudielka, B. M., Schmidt-Reinwald, A. K., Hellhammer, D. H., Schurmeyer, T., \& Kirschbaum, C. (2000). Psychosocial stress and HPA functioning: No evidence for a reduced resilience in healthy elderly men. Stress: The International Journal on the Biology of Stress, 3, 229240. doi: $10.3109 / 10253890009001127$.

Kudielka, B. M., Hellhammer, J., Hellhammer, D. H., Wolf, O. T., Pirke, K-M., Varadi, E.,...Kirschbaum, C. (1998). Sex differences in endocrine and psychological responses to psychosocial stress in healthy elderly subjects and the impact of a 2-week dehydroepiandrosterone treatment. Journal of Clinical Endocrinology \& Metabolism, 83, 1756-1761. doi: https://doi.org/10.1210/jcem.83.5.4758.

Kumsta, R., Entringer, S., Hellhammer, D. H., \& Wust, S. (2007). Cortisol and ACTH responses 
to psychosocial stress are modulated by corticosteroid binding globulin levels. Psychoneuroendocrinology, 32, 1153-1157. doi:10.1016/j.psyneuen.2007.08.007.

Labbé, E., Schmidt, N., Babin, J., \& Pharr, M. (2007). Coping with stress: The effectiveness of different types of music. Applied Psychophysiological Biofeedback, 32, 163-168. doi: 10.1007/s10484-007-9043-9.

Lake, C. R., Pickar, D., Ziegler, M. G., Lipper, S., Slater, S., \& Murphy, D. L. (1982). High plasma NE levels in patients with major affective disorder. American Journal of Psychiatry, 139, 1315-1318.

Landfield, P. W., Baskin, R. K., \& Pitler, T. A. (1981). Brain aging correlates: Retardation by hormonal-pharmacological treatments. Science, 214, 581-584. doi: 10.1126/science.6270791.

Laukka, P. (2007). Uses of music and psychological well-being among the elderly. Journal of Happiness Studies, 8, 215-241. doi: 10.1007/s10902-006-9024-3.

Lazarus, R. S. (1966). Psychological stress and the coping process. New York: McGraw-Hill.

Lee, J., Tsunetsugu, Y., Takayama, N., Park, B-J., Li, Q., Song, C.,...Miyazaki, Y. (2014). Influence of forest therapy on cardiovascular relaxation in young adults. Evidence-Based Complementary Medicine, 1-7. doi:http://dx.doi.org/10.1155/2014/834360.

Lefèvre, M., Carlier, M., Champelovier, P., Lambert, J., Laumon, B., \& Evrard, A. (2017). Effects of aircraft noise exposure on saliva cortisol near airports in France. Occupational and Environmental Medicine, 74(8), 612. doi:http://dx.doi.org.ezproxy.lib.ryerson.ca/10.1136/oemed-2016-104208

Levy, B. R., Hausdorff, J. M., Hencke, R., \& Wei, J. Y. (2000). Reducing cardiovascular stress with positive self-stereotypes of aging. The Journals of Gerontology Series B: 
Psychological Sciences and Social Sciences, 55, 205-213. doi:

10.1093/geronb/55.4.P205.

Li, Q., Morimoto, K., Kobayashi, M., Inagaki, H., Katsumata, M., Hirata, Y.,...Miyazaki, Y. (2008). A forest bathing trip increases human natural killer activity and expression of anti-cancer proteins in female subjects. Journal of Biological Regulators \& Homeostatic Agents, 22, 45-55.

Limb, C. J. (2006). Structural and functional neural correlates of music perception. The Anatomical Record, 288A, 435-446. doi:10.1002/ar.a.201316.

Linneman, A., Ditzen, B., Strahler, J., Doerr, J. M., \& Nater, U. M. (2015). Music listening as a means of stress reduction in daily life. Psychoneuroendocrinology, 60, 82-90. doi: https://doi.org/10.1016/j.psyneuen.2015.06.008.

Liu, J. C. J., Verhulst, S., Massar, S. A. A., \& Chee, M. W. L. (2015). Sleep deprived and sweating it out: The effects of total sleep deprivation on skin conductance reactivity to psychosocial stress. Sleep, 38, 155-159. doi:https://doi.org/10.5665/sleep.4336.

Liu, J. J. W., Ein, N., Peck, K., Huang, V., Pruessner, J. C., \& Vickers, K. (2017). Sex differences in salivary cortisol reactivity to the Trier Social Stress Test (TSST): A metaanalysis. Psychoneuroendocrinology, 82, 26-37. doi:http://dx.doi.org/10.1016/j.psyneuen.2017.04.007.

Lovallo, W. R., \& Thomas, T. L. (2000). Stress hormones in psychophysiological research. In: J. T. Cacioppo, L. G. Tassinary, \& G. G. Bernston, (Eds.). Handbook of psychophysiology, New York: Cambridge University Press.

Loxton, N. J., Mitchell, R., Dingle, G. A., \& Sharman, L. S. (2016). How to tame your BAS: Reward sensitivity and music involvement. Personality and Individual Differences, 97, 
35-39. doi: http://dx.doi.org/10.1016/j.paid2016.03.018.

Lupien, S. J., McEwen, B. S., Gunnar, M. R., \& Heim, C. (2009). Effects of stress throughout the lifespan on the brain, behaviour and cognition. Nature Reviews Neuroscience, 10, 434-445. doi: 10.1038/nrn2639.

Lupien, S. J., Ouellet-Morin, I., Trépanier, L., Juster, R. P., Marin, M. F., Francois, N.,...,Corbo, K. (2013). The destress for success program: Effects of a stress education program on cortisol levels and depressive symptomatology in adolescents making the transition to high school. Neuroscience, 249, 74-87. https://doi.org/10.1016/j.neuroscience.2013.01.057.

MacDonald, R., Mitchell, L., Dillon, T., Serpell, M., Davies, J., \& Ashley, E. (2003). An empirical investigation of the anxiolytic and pain reducing effects of music. Psychology of Music, 31, 187-203. doi: 10.1177/0305735603031002294.

Markovitz, J. H., Matthews, K. A., Whooley, M., Lewis, C. E., \& Greenlund, K. J. (2004). Increases in job strain are associated with incident hypertension in the CARDIA study. Annals of Behavioral Medicine, 28, 4-9. doi: https://doi.org/10.1207/s15324796abm2801_2.

Mason, J. W. (1968). A review of psychoendocrine research on the pituitary-adrenal cortical system. Psychosomatic Medicine, 30.

Mather, M., \& Carstensen, L. L (2005). Aging and motivated cognition: The positivity effect in attention and memory. TRENDS in Cognitive Sciences, 9, 496-502. doi:10.1016/j.tics.2005.08.005.

McEwen, B. S. (2005). Stressed or stressed out: What is the difference? Journal of Psychiatry and Neuroscience, 30, 315-318. 
McEwen, B. S. (2007). Physiology and neurobiology of stress and adaptation: A central role of the brain. Physiological Reviews, 87, 873-904. doi: 10.1152/physrev.00041.2006.

McEwen, B. S. (2008). Central effects of stress hormones in health and disease:

Understanding the protective and damaging effects of stress and stress mediators. European Journal of Pharmacology, 538, 174-185. doi: 10.1016/j.ejphar.2007.11.071.

McEwen, B. S., \& Stellar, E. (1993). Stress and the individual: Mechanisms leading to disease. Archives of International Medicine, 153, 2093-2101. doi:

10.1001/archinte.1993.00410180039004.

McKee, S. A., Sinha, R., Weinberger, A. H., Sofuoglu, M., Harrison, E. L. R.,...Wanzer, J. (2011). Stress decreases the ability to resist smoking and potentiates smoking intensity and reward. Journal of Psychopharmacology, 25, 490-502. doi:

$10.1177 / 0269881110376694$.

McRae, A. L., Saladin, M. E., Brady, K. T., Upadhyaya, H., Back, S. E., \& Timmerman, M. (2006). Stress reactivity: Biological and subjective responses to the cold pressor and Trier Social stressors. Human Psychopharmacology Clinical and Experimental,21, 377-385. doi:10.1002/hup.778.

Meaney, M. J. (2001). Maternal care, gene expression, and the transmission of individual differences in stress reactivity across generations. Annual Review of Neuroscience, 24, 161-192.

Meaney, M., Aitken, D., Bhatnager, S., Vanberkel, C., \& Sapolsky, R. (1988). Effects of neonatal handling on age-related impairments associated with the hippocampus. Science, 239, 766-769. doi: 10.1126/science.3340858.

Meichenbaum, D. H. (1972). Cognitive modification of test anxious college students. Journal of 
Consulting and Clinical Psychology, 39, 370-380.

Meichenbaum, D. H., \& Turk, D. (1976). The cognitive-behavioural management of anxiety, anger and pain. In P. O. Davidson (Ed.), The behavioural management of anxiety, depression, and pain (pp. 1-34). New York: Brunner/Mazel.

Menon, V., \& Levitin, D. J. (2006). The rewards of music listening: Response and physiological connectivity of the mesolimbic system. NeuroImage, $28,175-184$. doi: 10.1016/j.neuroimage.2005.05.053.

Miller, G. E., Cohen, S., \& Ritchey, A. K. (2002). Chronic psychological stress and the regulation of pro-inflammatory cytokines: A glucocorticoid-resistance model. Health Psychology, 21(6), 531-541. doi: 10.1037/0278-6133.21.6.531.

Miukkolasa, B., Obminski, Z., Stupnicki, R., \& Golec, L. (1994). Effects of music treatment on salivary cortisol in patients exposed to presurgical stress. Experimental Clinical Endocrinology, 102, 118-120. doi: 10.1055/s-0029-1211273.

Morilak, D. A., Barrera, G., Echevarria, D. J., Garcia, A. S., Hernandez, A., Ma, S., \& Petre, C. O. (2005). Role of brain norepinephrine in the behavioral response to stress. Progress in Neuro-Psychopharmacology and Biological Psychiatry, 29, 1214-1224. doi: https://doi.org/10.1016/j.pnpbp.2005.08.007.

Munro, C. A., Oswald, L. M., Weerts, E. M., McCaul, M. E., \& Wand, G. S. (2005). Hormone responses to social stress in abstinent alcohol-dependent subjects and social drinkers with no history of alcohol dependence. Alcoholism: Clinical and Experimental Research, 29, 1133-1138. doi: 10.1097/01.ALC.0000172459.71517.05.

Nader, N., Chrousos, G. P., \& Kino, T. (2010). Interactions of the Circadian CLOCK system and the HPA axis. Trends in Endocrinology \& Metabolism, 21, 277-286. doi: 
10.1016/j.tem.2009.12.011.

Nater, U. M., Hoppmann, C. A., \& Scott, S. B. (2013). Diurnal profiles of salivary cortisol and alpha-

amylase change across the adult lifespan: Evidence from repeated daily life assessments. Psychoneuroendocrinology, 38, 3167-3171. doi:

http://dx.doi.org/10.1016/j.psyneuen.2013.09.008.

Nater, U. M., Abbruzzese, E., Krebs, M., \& Ehlert, U. (2006). Sex differences in emotional and psychophysical responses to musical stimuli. International Journal of Psychophysiology, 62, 300-308. doi: https://doi.org/10.1016/j.ijpsycho.2006.05.011.

Nilsson, U., Unosson, M., \& Rawal, N. (2005). Stress reduction and analgesia in patients exposed to calming music postoperatively: A randomized controlled trial. European Journal of Anaesthesiology, 22, 96-102. doi: https://doi.org/10.1017/S0265021505000189.

North, A. C., Hargreaves, D. J., \& Hargreaves, J. J. (2004). Uses of music in everyday life. Music Perception: An Interdisciplinary Journal, 22, 41-77.

Nyklicek, I., Thayer, J. F., \& Van Doornen, L. J. P. (1997). Cardiorespiratory differentiation of musically-induced emotions. Journal of Psychophysiology, 11, 304-321.

Ockenfels, M. C., Porter, L., Smyth, J., Kirschbaum, C., Hellhammer, D. H., \& Stone, A. A. (1995). Effect of chronic stress associated with unemployment on salivary cortisol: Overall cortisol levels, diurnal rhythm, and acute stress reactivity. Psychosomatic Medicine, 57, 460-467.

Park, B-J., Tsunetsugu, Y., Kasetani, T., Kagawa, T., \& Miyazaki, Y. (2010). The physiological effects of Shinrin-yoku (taking in the forest atmosphere or forest bathing): Evidence from 
field experiments in 24 forests across Japan. Environmental Health and Preventative Medicine, 15, 18-26. doi: 10.1007/s12199-009-0086-9.

Park, B-J., Tsunetsugu, Y., Kasetani, T., Morikawa, T., Kagawa, T., \& Miyazaki, Y. (2009). Physiological effects of forest recreation in a young conifer forest in Hinokage Town, Japan. Silva Fennica, 43, 291-301.

Park, B-J., Tsunetsugu, Y., Kasetani, T., Hirano, H., Kagawa, T., Sato, M., \& Miyazaki, Y. (2007). Physiological effects of Shinrin-yoku (taking in the atmosphere of the forest)using salivary cortisol and cerebral activity as indicators. Journal of Physiological Anthropology, 26, 123-128. doi: http://doi.org/10.2114/jpa2.26.123.

Park, B-J., Tsunetsugu, Y., Ishii, H., Furuhashi, S., Hirano, H., Kagawa, T., \& Miyazaki, Y. (2008). Physiological effects of Shinrin-yoku (taking in the atmosphere of the forest) in a mixed forest in Shinano Town, Japan. Scandinavian Journal of Forest Research, 23, 278283. doi: $10.1080 / 02827580802055978$.

Pascualy, M., Petrie, E. C., Brodkin, K., Peskind, E. R., Wilkinson, C. W., \& Raskind, M. A. (2000). Hypothalamic pituitary adrenocortical and sympathetic nervous system responses to the cold pressor test in Alzheimer's disease. Biological Psychiatry, 48, 247-254. doi:10.1016/S0006-3223(00)00879-9.

Petrowski, K., Herold, U., Joraschky, P., Wittchen, H-U., \& Kirschbaum, C. (2010). A striking pattern of cortisol non-responsiveness to psychosocial stress in patients with panic disorder with concurrent normal cortisol awakening responses.

Psychoneuroendocrinology, 35, 414-421. doi:10.1016/j.psyneuen.2009.08.003.

Phillips, A. C., Hunt, K., Der, G., \& Carroll, D. (2011). Blunted cardiac reactions to acute psychological stress predicts symptoms of depression five years later: Evidence from a 
large community study. Psychophysiology, 48, 142-148. doi: 10.1111/j.14698986.2010.01045.x.

Piazza, J. R., Almeida, D. M., Dmitrieva, N. O., \& Klein, L. C. (2010). Frontiers in the use of biomarkers of health in research on stress and aging. Journal of Gerontology: Psychological Sciences, 65B, 513-525. doi: 10.1093/geronb/gbq049.

Pruessner, J. C., Kirschbaum, C., Meinlschmid, G., \& Hellhammer, D. H. (2003). Two formulas for computation of area under the curve represent measures of total hormone concentration versus time-dependent change. Psychoneuroendocrinology, 28, 916-931. doi: 10.1016/S0306-4530(02)00108-7.

Pruessner, J. C., Wolf, O. T., Hellhammer, D. H., Buske-Kirschbaum, A., von Auer, S., Jobst, F.,..,Kirschbaum, C. (1997). Free cortisol levels after awakening: A reliable biological marker for the assessment of adrenocortical activity. Life Science, 61, 2539-2549. doi: https://doi.org/1016/S0024-3205(97)01008-4.

Raison, C. L., \& Miller, A. H. (2003). When not enough is too much: The role of insufficient glucocorticoid signaling in the pathophysiology of stress-related disorders. The American Journal of Psychiatry, 160, 1554-1565. doi: https://doi.org/10.1176/appi.ajp.160.9.1554.

Raskind, M. A., Peskind, E. R., \& Wilkinson, C. W. (1994). Hypothalamic-pituitary-adrenal axis regulation and human aging. Annals of the New York Academy of Sciences, 746, 327-335. doi: 10.1111/j.1749-6632.1994.tb39251.x.

Raz, N., Lindenberger, U., Rodrique, K. M., Kennedy, K. M., Head, D., Williamson, A.,...Acker, J. D. (2005). Regional brain changes in aging healthy adults: General trends, individual differences and modifiers. Cerebral Cortex, 15, 1676-1689. 
doi:10.1093/cercor/bhi044.

Remus, J. L., Stewart, L. T., Camp, R. M., Novak, C. M., \& Johnson, J. D. (2015). Interaction of metabolic stress with chronic mild stress in altering brain cytokines and sucrose preference. Behavioral Neuroscience, 129, 321-330. doi: 10.1037/bne0000056.

Rimmele, U., Seiler, R., Marti, B., Wirtz, P. H., Ehlert, U., \& Heinrichs, M. (2009). The level of physical activity affects adrenal and cardiovascular reactivity to psychosocial stress. Psychoneuroendocrinology, 34, 190-198. doi: 10.1016/j.psyneuen.2008.08.023.

Rimmele, U., Zellweger, B. C., Seiler, M. R., Mohiyeddini, C., Ehlert, U., \& Heinrichs, M. (2007). Trained men show lower cortisol, heart rate and psychological responses to psychosocial stress compared with untrained men. Psychoneuroendocrinology, 32, 627635. doi: 10.1016/j.psyneuen.2007.04.005.

Rohrmann, S., Hennig, J., \& Netter, P. (1999). Changing psychobiological stress reactions by manipulating cognitive processes. International Journal of Psychophysiology, 33, 149161. doi: 10.1016/S0167-8760(99)00036-7.

Rozanski, A., Blumenthal, J. A., \& Kaplan, J. (1999). Impact of psychological factors on the pathogenesis of cardiovascular disease and implications for therapy. Circulation, 99, 2192-2217.

Rubin, R. T., Mandell, A. J., \& Crandall, P. H. (1966). Corticosteroid responses to limbic stimulation in man: Localization of stimulus sites. Science, 153, 767-768. doi: 10.1126/science.153.3737.767.

Sandstrom, G. M., \& Russo F. A. (2010). Music hath charms: The effects of valence and arousal on recovery following an acute stressor. Music and Medicine, 2, 137-143. doi: $10.1177 / 194386210371486$. 
Salimpoor, V. N., Benovoy, M., Longo, Cooperstock, J. R., \& Zatorre, R. J. (2009). The rewarding aspects of music listening are related to degree of emotional arousal. PLoS ONE,4: e7487. doi: https://doi.org/10.1371/journal.pone.0007487.

Sandstrom, G. M., \& Russo, F. A. (2013). Absorption in music: Development of a scale to identify individuals with strong emotional responses to music. Psychology of Music, 41, 216-228. doi: 10.1177/0305735611422508.

Sapolsky, R. M., Krey, L. C., \& McEwen, B. S. (1986). The neuroendocrinology of stress and aging: The glucocorticoid hypothesis. Endocrine Reviews, 7, 284-301. doi: https://doi.org/10.1210/edrv-7-3-284.

Sapolsky, R. M., Romero, L. M., \& Munck, A. U. (2000). How do glucocorticoids influence stress responses? Integrating permissive, suppressive, stimulatory, and preparative actions. Endocrine Reviews, 21, 55-89. doi: https://doi.org/10.1210/edrv.21.1.0389.

Schommer, N. C., Hellhammer, D. H., \& Kirschbaum, C. (2003). Dissociation between reactivity of the hypothalamus-pituitary-adrenal axis and the sympathetic-adrenalmedullary system to repeated psychosocial stress. Psychosomatic Medicine, 65, 450-460. doi: 10.1097/01.PSY.0000035721.12441.17.

Schultz, P., Kirschbaum, C., Pruessner, J., \& Hellhammer, D. H. (1998). Increased free cortisol secretion after awakening in chronically stressed individuals due to work overload. Stress Medicine, 14, 91-97.

Schwabe, L., Haddad, L., \& Schachinger, H. (2008). HPA axis activation by a socially evaluated cold-pressor test. Psychoneuroendocrinology, 33, 890-895. doi:10.1016/j.psyneuen.2008.03.001.

Seeman, T. E., Singer, B., Wilkinson, C. W., \& McEwen, B. S. (2001). Gender differences in 
age-related changes in HPA axis reactivity. Psychoneuroendocrinology, 26, 225-240. doi: 10.1016/S0306-4530(00)00043-3.

Selye, H. (1936) A syndrome produced by diverse noxious agents. Nature, 138, 32-36.

Selye, H. (1976). The Stress of life ( $\left.2^{\text {nd }} e d.\right)$. New York: McGraw-Hill.

Sendelbach, S. E., Halm, M. A., Doran, K. A., Miller, E. H., \& Gaillard, P. (2006). Effects of music therapy on physiological and psychological outcomes for patients undergoing cardiac surgery. Journal of Cardiovascular Nursing, 21, 194-200.

Sephton, S. E., Sapolsky, R. M., Kraemer, H. C., \& Spiegel, D. (2000). Diurnal cortisol rhythm as a predictor of breast cancer survival. Journal of the National Cancer Institute, 92, 9941000. doi: https://doi.org/10.1093/jnci/92.12.994.

Sharpley, C. F. (2009). Neurobiological pathways between chronic stress and depression: Dysregulated adaptive mechanisms? Clinical Medicine: Psychiatry, 2, 33-45.

Sloboda, J. A., \& O’Neill, S. A. (2001). Emotions in everyday music listening. In Juslin, P. N. \& Sloboda, J. A. (Eds.). Music and Emotion: Theory and research. Series in affective science (pp. 415-429). New York, NY: Oxford University Press.

Sloboda, J. A., O’Neill, S. A., \& Ivaldi, A. (2001). Functions of music in everyday life: An exploratory study using the experience sampling method. Musicae Scientiae, 5, 9-32.

Smith, B. W., Shelley, B. M., Dalen, J., Wiggins, K., Tooley, E., \& Bernard, J. (2008). A pilot study comparing the effects of mindfulness-based and cognitive-behavioural stress reduction. The Journal of Alternative and Complementary Medicine, 14, 251-258. doi:10.1089/acm.2007.0641.

Sokhadze, E. M. (2007). Effects of music on the recovery of autonomic and electrocortical activity after stress induced by aversive visual stimuli. Applied Psychophysiological 
Biofeedback, 32, 31-50. doi: 10.1007/s10484-007-9033-y.

Song, C., Ikei, H., \& Miyazaki, Y. (2016). Physiological Effects of Nature Therapy: A Review of the Research in Japan. International Journal of Environmental Research and Public Health, 13(8), 781. http://doi.org/10.3390/ijerph13080781

Starkman, M. N., Gebarski, S. S., Berent, S., \& Schteingart, D. E. (1992). Hippocampal formation volume, memory dysfunction, and cortisol levels in patients with Cushing's syndrome. Biological Psychiatry, 32, 756-765. doi: https://doi.org/10.1016/00063233(92)90079-F.

Statistics Canada. (2013). Health fact sheets: Perceived life stress, 2013. Statistics Canada Catalogue no. 82-625-X. Ottawa. Version updated June 2014. Ottawa.

Sterling, P., \& Eyer, J. (1988). Allostasis: A new paradigm to explain arousal pathology. In Fisher, S., \& Reason, J. (Eds.), Handbook of Life Stress, Cognition, and Health. John Wiley \& Sons: New York.

Stone, A. A., Schwartz, J. E., Smyth, J. Kirschbaum, C., Cohen, S., Hellhammer, D. H., \& Grossman, S. (2001). Individual differences in the diurnal cycle of salivary free cortisol: A replication of flattened cycles for some individuals. Psychoneuroendocrinology, 26, 295-306. doi: https://doi.org/10.1016/S0306-4530(00)00057-3.

Suda, M., Morimoto, K., Obata, A., Koizumi, H., \& Maki, A. (2008). Emotional responses to music: Towards scientific perspectives on music therapy. NeuroReport, 19, 75-78. doi: 10.1097/WNR.0b013e3282f3476f.

Tarvainen, M. P., Niskanen, J-P., Lipponen, J. A., Ranta-aho, P. O., \& Karjalainen, P. A. (2014). Kubios HRV - Heart rate variability analysis software. Computer Methods and Programs in Biomedicine, 113, 210-220. doi: http://dx.doi.org/10.1016/j.cmpb.2013.07.024. 
Tellegen, A., \& Atkinson, G. (1974). Openness to absorbing and self-altering experiences ("absorption"), a trait related to hypnotic susceptibility. Journal of Abnormal Psychology, 83, 268-277. doi: 10.1037/h0036681.

Terry, P. C., Karageorghis, C. I., Mecozzi Saha, A., \& D’Auria, S. (2012). Effects of synchronous music on treadmill running among elite triatheletes. Journal of Science and Medicine in Sport, 15, 52-57. doi:http://dx.doi.org/10.1016/j.sams.2011.06.003.

Thayer, J. F., Ahs, F., Fredrikson, M., Sollers, J. J. III, \& Wager, T. D. (2012). A meta-analysis of heart rate variability and neuroimaging studies: Implications for heart rate variability as a marker of stress and health. Neuroscience and Biobehavioral Reviews, 36, 747-756. doi: 10.1016/j.neubiorev.2011.11.009.

Thoma, M. V., La Marca, R., Bronnimann, R., Finkel, L., Ehlert, U., \& Nater, U. M. (2013). The effect of music on the human stress response. PLOS One, 8, e70158. doi: 10.137/journal.pone.0070156.

Tsigos, C., \& Chrousos, G. P. (2002). Hypothalamic-pituitary-adrenal axis, neuroendocrine factors and stress. Journal of Psychosomatic Research, 53, 865-871. doi: https://doi.org/10.1016/S0022-3999(02)00429-4.

Tsuji, H., Venditti, F. J., Manders, E. S., Evans, J. C., Larson, M. G., Feldman, C. L. \& Levy, D. (1994). Reduced heart rate variability and mortality risk in an elderly cohort. The Framingham Heart Study. Circulation, 90, 878-883. doi: https://doi.org/10.1161/01.CIR.90.2.878.

Tsunetsugu, Y., Park, B-J., \& Miyazaki, Y. (2010). Trends in research related to "Shinrinyoku"(taking in the atmosphere or forest bathing) in Japan. Environmental Health and Preventative Medicine, 15, 27-37. doi: 10.1007/s12199-009-0091-z. 
Tsunetsugu, Y., Park, B-J., Ishii, H., Hirano, H., Kagawa, \& Miyazaki, Y. (2007). Physiological effect of Shinrin-yoku (taking in the atmosphere of the forest) in an old-growth broadleaf forest in Yamagata Prefecture, Japan. Journal of Physiological Anthropology, 26, 135142. doi: 10.2114/jpa2.26.135.

Tukey, J. W. (1977), Exploratory Data Analysis, Reading, MA: Addison- Wesley.

Uchino, B. N., Cacioppo, J. T., Malarkey, W. B., \& Glaser, R. (1995). Individual differences in cardiac sympathetic control predicts endocrine and immune responses to acute psychological stress. Journal of Personality and Social Psychology, 69, 736-741. doi: 10.1037/0022-3514.69.4.736.

Uchino, B. N., Birmingham, W., \& Berg, C. A. (2010). Are older adults less or more physiologically reactive? A meta-analysis of age-related differences in cardiovascular reactivity to laboratory tasks. The Journals of Gerontology, 65B, 154-162. doi: https://doi.org/10/1093/geronb/gnp127.

Uedo, N., Ishikawa, H., Morimoto, K., Ishihara, R., Narahara, H., Akedo, I.,...Fukuda, S. (2004). Reduction in salivary cortisol level by music therapy during colonoscopic examination. Hepato-gastroenterology, 51, 451-453.

Ulrich, R. S., Simons, R. F., Losito, B. D., Fiorito, E., Miles, M. A., \& Zelson, M. (1991). Stress recovery during exposure to natural and urban environments 1. Journal of Environmental Psychology, 11, 201-230. doi: https://doi.org/10.1016/S0272-4944(05)80184-7.

Ulrich-Lai, Y. M., \& Herman, J. P. (2009). Neural regulation of endocrine and autonomic stress responses. Nature Reviews Neuroscience, 10, 397-409. doi: 10.1038/nm2647.

Van Eck, M., Nicolson, N. A., \& Berkhof, J. (1998). Effects of stressful daily events on mood states: Relationships to global perceived stress. Journal of Personality and Social 
Psychology, 75, 1572-1585. doi: 10.1037/0022-3514.75.6.1572.

van Amelsvoort, L. G. P. M., Schouten, E. G., Maan, A. C., Swenne, C. A., \& Kok, F. J. (2000). Occupational determinants of heart rate variability. International Archives of Occupational and Environmental Health, 73, 255-262.

Ventura, T., Gomes, M. C., \& Carreira, T. (2012). Cortisol and anxiety response to a relaxing intervention on pregnant women awaiting amniocentesis. Psychoneuroendocrinology, 37, 148-156. doi: https://doi.org/10.1016/j.psyneuen.2011.05.016.

von Dawans, B., Kirschbaum, C., \& Heinrichs, M. (2011). The Trier Social Stress Test for groups (TSST-G): A new research tool for controlled simultaneous social stress exposure in a group format. Psychoneuroendocrinology, 36, 514-522. doi: 10.1016/j.psyneuen.2010.09.004.

Weckesser, L. J., Plessow, F., Pilhatsch, M., Muehlhan, M., Kirschbaum, C., \& Miller, R. (2014). Do venipuncture procedures induce cortisol response? A review, study, and synthesis for stress research. Psychoneuroendocrinology, 46, 88-99. doi:https://doi.org/10.1016/psyneuen.2014.04.012.

Wild, T. C., Kuiken, D., \& Schopflocher, D. (1995). The role of absorption in experiential involvement. Journal of Personality and Social Psychology, 69, 569-579. doi: 10.1037/0022-3514.69.3.569.

Yamamoto, M., Naga, S., \& Shimizu, J. (2007). Positive musical effects on two types of negative stressful conditions. Psychology of Music, 35, 249-275. doi: 10.1177/0305735607070375.

Zachariae, R., \& Jørgensen, M. M. (2000). Autonomic and psychological responses to an acute psychological stressor and relaxation: The influence of hypnotizability and absorption. International Journal of Clinical and Experimental Hypnosis, 48, 388-403. doi: 


\section{$10.1080 / 00207140008410368$.}

Zentner, M., Grandjean, D., \& Scherer, K. R. (2008). Emotions evoked by the sound of music:

Characterization, classification, and measurement. Emotion, 8, 494-521. doi:

10.1037/1528-3542.8.4.494. 